\title{
Quantum Gravity from Noncommutative Spacetime
}

\author{
Jungjai Lee* \\ Department of Physics, Daejin University, Pocheon 487-711, Korea \\ Hyun Seok Yang $\dagger^{\dagger}$ \\ School of Physics, Korea Institute for Advanced Study, Seoul 130-722, Korea
}

(Received 11 September 2014)

\begin{abstract}
We review a novel and authentic way to quantize gravity. This novel approach is based on the fact that Einstein gravity can be formulated in terms of a symplectic geometry rather than a Riemannian geometry in the context of emergent gravity. An essential step for emergent gravity is to realize the equivalence principle, the most important property in the theory of gravity (general relativity), from $U(1)$ gauge theory on a symplectic or Poisson manifold. Through the realization of the equivalence principle, which is an intrinsic property in symplectic geometry known as the Darboux theorem or the Moser lemma, one can understand how diffeomorphism symmetry arises from noncommutative $U(1)$ gauge theory; thus, gravity can emerge from the noncommutative electromagnetism, which is also an interacting theory. As a consequence, a background-independent quantum gravity in which the prior existence of any spacetime structure is not a priori assumed but is defined by using the fundamental ingredients in quantum gravity theory can be formulated. This scheme for quantum gravity can be used to resolve many notorious problems in theoretical physics, such as the cosmological constant problem, to understand the nature of dark energy, and to explain why gravity is so weak compared to other forces. In particular, it leads to a remarkable picture of what matter is. A matter field, such as leptons and quarks, simply arises as a stable localized geometry, which is a topological object in the defining algebra (noncommutative $\star$-algebra) of quantum gravity.
\end{abstract}

PACS numbers: 11.10.Nx, 02.40.Gh, 11.25.Tq

Keywords: Quantum gravity, Emergent gravity, Noncommutative field theory

*Electronic address: jjlee@daejin.ac.kr

$\dagger$ Electronic address: hsyang@kias.re.kr 


\section{INTRODUCTION}

The most important property in the theory of gravity (general relativity) is arguably the equivalence principle. The equivalence principle says that gravity can be interpreted as an inertial force, so it is always possible to locally eliminate the gravitational force by using a coordinate transformation, i.e., in a locally inertial frame. This immediately leads to a remarkable picture in which gravity has to describe a spacetime geometry rather than a force immanent in spacetime. The equivalence principle also implies that gravity is obviously a universal force such that gravity influences and is influenced by everything that carries energy. Therefore, the spacetime has to serve as a stage for everything supported on it, as well as an actor for the dynamical evolution of the stage (spacetime) itself. In order to quantize gravity, we, thus, have to cook a frying pan and a fish altogether. How?

\section{A. What Is Quantum Gravity?}

Quantum gravity means to "quantize" gravity. Gravity, according to Einstein's general relativity, is the dynamics of spacetime geometry where spacetime is a Riemannian manifold and the gravitational field is represented by a Riemannian metric. Thus, naively quantum gravity is meant to quantize the Riemannian manifold. However, how to "quantize" a Riemannian manifold is still vague.

Quantum mechanics has constituted a prominent example of "quantization" since its foundation. However, quantization in this case is controlled by the Planck constant $\hbar$, whose physical dimension is length times momentum, i.e., $(x \times p)$. Therefore, quantization in terms of $\hbar$ quantizes (or deforms in a weak sense) a particle phase space, as we know very well. Because we consider a classical field $\phi(x) \in C^{\infty}(M)$ to be a smooth function defined on a spacetime $M$ and a many-body system describing infinitely many particles distributed over the spacetime $M$, quantum field theory is also defined by quantization in terms of $\hbar$ in an infinite-dimensional particle phase space, as we clearly know.

Now consider "quantizing" gravity. With $\hbar$ again? Because gravity is characterized by its own intrinsic scale given by the Newton constant $G$, where the classical gravity corresponds to the $G \rightarrow 0$ limit [1], we should leave open the possibility that the quantum gravity is defined by a deformation in terms of $G$ instead of $\hbar$. Customarily, we have taken the same route to the 
quantization of gravity as that taken by conventional quantum field theory. Thus, conventional quantum gravity also intends to quantize an infinite-dimensional particle phase space associated with the metric field $g_{\mu \nu}(x)$ (or its variants such as the Ashtekar variables or spin networks) of a Riemannian geometry. However, we have to carefully contemplate whether our routine approach to quantum gravity is on the right track or not because gravity is very different from other forces, such as the electromagnetic, weak and strong forces. For a delightful journey to quantum gravity, it is necessary to pin down the precise object of quantization ( $\hbar$ or $G$ ) and clearly specify correct variables for quantization (spacetime itself or fields associated with the spacetime). For these reasons, we pose the following two questions [2]:

\section{Q1. Is gravity fundamental or emergent?}

\section{Q2. Which quantization defines quantum gravity: $\hbar$ or $G$ ?}

In Q1, we usually refer to a physical entity (force or field) as being fundamental when it does not have any superordinate substructure, and emergence usually means the arising of novel and coherent structures, patterns and properties through the collective interactions of more fundamental entities: for example, the superconductivity in condensed matter system or the organization of life in biology. Regarding to question Q1, it is quite amazing to notice that the picture of emergent gravity was already incoded in Cartan's formulation of gravity [3]. In general relativity, the gravitational force is represented by a Riemannian metric of the curved spacetime manifold $M$ :

$$
\left(\frac{\partial}{\partial s}\right)^{2}=g^{\mu \nu}(y) \frac{\partial}{\partial y^{\mu}} \otimes \frac{\partial}{\partial y^{\nu}}
$$

Cartan showed that the metric in Eq. (1) could be defined by the tensor product of two vector fields $E_{a}=E_{a}^{\mu}(y) \frac{\partial}{\partial y^{\mu}} \in \Gamma(T M)$ as follows:

$$
\left(\frac{\partial}{\partial s}\right)^{2}=\eta^{a b} E_{a} \otimes E_{b}
$$

Mathematically, a vector field $X$ on a smooth manifold $M$ is a derivation of the algebra $C^{\infty}(M)$. Here, the vector fields $E_{a} \in \Gamma(T M)$ are smooth sections of the tangent bundle $T M \rightarrow M$ which are dual to the covectors $E^{a}=E_{\mu}^{a}(y) d y^{\mu} \in \Gamma\left(T^{*} M\right)$; i.e., $\left\langle E^{b}, E_{a}\right\rangle=\delta_{a}^{b}$. The expression in Eq. (2) glimpses the avatar of gravity that a spin-two graviton might arise as a composite of two spinone vector fields. In other words, Eq. (2) can be abstracted by using the relation $(1 \otimes 1)_{S}=2 \oplus 0$. Incidentally, both mathematician and physicist use the same word, vector field, in spite of a bit different meaning.

Equation (2) suggests that we need gauge fields that take the values in the Lie algebra of diffeomorphisms in order to realize a composite graviton from spin-one vector fields. To be precise, 
the vector fields $E_{a}=E_{a}^{\mu}(y) \frac{\partial}{\partial y^{\mu}} \in \Gamma(T M)$ will be identified with "0-dimensional" gauge fields satisfying the Lie algebra

$$
\left[E_{a}, E_{b}\right]=-f_{a b}^{c} E_{c}
$$

Of course, the Standard Model does not have such kind of gauge fields, but we will see later that the desired vector fields arise from the electromagnetic fields living in noncommutative spacetime [47]. Thus, the noncommutative spacetime will allow a novel unification between electromagnetism and Einstein gravity in a completely different context from the Kaluza-Klein unification.

Regarding question Q2, we are willing to ponder the possibility that the Newton constant $G$ signifies an intrinsic Poisson structure $\theta=\frac{1}{2} \theta^{\mu \nu}(y) \frac{\partial}{\partial y^{\mu}} \wedge \frac{\partial}{\partial y^{\nu}} \in \Gamma\left(\Lambda^{2} T M\right)$ of spacetime because the gravitational constant $G$ carries the physical dimension of (length) ${ }^{2}$ in natural units. Recall that the particle phase space $P$ has its intrinsic Poisson structure $\eta=\hbar \frac{\partial}{\partial x^{i}} \wedge \frac{\partial}{\partial p_{i}}$ [8] so that the Poisson bracket on the vector space $C^{\infty}(P)$ is given by

$$
\{f, g\}_{\hbar}(x, p)=\hbar\left(\frac{\partial f}{\partial x^{i}} \frac{\partial g}{\partial p_{i}}-\frac{\partial f}{\partial p_{i}} \frac{\partial g}{\partial x^{i}}\right)
$$

where $f, g \in C^{\infty}(P)$. Here, we have intentionally inserted the Planck constant $\hbar$ into $\eta$ to make it dimensionless like $\theta$. Then, the canonical quantization can be done by association with a commutative algebra $C^{\infty}(P)$ of physical observables of a classical mechanical system, a noncommutative algebra $\mathcal{A}_{\hbar}$ of linear operators on a suitable Hilbert space $\mathcal{H}$. That is, the physical observables $f, g \in C^{\infty}(P)$ are replaced by self-adjoint operators $\widehat{f}, \widehat{g} \in \mathcal{A}_{\hbar}$ acting on $\mathcal{H}$, and the Poisson bracket in Eq. (4) is replaced by a quantum bracket

$$
\{f, g\}_{\hbar} \rightarrow-i[\widehat{f}, \widehat{g}]
$$

This completes the quantization of the mechanics of a particle system whose phase space $P$ is now noncommutative.

In the same way, one can define a Poisson bracket $\{\cdot, \cdot\}_{\theta}: C^{\infty}(M) \times C^{\infty}(M) \rightarrow C^{\infty}(M)$ by using the Poisson structure $\theta \in \Gamma\left(\Lambda^{2} T M\right)$ of the spacetime manifold $M$ :

$$
\{f, g\}_{\theta}(y)=\theta(d f, d g)=\theta^{\mu \nu}(y)\left(\frac{\partial f}{\partial y^{\mu}} \frac{\partial g}{\partial y^{\nu}}-\frac{\partial f}{\partial y^{\nu}} \frac{\partial g}{\partial y^{\mu}}\right)
$$

where $f, g \in C^{\infty}(M)$. In the case where $\theta^{\mu \nu}$ is a constant cosymplectic matrix of rank $2 n$, one can apply the same canonical quantization to the Poisson manifold $(M, \theta)$. One can associate to a commutative algebra $\left(C^{\infty}(M),\{\cdot, \cdot\}_{\theta}\right)$ of smooth functions defined on the spacetime $M$, a 
noncommutative algebra $\mathcal{A}_{\theta}$ of linear operators on a suitable Hilbert space $\mathcal{H}$. That is, the smooth functions $f, g \in C^{\infty}(M)$ become noncommutative operators (fields) $\widehat{f}, \widehat{g} \in \mathcal{A}_{\theta}$ acting on $\mathcal{H}$, and the Poisson bracket in Eq. (6) is replaced by a noncommutative bracket [9, 10]

$$
\{f, g\}_{\theta} \rightarrow-i[\widehat{f}, \widehat{g}]
$$

This completes the quantization of the Poisson algebra $\left(C^{\infty}(M),\{\cdot, \cdot\}_{\theta}\right)$ where spacetime $M$ is now noncommutative, i.e.,

$$
\left[y^{\mu}, y^{\nu}\right]=i \theta^{\mu \nu}
$$

Throughout the paper, we will omit the hat for noncommutative coordinates $y^{\mu} \in \mathcal{A}_{\theta}$ for notational convenience.

The question still remains. What is the relation between the Poisson algebra $\left(C^{\infty}(M),\{\cdot, \cdot\}_{\theta}\right)$ and (quantum) gravity? We will not try to answer the question right now. Instead, we want to point out that the vector fields in Eq. (2) can be derived from the Poisson algebra [4-7]. It is well-known [8] that, for a given Poisson algebra $\left(C^{\infty}(M),\{\cdot, \cdot\}_{\theta}\right)$, there exists a natural map $C^{\infty}(M) \rightarrow T M: f \mapsto X_{f}$ between smooth functions in $C^{\infty}(M)$ and vector fields in $T M$ such that

$$
X_{f}(g)=\{f, g\}_{\theta}
$$

for any $g \in C^{\infty}(M)$. Indeed, the assignment in Eq. (9) between a Hamiltonian function $f$ and the corresponding Hamiltonian vector field $X_{f}$ is the Lie algebra homomorphism in the sense

$$
X_{\{f, g\}_{\theta}}=\left[X_{f}, X_{g}\right]
$$

where the right-hand side represents the Lie bracket between the Hamiltonian vector fields.

Motivated by the homomorphism between the Poisson algebra $\left(C^{\infty}(M),\{\cdot, \cdot\}_{\theta}\right)$ and the Lie algebra of vector fields [8], one may venture to formulate Einstein gravity in terms of a symplectic or a Poisson geometry rather than a Riemannian geometry. Suppose that there is a set of fields defined on a symplectic manifold $M$

$$
\left\{D_{a}(y) \in C^{\infty}(M) \mid y \in M, a=1, \cdots, 2 n\right\}
$$

According to the map in Eq. (9), the smooth functions in Eq. (11) can be mapped to vector fields as follows:

$$
V_{a}[f](y) \equiv\left\{D_{a}(y), f(y)\right\}_{\theta}=-\theta^{\mu \nu} \frac{\partial D_{a}(y)}{\partial y^{\nu}} \frac{\partial f(y)}{\partial y^{\mu}} .
$$


The vector fields $V_{a}(y)=V_{a}^{\mu}(y) \frac{\partial}{\partial y^{\mu}} \in \Gamma(T M)$ take values in the Lie algebra of volume-preserving diffeomorphisms because $\partial_{\mu} V_{a}^{\mu}=0$ by definition. Because the vector fields $V_{a}$ need not be orthonormal though they are orthogonal frames, it will be possible to relate the vector fields $V_{a} \in$ $\Gamma(T M)$ to the orthonormal frames (vielbeins) $E_{a}$ in Eq. (2) by $V_{a}=\lambda E_{a}$, with $\lambda \in C^{\infty}(M)$ to be determined [7].

Our above reasoning implies that a field theory equipped with the fields in Eq. (11) on a symplectic or a Poisson manifold may give rise to Einstein gravity. If this is the case, quantum gravity will be much more accessible because there is a natural symplectic or Poisson structure, so it is obvious how to quantize the underlying system, as was already done in Eqs. (77) and (8). Following this line of thought, we will aim to answer the question what quantum gravity is by carefully addressing the issues in Q1 and Q2.

\section{B. Quartet of Physical Constants}

The physical constants defining a theory prescribe all the essential properties of the theory. Planck realized that the union of three "fundamental" constants in Nature, the Planck constant $\hbar$ in quantum mechanics, the universal velocity $c$ in relativity, and the Newton constant $G$ in gravity, uniquely fixed the characteristic scales for quantum gravity:

$$
\begin{aligned}
& M_{p l}=\sqrt{\frac{c \hbar}{G}}=2.2 \times 10^{-5} \mathrm{~g}, \\
& L_{p l}=\sqrt{\frac{G \hbar}{c^{3}}}=1.6 \times 10^{-33} \mathrm{~cm}, \\
& T_{p l}=\sqrt{\frac{G \hbar}{c^{5}}}=5.4 \times 10^{-44} \mathrm{~s} .
\end{aligned}
$$

The expression in Eq. (13) holds in four dimensions, and it has a different expression in other dimensions. Interestingly, one cannot construct a dimensionless quantity out of the three constants except in two dimensions. In two dimensions, the combination $G \hbar / c^{3}$ is a dimensionless quantity. This may be understood by noticing that pure two-dimensional gravity is topological, so it should not trigger any dynamical scale. Therefore, except for two dimensions, each constant must play an independent role in the theory of quantum gravity.

Recent developments in theoretical physics have revealed in many ways that gravity may not be a fundamental, but rather an emergent, phenomenon, as string theory has demonstrated in the last decade [11]. This means that the Newton constant $G$ can be determined by using some of 
the quantities in a theory defining emergent gravity. Because we want to derive gravity from some gauge theory, it is proper to consider the quartet of physical constants by adding a coupling constant $e$, which is the electric charge. A general gauge coupling constant sometimes will be denoted by $g_{Y M}$. Using the symbol $L$ for length, $T$ for time, and $M$ for mass and writing $[X]$ for the dimension of some physical quantity $X$, we have the following in $D$ dimensions:

$$
\begin{aligned}
& {[\hbar]=M L^{2} T^{-1},} \\
& {[c]=L T^{-1},} \\
& {\left[e^{2}\right]=M L^{D-1} T^{-2},} \\
& {[G]=M^{-1} L^{D-1} T^{-2} .}
\end{aligned}
$$

A remarkable point of the system in Eq. (14) is that it specifies the following intrinsic scales independently of dimensions:

$$
\begin{aligned}
& M^{2}=\left[\frac{e^{2}}{G}\right], \\
& L^{2}=\left[\frac{G \hbar^{2}}{e^{2} c^{2}}\right], \\
& T^{2}=\left[\frac{G \hbar^{2}}{e^{2} c^{4}}\right] .
\end{aligned}
$$

From the four-dimensional case where $e^{2} / \hbar c \approx 1 / 137$, one can see that the scales in Eq. (15) are not so different from the Planck scales in Eq. (13).

Note that the first relation $G M^{2}=e^{2}$ in Eq. (15) implies that at the mass scale $M$, the gravitational and the electromagnetic interactions become of equal strength. This is a desirable property for our purpose because we want to derive gravity from gauge theory! Also, the system in Eq. (14) should be expected to admit a dimensionless quantity because the four quantities are determined by three variables. That quantity is given by the following combination:

$$
\left(\frac{e c}{\sqrt{G} \hbar}\right)^{D} \cdot \frac{\hbar^{3} G^{2}}{c^{5} e^{2}}=\text { dimensionless. }
$$

One can see from Eq. (16) that in lower dimensions, it is possible to construct a dimensionless quantity out of only three parameters: $\{\hbar, c, G\}$ in $D=2,\{c, e, G\}$ in $D=3,\{\hbar, c, e\}$ in $D=4$, and $\{\hbar, e, G\}$ in $D=5$. In $D \geq 6$ dimensions, we need all of the four constants in Eq. (14) to have a dimensionless quantity. This smells of interesting hidden physics, but we have not yet figured out what it is [12]. However, we will clearly see what the scales in Eq. (15) set by the system in Eq. (14) mean. Notice that the length $L$ in Eq. (15) is the Compton wavelength of mass $M$ for 
which the gravitational and the electromagnetic interactions have the same strength. It turns out to be the scale of spacetime noncommutativity where the conspiracy between gravity and gauge theory takes place.

Equation (15) implies that, if gauge theory whose coupling constant is given by $e$ is equipped with an intrinsic length scale $L$, the Newton constant $G$ can be determined by using field theory parameters only, i.e., $\frac{G \hbar^{2}}{c^{2}} \sim e^{2} L^{2}$, hinting an intimate correspondence between gravity and gauge theory [7]. For example, a noncommutative gauge theory and a field theory on a D-brane are cases where $L^{2}$ can be identified with $|\theta|$, the noncommutativity of spacetime, for the former and with $2 \pi \alpha^{\prime}$, the size of a string, for the latter [13]. As we will discuss later, a theory of quantum gravity must be background independent; thus, the dynamical scale for quantum gravity will be generated by a vacuum condensate that is exactly one in Eq. (15).

\section{Noncommutative Spacetime as Quantum Geometry}

We have discussed some reasons the gravitational constant $G$ dictates a symplectic or a Poisson structure to spacetime $M$. Thereby, a field theory will be defined on a symplectic manifold, and, as we argued before, Einstein gravity may arise from the field theory. If this is the case, quantum gravity will be defined by quantizing the field theory in terms of the underlying Poisson structure, which is simply a Dirac quantization such as Eq. (7) for a canonical symplectic structure. Therefore, if all these are smoothly working, quantum geometry can be defined by using a field theory on a noncommutative space such as Eq. (8). Let us briefly sketch how that is possible.

A symplectic structure $B=\frac{1}{2} B_{\mu \nu} d y^{\mu} \wedge d y^{\nu}$ of spacetime $M$ defines a Poisson structure $\theta^{\mu \nu} \equiv$ $\left(B^{-1}\right)^{\mu \nu}$ on $M$, where $\mu, \nu=1, \ldots, 2 n$. The Dirac quantization with respect to the Poisson structure $\theta^{\mu \nu}$ then leads to the quantum phase space in Eq. (8). Because the Poisson structure $\theta^{\mu \nu}$ defines canonical pairs between noncommutative coordinates $y^{\mu} \in \mathcal{A}_{\theta}$, one can introduce annihilation and creation operators, $a_{i}$ and $a_{j}^{\dagger}, i, j=1, \cdots, n$, by using those pairs. For example, $a_{1}=\left(y^{1}+i y^{2}\right) / \sqrt{2 \theta^{12}}, a_{1}^{\dagger}=\left(y^{1}-i y^{2}\right) / \sqrt{2 \theta^{12}}$, etc. Then, the Moyal algebra in Eq. (8) is equal to the Heisenberg algebra of an $n$-dimensional harmonic oscillator, i.e.,

$$
\left[y^{\mu}, y^{\nu}\right]=i \theta^{\mu \nu} \Leftrightarrow\left[a_{i}, a_{j}^{\dagger}\right]=\delta_{i j}
$$

From quantum mechanics, the representation space of noncommutative $\mathbb{R}^{2 n}$ is well-known to 
be given by an infinite-dimensional, separable Hilbert space

$$
\mathcal{H}=\left\{|\vec{n}\rangle \equiv\left|n_{1}, \cdots, n_{n}\right\rangle, n_{i}=0,1, \cdots\right\}
$$

that is orthonormal, i.e., $\langle\vec{n} \mid \vec{m}\rangle=\delta_{\vec{n} \vec{m}}$, and complete, i.e., $\sum_{\vec{n}=0}^{\infty}|\vec{n}\rangle\langle\vec{n}|=1$. Note that any smooth function on a noncommutative space can be represented by an operator acting on an appropriate Hilbert space $\mathcal{H}$, which consists of a noncommutative $\star$-algebra $\mathcal{A}_{\theta}$, like a set of observables in quantum mechanics. Therefore, any field $\widehat{\Phi} \in \mathcal{A}_{\theta}$ in the noncommutative space in Eq. (17) becomes an operator acting on the Fock space $\mathcal{H}$ and can be expanded in terms of the complete operator basis

$$
\mathcal{A}_{\theta}=\left\{|\vec{n}\rangle\langle\vec{m}|, n_{i}, m_{j}=0,1, \cdots\right\}
$$

that is,

$$
\widehat{\Phi}(y)=\sum_{\vec{n}, \vec{m}} \Phi_{\vec{n} \vec{m}}|\vec{n}\rangle\langle\vec{m}|
$$

One may use the 'Cantor diagonal method' to put the $n$-dimensional non-negative integer lattice in $\mathcal{H}$ into a one-to-one correspondence with the infinite set of natural numbers (i.e., 1-dimensional positive integer lattice): $|\vec{n}\rangle \leftrightarrow|n\rangle, n=1, \cdots, N \rightarrow \infty$. In this one-dimensional basis, Eq. (20) can be relabeled in the following form:

$$
\widehat{\Phi}(y)=\sum_{n, m=1}^{\infty} M_{n m}|n\rangle\langle m| .
$$

One can regard $M_{n m}$ in Eq. (21) as elements of an $N \times N$ matrix $M$ in the $N \rightarrow \infty$ limit. We then get the following important relation [9, 10]:

$$
\text { Any field on noncommutative } \mathbb{R}^{2 n} \cong N \times N \text { matrix at } N \rightarrow \infty \text {. }
$$

If the field $\Phi(y) \in C^{\infty}(M)$ was originally a real field, then $M$ should be a Hermitian matrix. The relation in Eq. (22) means that a field in the noncommutative $\star$-algebra $\mathcal{A}_{\theta}$ can be regarded as a master field of a large $N$ matrix.

Now the very notion of points in a noncommutative space, such as the Moyal space in Eq. (8), is doomed; instead the points are replaced by states in $\mathcal{H}$. Thus, the usual concept of geometry based on a smooth manifold will be replaced by a theory of operator algebra, e.g., the noncommutative geometry à la Connes [14] or the theory of deformation quantization à la Kontsevich 
[15]. Furthermore, through the matrix representation in Eq. (22) of the operator algebra, one can achieve a coordinate-free description of quantum field theory. Therefore, it should be possible to achieve a background-independent formulation of quantum gravity in which the interactions between fundamental ingredients can be defined without introducing any spacetime structure [7]. The most natural objects for that purpose are algebra-valued fields, which can be identified with elements in a noncommutative $\star$-algebra such as noncommutative gauge fields [16].

Several matrix models [17-19] have appeared in string theory. They have illustrated how the matrix model can be regarded as a nonperturbative formulation of gravity or string theory in the sense that it describes a quantized geometry with an arbitrary topology. Matrix theory contains multiple branes with arbitrary topologies as its spectrum and allows a topology change of the spacetime manifold as a sequence of the change of matrix data [20].

\section{Outline of the Paper}

In this review, we will not survey other approaches to emergent gravity because good expositions [21] already exist and our approach is quite different, although the underlying philosophy may be the same. Our unique clue is based on the fact that Einstein gravity can be formulated in terms of a symplectic geometry [7]. Basically, we are considering a symplectic geometry as a commutative limit of a noncommutative geometry that is regarded as a microscopic structure of spacetime, just as classical mechanics on a mundane scale is simply a coarse graining of quantum mechanics in the atomic world. A Riemannian geometry, thus, appears in a macroscopic world as a coarse graining of the noncommutative geometry, as we already briefly outlined.

Our line of thought has been motivated by several similar ideas, mostly by the AdS/CFT correspondence [22] and matrix models [17-19] in string theory. Also, the work by Rivelles [23] and the following works [24] triggered thoughts about a noncommutative field theory as a theory of gravity. A series of interesting works along this line has recently been conducted by Steinacker and his collaborators [25-27]. See his recent review [28] and references therein. Also, there are many closely related works [29-37]. However, the emergent gravity based on noncommutative field theories is relatively new, so it would be premature to have an extensive review about this subject because it is still in an early stage of development. Therefore we will focus on ours. Although this review is basically a coherent survey of recent works [4-7, 38-42] of the second author, it also contains several new results and many clarifications, together with important pictures about 
quantum gravity. Early reviews can be found in Refs. [39] and [40].

In Section II we elucidate the reason Einstein gravity can emerge from electromagnetism as long as spacetime admits a symplectic structure and explicitly show how Einstein's equations can be derived from the equations of motion for electromagnetic fields on a symplectic spacetime. The emergent gravity we propose here actually corresponds to a field theory realization of open-closed string duality or large- $N$ duality in string theory and to a generalization of homological mirror symmetry [43] in the sense that the deformation of the symplectic structure $\omega$ is isomorphic to the deformation of the Riemannian metric $g$ in the triple $(M, g, \omega)$. In Section II.A, we explain the equivalence principle for the electromagnetic force, stating that there always exists a coordinate transformation to locally eliminate the electromagnetic force if spacetime supports a symplectic structure. This important property for emergent gravity originates from the Darboux theorem [44] or the Moser lemma [45] in symplectic geometry, which also explains how diffeomorphism symmetry in general relativity arises from such a gauge theory. We explain how the equations of motion for $U(1)$ gauge fields are mapped to those for vector fields defined by Eq. (12). In Section II.B, we first initiate the emergent gravity by showing [4] that self-dual electromagnetism on a symplectic 4-manifold is equivalent to self-dual Einstein gravity. Although it was previously proved in Ref. [7], we newly prove it again in a more geometric way to illustrate how elegant and beautiful the emergent gravity is. In Section II.C, we derive Einstein's equations from the electromagnetism on a symplectic manifold, rigorously confirming the speculation in Section I.A. We find [7] that the emergent gravity from electromagnetism predicts some exotic energy whose physical nature will be identified in Section V.B.

In Section III, we discuss how to quantize Einstein gravity in the context of emergent gravity by using the canonical quantization in Eq. (7) of a spacetime Poisson manifold. We argue in Section III.A that the equivalence principle for the emergent gravity can be lifted to a noncommutative spacetime such as Eq. (8). The equivalence principle in a noncommutative spacetime is realized as a gauge equivalence between star products in the context of deformation quantization [15], and so dubbed the "quantum equivalence principle" [5]. This implies that quantum gravity can consistently be derived from the quantum equivalence principle and that matter fields can arise from the quantized spacetime. In Section III.B, it is shown [6] that the emergent gravity from a noncommutative $\star$-algebra $\mathcal{A}_{\theta}$ can be understood as a large- $N$ duality such as the AdS/CFT correspondence and the matrix models in string theory. The gravitational metric determined by large- $N$ matrices or noncommutative gauge fields is explicitly derived. We clarify in Section III.C how emergent 
gravity achieves the background-independent formulation in which any kind of spacetime structure is not a priori assumed, but is defined by the theory. An important picture of emergent gravity is identified. If a classical geometry is to be described from a background-independent theory, it is necessary to have a nontrivial vacuum defined by a coherent condensation of gauge fields, e.g., the vacuum defined by Eq. (17), which is also the origin of a spacetime symplectic or a Poisson structure such as Eq. (6). In Section III.D, the emergent gravity is generalized to a generic noncommutative spacetime such as the case with $\theta^{\mu \nu}=$ nonconstant [5] and a general Poisson manifold [42].

In Section IV, we speculate what particles and matter fields are and how they arise from a noncommutative $\star$-algebra $\mathcal{A}_{\theta}$. We claim that a matter field, such as quarks and leptons, is defined by a stable localized geometry, which is a topological object in the defining algebra (noncommutative $\star$-algebra) of quantum gravity [7]. First we review in Section IV.A Feynman's view [46-48] of the electrodynamics of a charged particle to understand why an extra internal space is necessary to introduce the weak and the strong forces. The extra dimensions appear with a Poisson structure of Lie algebra type implemented with some localizability condition to stabilize the internal space. In Section IV.B, we understand the Feynman's derivation of gauge forces as the Darboux transformation in Eq. (29) between two symplectic structures where one of them is a deformation of the other in terms of external gauge fields. This beautiful idea is not ours, but was noticed at Ref. [49] long ago. In Section IV.C, we define a stable state in a large- $N$ gauge theory and relate it to the K-theory [50-53]. With the correspondence in Eq. (22), the K-theory class is mapped to the K-theory of a noncommutative $\star$-algebra $\mathcal{A}_{\theta}$. We argue, using the well-known, but rather mysterious, math, the Atiyah-Bott-Shapiro construction of K-theory generators in terms of the Clifford module [54], that the topological object defined by large- $N$ matrices or the noncommutative $\star$-algebra $\mathcal{A}_{\theta}$ describes fermions such as quarks and leptons. It turns out that an extra noncommutative space is essential to realize the weak and the strong forces.

In Section V, we discuss the most beautiful aspects of emergent gravity. Remarkably, emergent gravity reveals a novel picture about the origin of spacetime, dubbed as emergent spacetime, which is radically different from any previous physical theories, all of which describe what happens in a given spacetime. In Section V.A, we point out that the concept of emergent time is naturally defined as long as spacetime admits an intrinsic symplectic or Poisson structure. The time evolution of a spacetime geometry is defined by Hamilton's equation defined by the spacetime Poisson structure, Eq. (6). Because the symplectic structure triggered by the vacuum condensate in Eq. 
(17) not only causes the emergence of spacetime but also specifies an orientation of spacetime manifold, we are tempted to conceive that the emergent gravity may explain the arrow of time in the cosmic evolution of our Universe - the most notoriously difficult problem in quantum gravity. We analyze in Section V.B the anatomy of spacetime derived from a noncommutative gauge theory or large- $N$ matrix model. We explain why there is no cosmological constant problem in emergent gravity [41]. We point out that a vacuum energy of a Planck scale does not gravitate, unlike Einstein gravity and that a flat spacetime is emergent from the Planck energy condensation in vacuum. Finally, we try to identify the physical nature of the exotic energy-momentum tensor whose existence was predicted in Section II.C. Surprisingly, it mimics all the properties of dark energy, so we suggest the energy as a plausible candidate for dark energy [7]. If so, the quantum gravity defined by noncommutative gauge theory seems to resolve many notorious problems in theoretical physics: for example, the cosmological constant problem, the nature of dark energy and the reason gravity is so weak compared to other forces.

In the final section, we try to understand why the emergent gravity defined by a noncommutative geometry resembles string theory. We conclude with several remarks about important open issues and speculate on a proper mathematical framework for emergent gravity and quantum gravity.

\section{EMERGENT GRAVITY}

In order to argue that gravity can emerge from some field theory, it is important to identify how the essential properties of gravity can be realized in the underlying field theory. If not, the emergent gravity cannot physically be viable. Therefore, we will reasonably argue how the equivalence

principle, the most important property in the theory of gravity (general relativity), can be realized from $U(1)$ gauge theory on a symplectic manifold $M$. Through the realization of the equivalence principle in the context of symplectic geometry, we can understand how diffeomorphism symmetry arises from noncommutative $U(1)$ gauge theory and gravity can emerge from noncommutative electromagnetism, which is also an interacting theory.

\section{A. The Equivalence Principle from Symplectic Geometry}

Consider a $U(1)$ bundle supported on a symplectic manifold $(M, B)$. Physically, we are considering open strings moving on a D-brane whose data are given by $(M, g, B)$, where $M$ is a smooth 
manifold equipped with a metric $g$ and a symplectic structure $B$. The worldsheet action of open strings, with a compact notation, reads as

$$
S=\frac{1}{4 \pi \alpha^{\prime}} \int_{\Sigma}|d X|^{2}-\int_{\Sigma} B-\int_{\partial \Sigma} A,
$$

where $X: \Sigma \rightarrow M$ is a map from an open string worldsheet $\Sigma$ to a target spacetime $M$ and $B(\Sigma)=X^{*} B(M)$ and $A(\partial \Sigma)=X^{*} A(M)$ are pull-backs of spacetime gauge fields to the worldsheet $\Sigma$ and the worldsheet boundary $\partial \Sigma$, respectively.

From the compact notation in Eq. (23), it is obvious that the string action in Eq. (23) respects the following local gauge symmetries:

(I) $\operatorname{Diff}(M)$-symmetry

$$
X \rightarrow X^{\prime}=X^{\prime}(X) \in \operatorname{Diff}(M)
$$

(II) $\Lambda$-symmetry

$$
(B, A) \rightarrow(B-d \Lambda, A+\Lambda)
$$

where the gauge parameter $\Lambda$ is a one-form in $M$. Note that the $\Lambda$-symmetry is present only when $B \neq 0$, so it is a stringy symmetry by nature. When $B=0$, the symmetry in Eq. (25) is reduced to $A \rightarrow A+d \lambda$, which is the ordinary $U(1)$ gauge symmetry because $A$ is a connection of the $U(1)$ bundle.

The $\Lambda$-symmetry then predicts a very important result. The presence of a $U(1)$ bundle on a symplectic manifold $(M, B)$ should appear only with the combination $\Omega=B+F$, where $F=d A$ because $\Omega$ is the only gauge-invariant 2 -form under the transformation in Eq. (25). Because we regard $B \in \Gamma\left(\Lambda^{2} T^{*} M\right)$ as a symplectic structure over $M$, the electromagnetic force $F=d A$ appears only as the local deformation of the symplectic structure $\Omega(x)=(B+F)(x)$.

Another important result derived from the open string action, Eq. (23), is that the triple $(M, g, B)$ comes only in the combination $(M, g+\kappa B)$, where $\kappa \equiv 2 \pi \alpha^{\prime}=2 \pi l_{s}^{2}$ denotes the string scale. Note that the Riemannian metric $g$ and the symplectic structure $B$ in the triple $(M, g, B)$ can be regarded as an bundle isomorphism from a tangent bundle $T M$ to a cotangent bundle $T^{*} M$ because both are nondegenerate bilinear maps on $T M$, i.e., $(g, B): T M \rightarrow T^{*} M$. Therefore, the socalled DBI "metric" $g+\kappa B: T M \rightarrow T^{*} M$, which maps $X \in T M$ to $\xi=(g+\kappa B)(X) \in T^{*} M$, embodies a generalized geometry [55] continuously interpolating between a symplectic geometry $\left(\left|\kappa B g^{-1}\right| \gg 1\right)$ and a Riemannian geometry $\left(\left|\kappa B g^{-1}\right| \ll 1\right)$. Including the excitation of open 
strings, one can combine the two results to conclude that the data of ' $\mathrm{D}$-manifold' are given by $(M, g, \Omega)=(M, g+\kappa \Omega)$.

Consider another D-brane whose 'D-manifold' is described by different data $(N, G, B)=$ $(N, G+\kappa B)$. A question is whether there exists a diffeomorphism $\phi: N \rightarrow M$ such that $\phi^{*}(g+\kappa \Omega)=G+\kappa B$ on $N$. In order to answer the question, let us shortly digress to some important aspects of simplectic geometry. The symplectic geometry respects an important property, known as the Darboux theorem [44], stating that every symplectic manifold of the same dimension is locally indistinguishable [8]. To be precise, let $(M, \omega)$ be a symplectic manifold. Consider a smooth family $\omega_{t}=\omega_{0}+t\left(\omega_{1}-\omega_{0}\right)$ of symplectic forms joining $\omega_{0}$ to $\omega_{1}$, where $\left[\omega_{0}\right]=\left[\omega_{1}\right] \in H^{2}(M)$ and $\omega_{t}$ is symplectic $\forall t \in[0,1]$. A remarkable point (due to the Moser lemma [45]) is that there exists a one-parameter family of diffeomorphisms $\phi: M \times \mathbb{R} \rightarrow M$ such that $\phi_{t}^{*}\left(\omega_{t}\right)=\omega_{0}, 0 \leq t \leq 1$. If there exist such diffeomorphisms as a flow generated by time-dependent vector fields $X_{t} \equiv \frac{d \phi_{t}}{d t} \circ \phi_{t}^{-1}$, one would have for all $0 \leq t \leq 1$ that

$$
\mathcal{L}_{X_{t}} \omega_{t}+\frac{d \omega_{t}}{d t}=0
$$

because, by the Lie derivative formula, one has

$$
\begin{aligned}
0=\frac{d}{d t}\left(\phi_{t}^{*} \omega_{t}\right) & =\phi_{t}^{*}\left(\mathcal{L}_{X_{t}} \omega_{t}\right)+\phi_{t}^{*} \frac{d \omega_{t}}{d t} \\
& =\phi_{t}^{*}\left(\mathcal{L}_{X_{t}} \omega_{t}+\frac{d \omega_{t}}{d t}\right) .
\end{aligned}
$$

Using Cartan's magic formula, $\mathcal{L}_{X}=d \iota_{X}+\iota_{X} d$, for the Lie derivative along the flow of a vector field $X$, one can see that Eq. (26) can be reduced to Moser's equation

$$
\iota_{X} \omega_{t}+A=0
$$

where $\omega_{1}-\omega_{0}=d A$.

In summary, there always exists a one-parameter family of diffeomorphisms as a flow generated by a smooth family of time-dependent vector fields $X_{t}$ satisfying Eq. (28) for the change of the symplectic structure within the same cohomology class from $\omega_{0}$ to $\omega_{1}$, where $\omega_{1}-\omega_{0}=d A$. The evolution of the symplectic structure is locally described by the flow $\phi_{t}$ of $X_{t}$ satisfying $\frac{d \phi_{t}}{d t}=X_{t} \circ \phi_{t}$ and starting at $\phi_{0}=$ identity. Thus, one has $\phi_{1}^{*} \omega_{1}=\phi_{0}^{*} \omega_{0}=\omega_{0}$, so $\phi_{1}$ provides a chart describing the evolution from $\omega_{0}$ to $\omega_{1}=\omega_{0}+d A$. In terms of local coordinates, there always exists a coordinate transformation $\phi_{1}$ whose pullback maps $\omega_{1}=\omega_{0}+d A$ to $\omega_{0}$, i.e., 
$\phi_{1}: y \mapsto x=x(y)$ so that

$$
\frac{\partial x^{a}}{\partial y^{\mu}} \frac{\partial x^{b}}{\partial y^{\nu}} \omega_{1 a b}(x)=\omega_{0 \mu \nu}(y) .
$$

This can directly be applied to the open string case, Eq. (23), by considering a local Darboux chart $\left(U ; y^{1}, \cdots, y^{2 n}\right)$ centered at $p \in U$ and valid on an open neighborhood $U \subset M$ such that $\left.\omega_{0}\right|_{U}=\frac{1}{2} B_{\mu \nu} d y^{\mu} \wedge d y^{\nu}$, where $B_{\mu \nu}$ is a constant symplectic matrix of rank $2 n$. Now, consider a flow $\phi_{t}: U \times[0,1] \rightarrow M$ generated by the vector field $X_{t}$ satisfying Eq. (28). Under the action of $\phi_{\epsilon}$ with an infinitesimal $\epsilon$, one finds that the point $p \in U$ whose coordinates are $y^{\mu}$ is mapped to $\phi_{\epsilon}(y) \equiv x^{\mu}(y)=y^{\mu}+\epsilon X^{\mu}(y)$. Using the inverse map $\phi_{\epsilon}^{-1}: x^{\mu} \mapsto y^{\mu}(x)=x^{\mu}-\epsilon X^{\mu}(x)$, the symplectic structure $\left.\omega_{0}\right|_{U}=\frac{1}{2} B_{\mu \nu}(y) d y^{\mu} \wedge d y^{\nu}$ can be expressed as

$$
\begin{aligned}
\left(\phi_{\epsilon}^{-1}\right)^{*}\left(\left.\omega_{0}\right|_{y}\right) & =\frac{1}{2} B_{\mu \nu}(x-\epsilon X) d\left(x^{\mu}-\epsilon X^{\mu}\right) \wedge d\left(x^{\nu}-\epsilon X^{\nu}\right) \\
& \approx \frac{1}{2}\left[B_{\mu \nu}-\epsilon X^{a}\left(\partial_{a} B_{\mu \nu}+\partial_{\nu} B_{a \mu}+\partial_{\mu} B_{\nu a}\right)+\epsilon\left(\partial_{\mu}\left(B_{\nu a} X^{a}\right)-\partial_{\nu}\left(B_{\mu a} X^{a}\right)\right)\right] d x^{\mu} \wedge d x^{\nu} \\
& \equiv B+\epsilon F
\end{aligned}
$$

where $A_{\mu}(x)=B_{\mu a}(x) X^{a}(x)$ or $\iota_{X} B+A=0$ and $d B=0$ was used so that the second term vanished. Equation (30) can be rewritten as $\phi_{\epsilon}^{*}(B+\epsilon F)=B$, which means that the electromagnetic force $F=d A$ can always be eliminated by a local coordinate transformation generated by the vector field $X$ satisfying Eq. (28).

Now, let us go back to the previous question. We considered a symmetry transformation which is a combination of the $\Lambda$-transformation, Eq. (25), followed by a diffeomorphism $\phi: N \rightarrow M$. It transforms the DBI metric $g+\kappa B$ on $M$ according to $g+\kappa B \rightarrow \phi^{*}(g+\kappa \Omega)$. The crux is that there exists a diffeomorphism $\phi: N \rightarrow M$ such that $\phi^{*}(\Omega)=B$, which is precisely the Darboux transformation in Eq. (29) in symplectic geometry. Then, we arrive at a remarkable fact [7] that two different DBI metrics, $g+\kappa \Omega$ and $G+\kappa B$, are diffeomorphic to each other, i.e.,

$$
\phi^{*}(g+\kappa \Omega)=G+\kappa B
$$

where $G=\phi^{*}(g)$. Because the open string theory, Eq. (23), respects the diffeomorphism symmetry, Eq. (24), the D-manifolds described by $(M, g, \Omega)$ and $(N, G, B)$ must be physically equivalent. Note that this property holds for any pair $(g, B)$ of a Riemannian metric $g$ and a symplectic structure $B$.

The above argument reveals superb physics in gauge theory. There "always" exists a coordinate transformation to locally eliminate the electromagnetic force $F=d A$ as long as a manifold $M$ 
supports a symplectic structure $B$; i.e., $(M, B)$ defines a symplectic manifold. That is, a symplectic structure on a spacetime manifold $M$ admits a novel form of the equivalence principle, known as the Darboux theorem, for the geometrization of the electromagnetic force. Because it is always possible to find a coordinate transformation $\phi \in \operatorname{Diff}(M)$ such that $\phi^{*}(B+F)=B$, the relationship $\phi^{*}(g+\kappa(B+F))=G+\kappa B$ clearly shows that the electromagnetic fields in the DBI metric $g+\kappa(B+F)$ now appear as a new metric $G=\phi^{*}(g)$ after the Darboux transformation in Eq. (31). One may also consider the inverse relationship $\phi_{*}(G+\kappa B)=g+\kappa(B+F)$, which implies that a nontrivial metric $G$ in the background $B$ can be interpreted as an inhomogeneous condensation of gauge fields on a 'D-manifold' with metric $g$. We might point out that the relationship in the case of $\kappa=2 \pi \alpha^{\prime}=0$ is the familiar diffeomorphism in a Riemannian geometry, so it says nothing marvelous. See footnote [13].

We observed that the Darboux transformation between symplectic structures immediately leads to the diffeomorphism between two different DBI metrics. In terms of local coordinates $\phi: y \mapsto$ $x=x(y)$, the diffeomorphism in Eq. (31) explicitly reads as

$$
(g+\kappa \Omega)_{a b}(x)=\frac{\partial y^{\mu}}{\partial x^{a}}\left(G_{\mu \nu}(y)+\kappa B_{\mu \nu}(y)\right) \frac{\partial y^{\nu}}{\partial x^{b}},
$$

where $\Omega=B+F$ and

$$
G_{\mu \nu}(y)=\frac{\partial x^{a}}{\partial y^{\mu}} \frac{\partial x^{b}}{\partial y^{\nu}} g_{a b}(x)
$$

Equation (32) conclusively shows how gauge fields on a symplectic manifold manifest themselves as a spacetime geometry. To expose the intrinsic connection between gauge fields and spacetime geometry, let us represent the coordinate transformation in Eq. (32) as

$$
x^{a}(y)=y^{a}+\theta^{a b} \widehat{A}_{b}(y)
$$

with $\theta^{a b}=\left(B^{-1}\right)^{a b}$. Note that the coordinate transformation in Eq. (34) for the case $F(x)=0$ is trivial, i.e., $\widehat{A}_{a}(y)=0$ and $G_{a b}=g_{a b}$ as it should be, while it is nontrivial for $F(x) \neq 0$. The nontrivial coordinate transformation can be encoded into smooth functions $\widehat{A}_{a}(y)$, which will be identified with noncommutative gauge fields. Clearly, Eq. (33) embodies how the metric on $M$ is deformed by the presence of noncommutative gauge fields.

We showed how the diffeomorphism symmetry in Eq. (31) between two different DBI metrics arises from $U(1)$ gauge theory on a symplectic manifold. Surprisingly (at least to us), the diffeomorphism symmetry in Eq. (31) is realized as a novel form of the equivalence principle 
for the electromagnetic force [5]. Therefore, one may expect electromagnetism on a symplectic manifold to be a theory of gravity; in other words, gravity can emerge from electromagnetism on a symplectic or Poisson manifold.

A low-energy effective field theory deduced from the open string action in Eq. (23) describes an open string dynamics on a $(p+1)$-dimensional D-brane worldvolume [11]. The dynamics of D-branes is described by open string field theory whose low-energy effective action is obtained by integrating out all the massive modes and keeping only massless fields that are slowly varying at the string scale $\kappa=2 \pi l_{s}^{2}$. For a $D p$-brane in closed string background fields, the action describing the resulting low-energy dynamics is given by

$$
S=\frac{2 \pi}{g_{s}(2 \pi \kappa)^{\frac{p+1}{2}}} \int d^{p+1} x \sqrt{\operatorname{det}(g+\kappa(B+F))}+\mathcal{O}(\sqrt{\kappa} \partial F, \cdots),
$$

where $F=d A$ is the field strength of $U(1)$ gauge fields. The DBI action in Eq. (35) respects the two local symmetries, Eqs. (24) and (25), as expected.

Note that ordinary $U(1)$ gauge symmetry is a special case of Eq. (25) where the gauge parameter $\Lambda$ is exact, namely, $\Lambda=d \lambda$, so that $B \rightarrow B$ and $A \rightarrow A+d \lambda$. One can see from Eq. (28) that the $U(1)$ gauge transformation is generated by a Hamiltonian vector field $X_{\lambda}$ satisfying $\iota_{X_{\lambda}} B+d \lambda=0$. Therefore, the gauge symmetry acting on $U(1)$ gauge fields as $A \rightarrow A+d \lambda$ is a diffeomorphism symmetry generated by the vector field $X_{\lambda}$ satisfying $\mathcal{L}_{X_{\lambda}} B=0$, which is known to be a symplectomorphism. We see here that the $U(1)$ gauge symmetry on the symplectic manifold $(M, B)$ turns into a "spacetime" symmetry rather than an "internal" symmetry. This fact already implies an intimate connection between gauge fields on a symplectic manifold and spacetime geometry.

It was shown in Eq. (32) that the strong isotopy in Eq. (29) between symplectic structures brings in the diffeomorphic equivalence, Eq. (32), between two different DBI metrics, which, in turn, leads to a remarkable identity [56] between DBI actions:

$$
\int_{M} d^{p+1} x \sqrt{\operatorname{det}(g(x)+\kappa(B+F)(x))}=\int_{N} d^{p+1} y \sqrt{\operatorname{det}(G(y)+\kappa B(y))} .
$$

The property in Eq. (36) appearing in the geometrization of electromagnetism may be summarized in the context of a derived category. More closely, if $M$ is a complex manifold whose complex structure is given by $J$, we see that dynamical fields on the left-hand side of Eq. (36) act only as the deformation of the symplectic structure $\Omega(x)=(B+F)(x)$ in the triple $(M, J, \Omega)$ while those on the right-hand side appear only as the deformation of the complex structure $K=\phi^{*}(J)$ in the 
triple $(N, K, B)$ through the metric in Eq. (33). In this notation, the identity in Eq. (36) can, thus, be written as follows:

$$
(M, J, \Omega) \cong(N, K, B)
$$

The equivalence, Eq. (37), is very reminiscent of the homological mirror symmetry [43], which states the equivalence between the category of A-branes (derived Fukaya category corresponding to the triple $(M, J, \Omega)$ ) and the category of B-branes (derived category of coherent sheaves corresponding to the triple $(N, K, B)$ ).

Because the open string action in Eq. (23) basically describes a $U(1)$-bundle (the Chan-Paton bundle) on a D-brane whose data are given by $(M, g, B), U(1)$ gauge fields, the connections of the $U(1)$ bundle are regarded as dynamical fields while the metric $g$ and the two-form $B$ are considered as background fields. However, Eq. (36) clearly shows that gauge field fluctuations can be interpreted as a dynamical metric on the brane given by Eq. (33). In all, one may wonder whether the right-hand side of Eq. (36) can be rewritten as a theory of gravity. Remarkably, it is the case, as will be shown soon.

Here, we will use the background-independent prescription [16] in which the open string metric $\widehat{g}_{a b}$, the noncommutativity $\theta^{a b}$ and the open string coupling constant $\widehat{g}_{s}$ are determined by

$$
\theta^{a b}=\left(\frac{1}{B}\right)^{a b}, \quad \widehat{g}_{a b}=-\kappa^{2}\left(B \frac{1}{g} B\right)_{a b}, \quad \widehat{g}_{s}=g_{s} \sqrt{\operatorname{det}\left(\kappa B g^{-1}\right)} .
$$

In terms of these parameters, the couplings are related by

$$
\begin{aligned}
& \frac{1}{g_{Y M}^{2}}=\frac{\kappa^{2-n}}{(2 \pi)^{n-1} \widehat{g}_{s}}, \\
& \frac{\sqrt{\operatorname{det} \widehat{g}}}{\widehat{g}_{s}}=\frac{\kappa^{n}}{g_{s}|\operatorname{Pf} \theta|},
\end{aligned}
$$

where $p+1 \equiv 2 n$. For constant $g$ and $B$, one can rewrite the right-hand side of Eq. (36) by using the open string variables and defining new covariant fields $D_{a}(y) \equiv B_{a b} x^{b}(y)$ as

$$
\int d^{p+1} y \sqrt{\operatorname{det}(G(y)+\kappa B)}=\int d^{p+1} y \sqrt{\operatorname{det}\left(\kappa B+\kappa^{2} \widehat{G}(y)\right)},
$$

where

$$
\widehat{G}_{\mu \nu}(y)=\widehat{g}^{a b} \frac{\partial D_{a}}{\partial y^{\mu}} \frac{\partial D_{b}}{\partial y^{\nu}}
$$


One can expand the right-hand side of Eq. (41) around the background $B$ in powers of $\kappa$, arriving at the following result:

$$
\begin{aligned}
& \int d^{p+1} y \sqrt{\operatorname{det}\left(\kappa B+\kappa^{2} \widehat{G}(y)\right)} \\
& \quad=\int d^{p+1} y \sqrt{\operatorname{det}(\kappa B)}\left(1+\frac{\kappa^{2}}{4} \widehat{g}^{a c} \widehat{g}^{b d}\left\{D_{a}, D_{b}\right\}_{\theta}\left\{D_{c}, D_{d}\right\}_{\theta}+\cdots\right)
\end{aligned}
$$

where $\left\{D_{a}, D_{b}\right\}_{\theta}$ is the Poisson bracket defined by Eq. (6). The second part of Eq. (43) can then be written in a form with a constant metric $\widehat{g}^{a b}=-\frac{1}{\kappa^{2}}(\theta g \theta)^{a b}$ as

$$
S_{D}=\frac{1}{4 g_{Y M}^{2}} \int d^{p+1} y \sqrt{\operatorname{det} \widehat{g}} \widehat{g}^{a c} \widehat{g}^{b d}\left\{D_{a}, D_{b}\right\}_{\theta}\left\{D_{c}, D_{d}\right\}_{\theta},
$$

where the gauge coupling constant $g_{Y M}$ was recovered after collecting all factors including the one in Eq. (35). From now on, let us set the metric $\widehat{g}^{a b}=\delta^{a b}$ for simplicity.

Note that

$$
\begin{aligned}
\left\{D_{a}, D_{b}\right\}_{\theta} & =-B_{a b}+\partial_{a} \widehat{A}_{b}-\partial_{b} \widehat{A}_{a}+\left\{\widehat{A}_{a}, \widehat{A}_{b}\right\}_{\theta} \\
& \equiv-B_{a b}+\widehat{F}_{a b}
\end{aligned}
$$

and

$$
\begin{aligned}
\left\{D_{a},\left\{D_{b}, D_{c}\right\}_{\theta}\right\}_{\theta} & =\partial_{a} \widehat{F}_{b c}+\left\{\widehat{A}_{a}, \widehat{F}_{b c}\right\}_{\theta} \\
& \equiv \widehat{D}_{a} \widehat{F}_{b c}
\end{aligned}
$$

Therefore, the Jacobi identity for the Poisson bracket in Eq. (6) can be written as

$$
\begin{aligned}
0 & =\left\{D_{a},\left\{D_{b}, D_{c}\right\}_{\theta}\right\}_{\theta}+\left\{D_{b},\left\{D_{c}, D_{a}\right\}_{\theta}\right\}_{\theta}+\left\{D_{c},\left\{D_{a}, D_{b}\right\}_{\theta}\right\}_{\theta} \\
& =\widehat{D}_{a} \widehat{F}_{b c}+\widehat{D}_{b} \widehat{F}_{c a}+\widehat{D}_{c} \widehat{F}_{a b}
\end{aligned}
$$

Similarly, the equations of motion derived from the action in Eq. (44) read as

$$
\left\{D^{a},\left\{D_{a}, D_{b}\right\}_{\theta}\right\}_{\theta}=\widehat{D}^{a} \widehat{F}_{a b}=0
$$

Note that electromagnetism on a symplectic manifold is a nonlinear interacting theory as the selfinteraction in Eq. (45) clearly shows.

Going from the left-hand side of Eq. (36) to the right-hand side, we have eliminated the $U(1)$ gauge field in terms of the local coordinate transformation in Eq. (34). Nevertheless, if one looks at the action in Eq. (44), which was obtained by expanding the DBI action free from gauge fields, 
gauge fields seem to appear again on first look. One may thus suspect that the action in Eq. (44) does not satisfy the equivalence principle we have justified before. However, one has to notice that the gauge fields in Eq. (34) should be regarded as dynamical coordinates describing a fluctuating metric as in Eq. (33). Rather an interesting point is that the fluctuation of the emergent metric, Eq. (33), can be written in the form of gauge theory on a symplectic spacetime. This highlights a key feature in realizing the gauge/gravity duality in noncommutative spacetime.

One can identify the defining fields $D_{a}(y) \in C^{\infty}(M), a=1, \cdots, p+1=2 n$, in the action in Eq. (44) with the set in Eq. (11) and, according to the map in Eq. (12), the fields $D_{a}(y)$ can be mapped to vector fields $V_{a} \in \Gamma(T M)$. One can immediately see by identifying $f=D_{a}$ and $g=D_{b}$ and by using the relation in Eq. (45) that the Lie algebra homomorphism in Eq. (10) leads to the following identity:

$$
X_{\widehat{F}_{a b}}=\left[V_{a}, V_{b}\right],
$$

where $V_{a} \equiv X_{D_{a}}$ and $V_{b} \equiv X_{D_{b}}$. Similarly, using Eq. (46), one can further deduce that

$$
X_{\widehat{D}_{a} \widehat{F}_{b c}}=\left[V_{a},\left[V_{b}, V_{c}\right]\right] .
$$

Then, the map in Eq. (50) translates the Jacobi identity in Eq. (47) and the equations of motion in Eq. (48) into some relations between the vector fields $V_{a}$ defined by Eq. (12). That is, we have the following correspondence [7]:

$$
\begin{aligned}
& \widehat{D}_{[a} \widehat{F}_{b c]}=0 \quad \Leftrightarrow \quad\left[V_{[a},\left[V_{b}, V_{c]}\right]\right]=0, \\
& \widehat{D}^{a} \widehat{F}_{a b}=0 \quad \Leftrightarrow \quad\left[V^{a},\left[V_{a}, V_{b}\right]\right]=0,
\end{aligned}
$$

where $[a, b, c]$ denotes a fully antisymmetrization of indices $(a, b, c)$ and the bracket between the vector fields on the right-hand side is defined by the Lie bracket.

As we remarked before, the vector fields $V_{a} \in \Gamma(T M)$ can be related to the vielbeins $E_{a} \in$ $\Gamma(T M)$ in Eq. (2) by $V_{a}=\lambda E_{a}$, with $\lambda \in C^{\infty}(M)$ to be determined, so the double Lie brackets in Eqs. (51) and (52) will be related to Riemann curvature tensors because they are involved with the second-order derivatives of the metric in Eq. (11) or the vielbein in Eq. (2). It will be rather straightforward to derive Einstein gravity from the set of equations, Eqs. (51) and (52), which is the subject of the following two subsections. 


\section{B. Warm-up with a Beautiful Example}

First, let us briefly summarize some aspects in differential geometry [3] that are useful in understanding some concepts of emergent gravity. Let $E^{a}=E_{\mu}^{a} d y^{\mu}$ be the basis of 1-forms dual to a given frame $E_{a}=E_{a}^{\mu} \partial_{\mu}$. If we define the local matrix of connection 1-forms by

$$
\omega_{b}^{a}=\omega_{\mu b}^{a} d y^{\mu}=\omega_{c b}^{a} E^{c},
$$

the first Cartan's structure equation

$$
T^{a}=d E^{a}+\omega^{a}{ }_{b} \wedge E^{b}
$$

describes the torsion as a 2-form in terms of the vielbein basis, and the second structure equation

$$
R_{b}^{a}=d \omega_{b}^{a}+\omega^{a}{ }_{c} \wedge \omega_{b}^{c}
$$

allows to compute the matrix-valued curvature 2 -form by using the connection.

The metric compatibility leads to the symmetry $\omega_{a b}=-\omega_{b a}$, which, together with the torsionfree condition $T^{a}=0$, uniquely determines the connection 1-form, Eq. (53), which is nothing other than the Levi-Civita connection in the vielbein formalism

$$
\begin{aligned}
\omega_{a b c} & =\frac{1}{2}\left(f_{a b c}-f_{b c a}+f_{c a b}\right) \\
& =-\omega_{a c b},
\end{aligned}
$$

where

$$
f_{a b c}=E_{a}^{\mu} E_{b}^{\nu}\left(\partial_{\mu} E_{\nu c}-\partial_{\nu} E_{\mu c}\right)
$$

are the structure functions of the vectors $E_{a} \in \Gamma(T M)$ defined by Eq. (3).

Now, let us specialize to a Riemannian four-manifold $M$. Because the spin connection $\omega_{\mu a b}$ and the curvature tensor $R_{\mu \nu a b}$ are antisymmetric on the $a b$ index pair, one can decompose them into a self-dual part and an anti-self-dual part as follows [7]:

$$
\begin{aligned}
& \omega_{\mu a b}=A_{\mu}^{(+) i} \eta_{a b}^{i}+A_{\mu}^{(-) i} \bar{\eta}_{a b}^{i}, \\
& R_{\mu \nu a b}=F_{\mu \nu}^{(+) i} \eta_{a b}^{i}+F_{\mu \nu}^{(-) i} \bar{\eta}_{a b}^{i},
\end{aligned}
$$

where the $4 \times 4$ matrices $\eta_{a b}^{i} \equiv \eta_{a b}^{(+) i}$ and $\bar{\eta}_{a b}^{i} \equiv \eta_{a b}^{(-) i}$ for $i=1,2,3$ are 't Hooft symbols defined by

$$
\begin{aligned}
& \bar{\eta}_{j k}^{i}=\eta_{j k}^{i}=\varepsilon_{i j k}, \quad j, k \in\{1,2,3\}, \\
& \bar{\eta}_{4 j}^{i}=\eta_{j 4}^{i}=\delta_{i j} .
\end{aligned}
$$


Note that the 't Hooft matrices intertwine the group structure of index $i$ with the spacetime structure of indices $a, b$. See appendix A in Ref. [7] for some useful identities for the 't Hooft tensors.

Any $S O(4)=S U(2)_{L} \times S U(2)_{R} / \mathbf{Z}_{2}$ rotations can be decomposed into self-dual and anti-selfdual rotations. Let us introduce two families of $4 \times 4$ matrices defined by

$$
\left(T_{+}^{i}\right)_{a b} \equiv \eta_{a b}^{i}, \quad\left(T_{-}^{i}\right)_{a b} \equiv \bar{\eta}_{a b}^{i}
$$

Then, one can show that $T_{ \pm}^{i}$ satisfy $S U(2)$ Lie algebras, i.e.,

$$
\left[T_{ \pm}^{i}, T_{ \pm}^{j}\right]=-2 \varepsilon^{i j k} T_{ \pm}^{k}, \quad\left[T_{ \pm}^{i}, T_{\mp}^{j}\right]=0
$$

Indeed the 't Hooft tensors in Eq. (61) are two independent spin $s=\frac{3}{2}$ representations of the $S U(2)$ Lie algebra. A deep geometric meaning of the 't Hooft tensors is to specify the triple $(I, J, K)$ of complex structures of $\mathbb{R}^{4} \cong \mathbb{C}^{2}$ for a given orientation as the simplest hyper-Kähler manifold. The triple complex structures $(I, J, K)$ form a quaternion, that can be identified with the $S U(2)$ generators $T_{ \pm}^{i}$ in Eq. (61).

Using the representation $\omega_{\mu a b}^{( \pm)}=A_{\mu}^{( \pm) i}\left(T_{ \pm}^{i}\right)_{a b}=\left(A_{\mu}^{( \pm)}\right)_{a b}$ in Eq. (58), we can write the (anti)self-dual curvature tensors in Eq. (59) in the form

$$
F_{\mu \nu}^{( \pm)}=\partial_{\mu} A_{\nu}^{( \pm)}-\partial_{\nu} A_{\mu}^{( \pm)}+\left[A_{\mu}^{( \pm)}, A_{\nu}^{( \pm)}\right]
$$

Therefore, we see that $A_{\mu}^{( \pm)}$can be identified with $S U(2)_{L, R}$ gauge fields and $F_{\mu \nu}^{( \pm)}$with their field strengths. Indeed, one can also show that the local $S O(4)$ rotations

$$
\omega_{\mu} \rightarrow \Lambda \omega_{\mu} \Lambda^{-1}+\Lambda \partial_{\mu} \Lambda^{-1}
$$

with $\Lambda_{b}^{a}(y) \in S O(4)$ can be represented as the gauge transformations of the $S U(2)$ gauge field $A_{\mu}^{( \pm)}$

$$
A_{\mu}^{( \pm)} \rightarrow \Lambda_{ \pm} A_{\mu}^{( \pm)} \Lambda_{ \pm}^{-1}+\Lambda_{ \pm} \partial_{\mu} \Lambda_{ \pm}^{-1}
$$

where $\Lambda_{ \pm} \in S U(2)_{L, R}[$ [57].

With the form language where $d=d y^{\mu} \partial_{\mu}=E^{a} E_{a}$ and $A=A_{\mu} d y^{\mu}=A_{a} E^{a}$, the field strength, Eq. [63), of $S U(2)$ gauge fields in a coordinate basis is given by

$$
\begin{aligned}
F^{( \pm)} & =d A^{( \pm)}+A^{( \pm)} \wedge A^{( \pm)} \\
& =\frac{1}{2} F_{\mu \nu}^{( \pm)} d y^{\mu} \wedge d y^{\nu} \\
& =\frac{1}{2}\left(\partial_{\mu} A_{\nu}^{( \pm)}-\partial_{\nu} A_{\mu}^{( \pm)}+\left[A_{\mu}^{( \pm)}, A_{\nu}^{( \pm)}\right]\right) d y^{\mu} \wedge d y^{\nu}
\end{aligned}
$$


or in a non-coordinate basis by

$$
\begin{aligned}
F^{( \pm)} & =\frac{1}{2} F_{a b}^{( \pm)} E^{a} \wedge E^{b} \\
& =\frac{1}{2}\left(E_{a} A_{b}^{( \pm)}-E_{b} A_{a}^{( \pm)}+\left[A_{a}^{( \pm)}, A_{b}^{( \pm)}\right]+f_{a b}^{c} A_{c}^{( \pm)}\right) E^{a} \wedge E^{b}
\end{aligned}
$$

where we used in Eq. 67) the structure equation

$$
d E^{a}=\frac{1}{2} f_{b c}^{a} E^{b} \wedge E^{c}
$$

As we remarked, the 't Hooft tensors $\left\{\eta_{a b}^{( \pm) i}\right\}$ specify the triple of complex structures of the simplest hyper-Kähler manifold $\mathbb{R}^{4}$ satisfying the quaternion algebra. Because any Riemannian metric can be written as $g_{\mu \nu}(y)=E_{\mu}^{a}(y) E_{\nu}^{b}(y) \delta_{a b}$, one can introduce the corresponding triple of local complex structures on the Riemannian manifold $M$

$$
J^{( \pm) i}=\frac{1}{2} \eta_{a b}^{( \pm) i} E^{a} \wedge E^{b}
$$

which are inherited from $\mathbb{R}^{4}$, or in terms of local coordinates

$$
J^{( \pm) i}=\frac{1}{2} \eta_{a b}^{( \pm) i} E_{\mu}^{a} E_{\nu}^{b} d y^{\mu} \wedge d y^{\nu} \equiv \frac{1}{2} J_{\mu \nu}^{( \pm) i} d y^{\mu} \wedge d y^{\nu}
$$

One can easily check that the local complex structures $J^{( \pm) i}$ still satisfy the quaternion algebra

$$
\begin{aligned}
{\left[J^{( \pm) i}, J^{( \pm) j}\right]_{\mu \nu} } & \equiv J_{\mu \lambda}^{( \pm) i} J_{\nu}^{( \pm) j \lambda}-J_{\mu \lambda}^{( \pm) j} J_{\nu}^{( \pm) i \lambda} \\
& =-2 \varepsilon^{i j k} J_{\mu \nu}^{( \pm) k} \\
{\left[J^{( \pm) i}, J^{(\mp) j}\right]_{\mu \nu} } & =0 .
\end{aligned}
$$

Now, it is easy to see that the torsion-free condition $T^{a}=0$ is equivalent to the one in which the complex structures $J^{( \pm) i}$ are covariantly constant, i.e.,

$$
\begin{aligned}
d J^{( \pm) i} & =\frac{1}{2} \eta_{a b}^{( \pm) i} d E^{a} \wedge E^{b}-\frac{1}{2} \eta_{a b}^{( \pm) i} E^{a} \wedge d E^{b} \\
& =-\left[\eta^{( \pm) i} \eta^{(\mp) j}\right]_{a b} A^{(\mp) j} \wedge E^{a} \wedge E^{b}+2 \varepsilon^{i j k} A^{( \pm) j} \wedge J^{( \pm) k} \\
& =2 \varepsilon^{i j k} A^{( \pm) j} \wedge J^{( \pm) k},
\end{aligned}
$$

where we used the fact that $\left[\eta^{( \pm) i} \eta^{(\mp) j}\right]_{a b}$ is symmetric, i.e., $\left[\eta^{( \pm) i} \eta^{(\mp) j}\right]_{a b}=\left[\eta^{( \pm) i} \eta^{(\mp) j}\right]_{b a}$. Therefore, we get the relation

$$
d_{A} J^{( \pm) i} \equiv d J^{( \pm) i}-2 \varepsilon^{i j k} A^{( \pm) j} \wedge J^{( \pm) k}=0 .
$$


All these properties can be beautifully summarized using the Palatini formalism of Einstein gravity, in which the spin connection and the vielbein are regarded as independent variables. The Einstein-Hilbert action in the Palatini formalism is given by

$$
S_{P}=\frac{1}{4} \int_{M} \varepsilon_{a b}^{c d} E^{a} \wedge E^{b} \wedge R_{c d}
$$

By varying the action in Eq. (74) with respect to the vierbein and the spin connection, one can get the torsion-free condition $T^{a}=d E^{a}+\omega^{a}{ }_{b} \wedge E^{b}=0$, as well as the Einstein equation $R_{a b}-\frac{1}{2} \delta_{a b} R=$ 0, thus recovering the Einstein gravity.

If the decomposition in Eq. (59) is used, the Palatini action in Eq. (74) can be recast into the beautiful form

$$
\begin{aligned}
S_{P} & =\frac{1}{4} \int_{M} \varepsilon_{a b}^{c d} E^{a} \wedge E^{b} \wedge R_{c d} \\
& =\frac{1}{4} \int_{M} \varepsilon_{a b}^{c d} E^{a} \wedge E^{b} \wedge\left(F^{(+) i} \eta_{c d}^{i}+F^{(-) i} \bar{\eta}_{c d}^{i}\right) \\
& =\frac{1}{2} \int_{M} E^{a} \wedge E^{b} \wedge\left(F^{(+) i} \eta_{a b}^{i}-F^{(-) i} \bar{\eta}_{a b}^{i}\right) \\
& =\int_{M}\left(J^{(+) i} \wedge F^{(+) i}-J^{(-) i} \wedge F^{(-) i}\right) .
\end{aligned}
$$

The action in Eq. (75) immediately shows that the condition in Eq. (73) is, indeed, the equations of motion for $S U(2)$ gauge fields $A^{( \pm) i}$. Interestingly, the Palatini action in Eq. (75) is invariant under a local deformation given by

$$
A^{( \pm) i} \rightarrow A^{( \pm) i}, \quad J^{( \pm) i} \rightarrow J^{( \pm) i}+d_{A} \Lambda^{( \pm) i}
$$

with an arbitrary one-form $\Lambda^{( \pm)} \in S U(2)$. The deformation symmetry in Eq. (76) should be true due to the Bianchi identity $d_{A} F^{( \pm)}=0$.

The gravitational instantons [58] are defined by the self-dual solution to the Einstein equation

$$
R_{e f a b}= \pm \frac{1}{2} \varepsilon_{a b}^{c d} R_{e f c d}
$$

Note that a metric satisfying the self-duality equation, Eq. (77), is necessarily Ricci-flat because $R_{a b}=R_{a c b}{ }^{c}= \pm \frac{1}{6} \varepsilon_{b}{ }^{c d e} R_{a[c d e]}=0$ and thus automatically satisfies the vacuum Einstein equations. If the decomposition in Eq. (59) is used, Eq. (77) can be written as

$$
\begin{aligned}
F_{e f}^{(+) i} \eta_{a b}^{i}+F_{e f}^{(-) i} \bar{\eta}_{a b}^{i} & = \pm \frac{1}{2} \varepsilon_{a b}^{c d}\left(F_{e f}^{(+) i} \eta_{c d}^{i}+F_{e f}^{(-) i} \bar{\eta}_{c d}^{i}\right) \\
& = \pm\left(F_{e f}^{(+) i} \eta_{a b}^{i}-F_{e f}^{(-) i} \bar{\eta}_{a b}^{i}\right) .
\end{aligned}
$$


Therefore, we should have $F_{a b}^{(-) i}=0$ for the self-dual case with a plus sign in Eq. (77) and $F_{a b}^{(+) i}=0$ for the anti-self-dual case with a minus sign, thus imposing the self-duality equation, Eq. (77), is equivalent to the half-flat equation $F^{( \pm) i}=0$. Because the Riemann curvature tensors satisfy the symmetry property

$$
R_{a b c d}=R_{c d a b}
$$

one can rewrite the self-duality equation, Eq. (77), as follows:

$$
R_{a b e f}= \pm \frac{1}{2} \varepsilon_{a b}^{c d} R_{c d e f}
$$

Then, using the decomposition in Eq. (59) again, one can similarly show [59] that the gravitational instanton in Eq. (80) can be understood as an $S U(2)$ Yang-Mills instanton, i.e.,

$$
F_{a b}^{( \pm)}= \pm \frac{1}{2} \varepsilon_{a b}^{c d} F_{c d}^{( \pm)}
$$

where $F_{a b}^{( \pm)}=F_{a b}^{( \pm) i} T_{ \pm}^{i}=E_{a}^{\mu} E_{b}^{\nu} F_{\mu \nu}^{( \pm)}$are defined by Eq. (67).

A solution to the half-flat equation $F^{( \pm)}=0$ is given by $A^{( \pm)}=\Lambda_{ \pm} d \Lambda_{ \pm}^{-1}$; then, Eq. (65) shows that it is always possible to choose a self-dual gauge $A^{( \pm) i}=0$. In this gauge, Eq. (73) reduces to the property $d J^{( \pm) i}=0$; that is, the triple complex structures in one of the $( \pm)$-sectors are all closed. In other words, there is the triple $\left\{J^{( \pm) i}\right\}$ of globally well-defined complex structures. This means that the metric $d s^{2}=E^{a} \otimes E^{a}$ describes a hyper-Kähler manifold. In the end, the gravitational instantons defined by Eq. (77) can be characterized by the following property:

$$
F^{( \pm) i}=0 \quad \Leftrightarrow \quad d J^{( \pm) i}=0, \quad \forall i=1,2,3 .
$$

In order to solve the equations in Eq. (82), let us introduce linearly-independent four-vector fields $V_{a}$ and a volume form $\nu$ on $M$. Then, one can easily check [60] that the (anti-)self-dual ansatz [61]

$$
J^{( \pm) i}=\frac{1}{2} \eta_{a b}^{( \pm) i} \iota_{a} \iota_{b} \nu
$$

where $\iota_{a}$ denotes the inner derivation with respect to $V_{a}$, immediately solves the equations $d J^{( \pm) i}=$ 0 if and only if the vector fields satisfy the following equations [62]

$$
\begin{aligned}
& \frac{1}{2} \eta_{a b}^{( \pm) i}\left[V_{a}, V_{b}\right]=0, \\
& \mathcal{L}_{V_{a}} \nu=0, \quad \forall a=1, \cdots, 4 .
\end{aligned}
$$


This can simply be seen by applying the formula [63]

$$
d\left(\iota_{X} \iota_{Y} \alpha\right)=\iota_{[X, Y]} \alpha+\iota_{Y} \mathcal{L}_{X} \alpha-\iota_{X} \mathcal{L}_{Y} \alpha+\iota_{X} \iota_{Y} d \alpha
$$

for vector fields $X, Y$ and a $p$-form $\alpha$.

Now go back to the action in Eq. (44) and consider the self-duality equation of $U(1)$ gauge fields defined by

$$
\widehat{F}_{a b}= \pm \frac{1}{2} \varepsilon_{a b} c d \widehat{F}_{c d}
$$

Note that the self-duality equation, Eq. (87), is nonlinear due to the Poisson commutator term in Eq. (45), so there exist nontrivial solutions [38]. After quantization, Eq. (7), they become noncommutative $U(1)$ instantons [64]. One can translate the self-duality equation, Eq. [87], to the self-duality equation between vector fields according to the map in Eq. (49):

$$
\widehat{F}_{a b}= \pm \frac{1}{2} \varepsilon_{a b} c d \widehat{F}_{c d} \quad \Leftrightarrow \quad\left[V_{a}, V_{b}\right]= \pm \frac{1}{2} \varepsilon_{a b} c d\left[V_{c}, V_{d}\right]
$$

Recall that the vector fields $V_{a}$ are all divergence-free, i.e., $\partial_{\mu} V_{a}^{\mu}=0$; in other words, $\mathcal{L}_{V_{a}} \nu=0$. Therefore, we see that the self-duality equation, Eq. (87), for gauge fields is certainly equivalent to Eqs. (84) and (85). In conclusion, we finally proved [4-7] the equivalence between $U(1)$ instantons defined by Eq. (87) and gravitational instantons defined by Eq. (77).

\section{Einstein Gravity from Electromagnetism on a Symplectic Manifold}

As a warm-up, we have illustrated with self-dual gauge fields how the Darboux theorem in symplectic geometry implements a deep principle to realize a Riemannian manifold as an emergent geometry from gauge fields on a symplectic manifold through the correspondence in Eq. (12) whose metric is given by

$$
d s^{2}=\delta_{a b} E^{a} \otimes E^{b}=\lambda^{2} \delta_{a b} V_{\mu}^{a} V_{\nu}^{b} d y^{\mu} \otimes d y^{\nu},
$$

where $E^{a}=\lambda V^{a} \in \Gamma\left(T^{*} M\right)$ are dual one-forms. Now, we will generalize the emergent gravity to arbitrary gauge fields on a $2 n$-dimensional symplectic manifold $(M, B)$ and derive Einstein equations from Eqs. (51) and (52).

First let us determine what $\lambda \in C^{\infty}(M)$ in Eq. (89) is. Introduce the structure equation of the vector fields $V_{a}=\lambda E_{a} \in \Gamma(T M)$

$$
\left[V_{a}, V_{b}\right]=-\mathbf{f}_{a b}^{c} V_{c} .
$$


By comparing with Eq. (3), one can get the relation between the two structure functions

$$
\mathbf{f}_{a b}^{c}=\lambda f_{a b}^{c}-V_{a} \log \lambda \delta_{b}^{c}+V_{b} \log \lambda \delta_{a}^{c} .
$$

With the definition in Eq. (90), the self-duality equation, Eq. (88), may be written in a compact form

$$
\eta_{a b}^{( \pm) i} \mathbf{f}_{a b}^{c}=0
$$

Suppose that the $2 n$-dimensional volume form whose four-dimensional example was introduced in Eq. (83) is given by

$$
\nu=\lambda^{2} V^{1} \wedge \cdots \wedge V^{2 n}
$$

in other words,

$$
\lambda^{2}=\nu\left(V_{1}, \cdots, V_{2 n}\right) .
$$

The volume form in Eq. (93) can be related to the Riemannian one $\nu_{g}=E^{1} \wedge \cdots \wedge E^{2 n}$ as

$$
\nu=\lambda^{2-2 n} \nu_{g}
$$

Acting $\mathcal{L}_{V_{a}}$ on both sides of Eq. (94), we get

$$
\begin{aligned}
\mathcal{L}_{V_{a}}\left(\nu\left(V_{1}, \cdots, V_{2 n}\right)\right) & =\left(\mathcal{L}_{V_{a}} \nu\right)\left(V_{1}, \cdots, V_{2 n}\right)+\sum_{b=1}^{2 n} \nu\left(V_{1}, \cdots, \mathcal{L}_{V_{a}} V_{b}, \cdots, V_{2 n}\right) \\
& =\left(\mathcal{L}_{V_{a}} \nu\right)\left(V_{1}, \cdots, V_{2 n}\right)+\sum_{b=1}^{2 n} \nu\left(V_{1}, \cdots,\left[V_{a}, V_{b}\right], \cdots, V_{2 n}\right) \\
& =\left(\nabla \cdot V_{a}+2(1-n) V_{a} \log \lambda+\mathbf{f}_{b a}{ }^{b}\right) \nu\left(V_{1}, \cdots, V_{2 n}\right) \\
& =\left(2 V_{a} \log \lambda\right) \nu\left(V_{1}, \cdots, V_{2 n}\right) .
\end{aligned}
$$

Because $\mathcal{L}_{V_{a}} \nu=\left(\nabla \cdot V_{a}+2(1-n) V_{a} \log \lambda\right) \nu=0$, Eq. (96) leads to the relation

$$
\rho_{a} \equiv \mathbf{f}_{b a}{ }^{b}=2 V_{a} \log \lambda
$$

then, from Eq. (91),

$$
f_{b a}^{b}=(3-2 n) E_{a} \log \lambda
$$


Conversely, if $\mathbf{f}_{b a}{ }^{b}=2 V_{a} \log \lambda$, the vector fields $V_{a}$ preserve the volume form $\nu$, i.e., $\mathcal{L}_{V_{a}} \nu=$ $\left(\nabla \cdot V_{a}+2(1-n) V_{a} \log \lambda\right) \nu=0 \forall a=1, \cdots, 2 n$. Equation (98) implies that the vector fields $E_{a}$ preserve the volume form $\widetilde{\nu}=\lambda^{3-2 n} \nu_{g}$, which can be proven as follows:

$$
\mathcal{L}_{E_{a}}\left(\lambda^{3-2 n} \nu_{g}\right)=d\left(\iota_{E_{a}}\left(\lambda^{3-2 n} \nu_{g}\right)\right)=d\left(\iota_{\lambda E_{a}}\left(\lambda^{2-2 n} \nu_{g}\right)\right)=d\left(\iota_{V_{a}} \nu\right)=\mathcal{L}_{V_{a}} \nu=0 .
$$

In a non-coordinate (anholonomic) basis $\left\{E_{a}\right\}$ satisfying the commutation relation in Eq. (3), the spin connections are defined by

$$
\nabla_{a} E_{c}=\omega_{a}^{b}{ }_{c} E_{b}
$$

where $\nabla_{a} \equiv \nabla_{E_{a}}$ is the covariant derivative in the direction of a vector field $E_{a}$. Acting on the dual basis $\left\{E^{a}\right\}$, they are given by

$$
\nabla_{a} E^{b}=-\omega_{a}^{b}{ }_{c} E^{c}
$$

Because we will impose the torsion-free condition, i.e.,

$$
T(a, b)=\nabla_{[a} E_{b]}-\left[E_{a}, E_{b}\right]=0,
$$

the spin connections are related to the structure functions

$$
f_{a b c}=-\omega_{a c b}+\omega_{b c a} .
$$

The Riemann curvature tensors in the basis $\left\{E_{a}\right\}$ are defined by

$$
R(a, b)=\left[\nabla_{a}, \nabla_{b}\right]-\nabla_{[a, b]}
$$

or in component form, by

$$
\begin{aligned}
& R_{a b d}^{c}=\left\langle E^{c}, R\left(E_{a}, E_{b}\right) E_{d}\right\rangle
\end{aligned}
$$

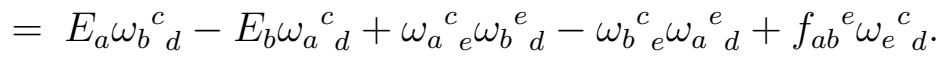

Imposing the condition that the metric in Eq. (89) is covariantly constant, i.e.,

$$
\nabla_{c}\left(\delta_{a b} E^{a} \otimes E^{b}\right)=0
$$

or, equivalently,

$$
\omega_{c a b}=-\omega_{c b a},
$$


we see that the spin connections $\omega_{c a b}$ have the same number of components as $f_{a b c}$. Thus, Eq. (103) has a unique solution, and it is precisely given by Eq. (56). The definition in Eq. (104), together with the metricity condition in Eq. (107), immediately leads to the following symmetry property:

$$
R_{a b c d}=-R_{a b d c}=-R_{b a c d} .
$$

If the relation in Eq. (91) is used, the spin connections in Eq. (56) are now determined by the gauge theory bases

$$
\lambda \omega_{a b c}=\frac{1}{2}\left(\mathbf{f}_{a b c}-\mathbf{f}_{b c a}+\mathbf{f}_{c a b}\right)-V_{b} \log \lambda \delta_{c a}+V_{c} \log \lambda \delta_{a b}
$$

The spacetime geometry described by the metric in Eq. (89) is an emergent gravity arising from gauge fields whose underlying theory is defined by the action in Eq. (44). The fundamental variables in our approach are, of course, gauge fields that are subject to Eqs. (51) and (52). A spacetime metric is now regarded as a collective variable defined by a composite or bilinear of gauge fields. Therefore, we are going to get a viable realization of the idea we speculated about with Eq. (2); is it possible to show that the equations of motion, Eq. (52), for gauge fields together with the Bianchi identity in Eq. (51) can be rewritten as the Einstein equations for the metric in Eq. (89)? For this purpose, we first want to represent the Riemann curvature tensors in Eq. (105), originally expressed with the orthonormal basis $E_{a}$, in terms of the gauge theory basis $V_{a}$. That representation will be useful because we will eventually impose Eqs. (51) and (52) on them.

Indeed, everything is prepared because all calculations can straightforwardly be done using Eqs. (91) and (109). All the details can be found in Ref. [7]. Here, we will briefly sketch essential steps. One can easily derive the following identity:

$$
\begin{aligned}
& R\left(E_{a}, E_{b}\right) E_{c}+R\left(E_{b}, E_{c}\right) E_{a}+R\left(E_{c}, E_{a}\right) E_{b} \\
= & {\left[E_{a},\left[E_{b}, E_{c}\right]\right]+\left[E_{b},\left[E_{c}, E_{a}\right]\right]+\left[E_{c},\left[E_{a}, E_{b}\right]\right] }
\end{aligned}
$$

by using the torsion-free condition in Eq. (102). The Jacobi identity then immediately leads to $R_{[a b c] d}=0$. Because $V_{a}=\lambda E_{a}$, we have the relation

$$
\left[V_{[a},\left[V_{b}, V_{c]}\right]\right]=\lambda^{3}\left[E_{[a},\left[E_{b}, E_{c]}\right]\right]
$$

where all the terms containing the derivatives of $\lambda$ cancel each other. As we promised, the first Bianchi identity $R_{[a b c] d}=0$ follows from the Jacobi identity in Eq. (51). Thus, we pleasingly 
confirm that

$$
\widehat{D}_{[a} \widehat{F}_{b c]}=0 \quad \Leftrightarrow \quad R_{[a b c] d}=0
$$

The Bianchi identity in Eq. (112), together with Eq. (108), leads to the symmetry in Eq. (79). It should be emphasized that the equivalence in Eq. (112) holds for arbitrary gauge fields in any even dimensions.

From the above derivation, we have to notice that, if torsion-free condition in Eq. (102) are not imposed, the equivalence in Eq. (112) must be corrected. This can be seen from the Bianchi identities [3]

$$
\begin{aligned}
& D T^{a} \equiv d T^{a}+\omega^{a}{ }_{b} \wedge T^{b}=R_{b}^{a} \wedge E^{b}, \\
& D R^{a}{ }_{b} \equiv d R^{a}{ }_{b}+\omega^{a}{ }_{c} \wedge R_{b}^{c}-R^{a}{ }_{c} \wedge \omega^{c}{ }_{b}=0,
\end{aligned}
$$

which are integrability conditions derived from Eqs. (54) and (55). In general, the equivalence in Eq. (112) holds only if $D T^{d}=0$, where $R_{c}{ }^{d} \wedge E^{c}=\frac{1}{2} R_{a b c}{ }^{d} E^{a} \wedge E^{b} \wedge E^{c}=\frac{1}{6} R_{[a b c]}{ }^{d} E^{a} \wedge E^{b} \wedge E^{c}=$ 0. Note that Eq. (51) is simply a consequence of the Jocobi identity for the Poisson bracket in Eq. (6), which should be true for a general Poisson structure (not necessarily nondegenerate) as long as the Schouten-Nijenhuis bracket $[\theta, \theta]_{S N} \in \Gamma\left(\Lambda^{3} T M\right)$ vanishes. Thus, Eq. (51) will still be true for a generic Poisson manifold. Also, one may get the relation $R_{[a b c]}^{d}-D_{[a} T_{b c]}^{d}=0$, instead of Eq. (111), for a nonzero torsion. Nevertheless, we conjecture that the torsion will identically vanish even for a general Poisson manifold because the existence of a Poisson structure implies the equivalence principle in the theory of emergent gravity.

The mission for the equations of motion, Eq. (52), is more nontrivial. After some technical manipulation, a remarkably simple form for the Ricci tensor can be obtained in four dimensions [7]:

$$
\begin{aligned}
R_{a b}= & -\frac{1}{\lambda^{2}}\left[\mathbf{f}_{d}^{(+) i} \eta_{a c}^{i} \mathbf{f}_{d}^{(-) j} \bar{\eta}_{b c}^{j}+\mathbf{f}_{d}^{(+) i} \eta_{b c}^{i} \mathbf{f}_{d}^{(-) j} \bar{\eta}_{a c}^{j}\right. \\
& \left.-\left(\mathbf{f}_{c}^{(+) i} \eta_{a c}^{i} \mathbf{f}_{d}^{(-) j} \bar{\eta}_{b d}^{j}+\mathbf{f}_{c}^{(+) i} \eta_{b c}^{i} \mathbf{f}_{d}^{(-) j} \bar{\eta}_{a d}^{j}\right)\right]
\end{aligned}
$$

Here, we also decomposed $\mathbf{f}_{a b c}$ into self-dual and anti-self-dual parts as in Eq. (58):

$$
\mathbf{f}_{a b c}=\mathbf{f}_{c}^{(+) i} \eta_{a b}^{i}+\mathbf{f}_{c}^{(-) i} \bar{\eta}_{a b}^{i},
$$

where

$$
\mathbf{f}_{c}^{( \pm) i} \eta_{a b}^{( \pm) i}=\frac{1}{2}\left(\mathbf{f}_{a b c} \pm \frac{1}{2} \varepsilon_{a b}{ }^{d e} \mathbf{f}_{d e c}\right) .
$$


Recall that we want to relate the equations of motion, Eq. (52), together with the Bianchi identity in Eq. (51), to the Einstein equations for the emergent metric (89). For later use, let us also introduce a completely antisymmetric tensor defined by

$$
\Psi_{a b c} \equiv \mathbf{f}_{a b c}+\mathbf{f}_{b c a}+\mathbf{f}_{c a b} \equiv \varepsilon_{a b c d} \Psi_{d}
$$

Using the decomposition in Eq. (116), one can easily see that

$$
\Psi_{a}=-\frac{1}{3 !} \varepsilon_{a b c d} \Psi_{b c d}=-\left(\mathbf{f}_{b}^{(+) i} \eta_{a b}^{i}-\mathbf{f}_{b}^{(-) i} \bar{\eta}_{a b}^{i}\right)
$$

while Eq. (97) leads to

$$
\rho_{a}=\mathbf{f}_{b a b}=-\left(\mathbf{f}_{b}^{(+) i} \eta_{a b}^{i}+\mathbf{f}_{b}^{(-) i} \bar{\eta}_{a b}^{i}\right)
$$

Note that the right-hand side of Eq. (115) is purely interaction terms between the self-dual and the anti-self-dual parts in Eq. (116). Therefore, if gauge fields satisfy the self-duality equation, Eq. (92), i.e., $\mathbf{f}_{a}^{( \pm) i}=0$, they describe a Ricci-flat manifold, i.e., $R_{a b}=0$. Of course, this result is completely consistent with the previous self-dual case.

The next step is to calculate the Einstein tensor to identify the form of the energy-momentum tensor

$$
\begin{aligned}
E_{a b}= & R_{a b}-\frac{1}{2} \delta_{a b} R \\
= & -\frac{1}{\lambda^{2}}\left(\mathbf{f}_{d}^{(+) i} \eta_{a c}^{i} \mathbf{f}_{d}^{(-) j} \bar{\eta}_{b c}^{j}+\mathbf{f}_{d}^{(+) i} \eta_{b c}^{i} \mathbf{f}_{d}^{(-) j} \bar{\eta}_{a c}^{j}\right) \\
& +\frac{1}{\lambda^{2}}\left(\mathbf{f}_{c}^{(+) i} \eta_{a c}^{i} \mathbf{f}_{d}^{(-) j} \bar{\eta}_{b d}^{j}+\mathbf{f}_{c}^{(+) i} \eta_{b c}^{i} \mathbf{f}_{d}^{(-) j} \bar{\eta}_{a d}^{j}-\delta_{a b} \mathbf{f}_{d}^{(+) i} \eta_{c d}^{i} \mathbf{f}_{e}^{(-) j} \bar{\eta}_{c e}^{j}\right)
\end{aligned}
$$

where the Ricci scalar $R$ is given by

$$
R=\frac{2}{\lambda^{2}} \mathbf{f}_{b}^{(+) i} \eta_{a b}^{i} \mathbf{f}_{c}^{(-) j} \bar{\eta}_{a c}^{j}
$$

We have adopted the conventional view that the gravitational field is represented by the spacetime metric itself. The problem, thus, reduces to finding field equations to relate the metric in Eq. (89) to the energy-momentum distribution. According to our scheme, Eq. (121), therefore, corresponds to such field equations, i.e., the Einstein equations. First, notice that the right-hand side of Eq. (121) identically vanishes for self-dual gauge fields, satisfying $\mathbf{f}_{a}^{( \pm) i}=0$, whose energy-momentum tensor also identically vanishes because their action is topological, i.e., metric independent. Howecer, for general gauge fields for which $\mathbf{f}_{a}^{( \pm) i} \neq 0$, the right-hand side of Eq. 
(121) no longer vanishes, which, in turn, enforces $E_{a b}=R_{a b}-\frac{1}{2} \delta_{a b} R \neq 0$. Because the Einstein tensor $E_{a b}$ equals some energy-momentum tensor for matter fields, the non-vanishing Einstein tensor implies that there is a nontrivial energy-momentum tensor coming from $U(1)$ gauge fields. In other words, the presence of $U(1)$ gauge fields on a symplectic spacetime not only deforms spacetime geometry according to the correspondence in Eq. (12) but also plays a role of matter fields contributing to the energy-momentum tensor. Indeed, this should be the case because the action in Eq. (44) reduces to the ordinary Maxwell theory in the commutative limit and thus has a nontrivial energy-momentum tensor. Therefore, it is natural to identify the right-hand side of Eq. (121) with some energy-momentum tensor determined by $U(1)$ gauge fields.

We intentionally make the following separation into two kinds of energy-momentum tensors denoted by $T_{a b}^{(M)}$ and $T_{a b}^{(L)}$ :

$$
\begin{aligned}
\frac{8 \pi G}{c^{4}} T_{a b}^{(M)} & =-\frac{1}{\lambda^{2}}\left(\mathbf{f}_{d}^{(+) i} \eta_{a c}^{i} \mathbf{f}_{d}^{(-) j} \bar{\eta}_{b c}^{j}+\mathbf{f}_{d}^{(+) i} \eta_{b c}^{i} \mathbf{f}_{d}^{(-) j} \bar{\eta}_{a c}^{j}\right) \\
& =-\frac{1}{\lambda^{2}} \mathbf{f}_{d}^{(+) i} \mathbf{f}_{d}^{(-) j}\left(\eta_{a c}^{i} \bar{\eta}_{b c}^{j}+\eta_{b c}^{i} \bar{\eta}_{a c}^{j}\right) \\
& =-\frac{1}{\lambda^{2}}\left(\mathbf{f}_{a c d} \mathbf{f}_{b c d}-\frac{1}{4} \delta_{a b} \mathbf{f}_{c d e} \mathbf{f}_{c d e}\right) \\
\frac{8 \pi G}{c^{4}} T_{a b}^{(L)} & =\frac{1}{\lambda^{2}}\left(\mathbf{f}_{c}^{(+) i} \eta_{a c}^{i} \mathbf{f}_{d}^{(-) j} \bar{\eta}_{b d}^{j}+\mathbf{f}_{c}^{(+) i} \eta_{b c}^{i} \mathbf{f}_{d}^{(-) j} \bar{\eta}_{a d}^{j}-\delta_{a b} \mathbf{f}_{d}^{(+) i} \eta_{c d}^{i} \mathbf{f}_{e}^{(-) j} \bar{\eta}_{c e}^{j}\right) \\
& =\frac{1}{\lambda^{2}} \mathbf{f}_{c}^{(+) i} \mathbf{f}_{d}^{(-) j}\left(\eta_{a c}^{i} \bar{\eta}_{b d}^{j}+\eta_{b c}^{i} \bar{\eta}_{a d}^{j}-\delta_{a b} \eta_{e c}^{i} \bar{\eta}_{e d}^{j}\right) \\
& =\frac{1}{2 \lambda^{2}}\left(\rho_{a} \rho_{b}-\Psi_{a} \Psi_{b}-\frac{1}{2} \delta_{a b}\left(\rho_{c}^{2}-\Psi_{c}^{2}\right)\right)
\end{aligned}
$$

where we have used the decomposition in Eq. (117) and the relations

$$
\mathbf{f}_{b}^{(+) i} \eta_{a b}^{i}=-\frac{1}{2}\left(\rho_{a}+\Psi_{a}\right), \quad \mathbf{f}_{b}^{(-) i} \bar{\eta}_{a b}^{i}=-\frac{1}{2}\left(\rho_{a}-\Psi_{a}\right)
$$

With this notation, the Einstein equations, Eq. (121), can be written as

$$
\begin{aligned}
E_{a b} & =R_{a b}-\frac{1}{2} \delta_{a b} R \\
& =\frac{8 \pi G}{c^{4}}\left(T_{a b}^{(M)}+T_{a b}^{(L)}\right) .
\end{aligned}
$$

The main motivation of the above separation was the fact that the energy-momentum tensor $T_{a b}^{(M)}$ is traceless, i.e., $T_{a a}^{(M)}=0$, because of the property $\eta_{a b}^{( \pm) i} \eta_{a b}^{(\mp) j}=0$, so the Ricci scalar in Eq. (122) is determined by the second energy-momentum tensor $T_{a b}^{(L)}$ only.

First, let us identify the real character of the energy-momentum tensor in Eq. (123). When one stares at the energy-momentum tensor in Eq. (123), one may find that it is very reminiscent of the 
Maxwell energy-momentum tensor given by

$$
T_{a b}^{(e m)}=\frac{\hbar^{2} c^{2}}{g_{Y M}^{2}}\left(F_{a c} F_{b c}-\frac{1}{4} \delta_{a b} F_{c d} F_{c d}\right),
$$

which is also traceless, i.e., $T_{a a}^{(e m)}=0$. Indeed, it was argued in Ref. [7] that the energymomentum tensor in Eq. (123) can be mapped to Eq. (126) by reversely applying the map in Eq. (12), so to speak, by translating the map $\Gamma(T M) \rightarrow C^{\infty}(M)[65]$.

There is another reason the energy-momentum tensor in Eq. (123) should be mapped to Eq. (126). Consider a commutative limit in which $|\theta|^{2} \equiv \widehat{g}_{a c} \widehat{g}_{b d} \theta^{a b} \theta^{c d}=\kappa^{2}\left|\kappa B g^{-1}\right|^{2} \rightarrow 0$. In this limit, we should recover the ordinary Maxwell theory from the action in Eq. (44), which may be more obvious from the left-hand side of Eq. (36). Because the action in Eq. (44) is defined in the commutative limit, which reduces to the Maxwell theory at $|\theta|=0$, the Maxwell theory should play a role in the Einstein equation in Eq. (125). Of course, it would be most natural for it to appear on the right-hand side of the Einstein equation, Eq. (125), as an energy-momentum tensor, as we explained above.

If so, it is still necessary to understand how the gravitational constant $G$ in Eq. (125) arose from the gauge theory in Eq. (44) because it did not contain $G$ from the outset. Recall that both Eqs. (125) and (126) are valid even in $D$-dimensions. Because the energy-momentum tensor carries the physical dimension of energy density, i.e., $\left[T_{a b}^{(e m)}\right]=\frac{M L^{2} T^{-2}}{L^{D-1}}$ and $\left[R_{a b}\right]=L^{-2}$, we need some physical constant carrying the physical dimension of $M^{-1} L^{D-1} T^{-2}$ in Eq. (125). Of course, it is the Newton constant G. See Eq. (14). However, we pointed out that, if a field theory is equipped with an intrinsic length scale, which is precisely the case for the action in Eq. (44) with $L^{2}=|\theta|$, the gravitational constant $G$ can arise purely from the field theory. In our case, this means [7] that the gravitational constant $G$ can be determined from only the field theory parameters in Eq. (44):

$$
\frac{G \hbar^{2}}{c^{2}} \sim g_{Y M}^{2}|\theta|
$$

We will wait to Section V to pose an important question on what the physical implications of Eq. (127) are because we are not yet prepared for that question.

Now, it is in order to ask about the real character of the energy-momentum tensor in Eq. (124). As we pointed out before, $T_{a b}^{(M)}$ in Eq. (123) is traceless, so the Ricci scalar in Eq. (122) should genuinely be determined by the second energy-momentum tensor in Eq. (124). For example, let us consider a maximally symmetric space in which the curvature and the Ricci tensors are given 
by

$$
R_{a b c d}=\frac{R}{D(D-1)}\left(\delta_{a c} \delta_{b d}-\delta_{a d} \delta_{b c}\right), \quad R_{a b}=\frac{R}{D} \delta_{a b} .
$$

Then, let us simply assume that the Einstein equations, Eq. (125), allow a nearly maximally symmetric spacetime. In this case, the energy-momentum tensor in Eq. (124) will be dominant, and the global structure of spactime will be determined by $T_{a b}^{(L)}$ only.

To descry closer aspects of the energy-momentum tensor in Eq. (124), let us consider the following decomposition:

$$
\begin{aligned}
& \rho_{a} \rho_{b}=\frac{1}{4} \delta_{a b} \rho_{c}^{2}+\left(\rho_{a} \rho_{b}-\frac{1}{4} \delta_{a b} \rho_{c}^{2}\right) \\
& \Psi_{a} \Psi_{b}=\frac{1}{4} \delta_{a b} \Psi_{c}^{2}+\left(\Psi_{a} \Psi_{b}-\frac{1}{4} \delta_{a b} \Psi_{c}^{2}\right) .
\end{aligned}
$$

In the above decomposition, the first terms correspond to scalar modes and will be a source of the expansion/contraction of spacetime while the second terms correspond to quadruple modes and will give rise to the shear distortion of spacetime, which can be seen via Raychauduri's equation (288). For a nearly maximally symmetric spacetime, the second terms can, thus, be neglected. In this case, the energy-momentum tensor in Eq. (124) behaves as a cosmological constant for a (nearly) constant-curvature spacetime, i.e.,

$$
T_{a b}^{(L)}=-\frac{c^{4} R}{32 \pi G} \delta_{a b}
$$

In Section V, we will consider the Wick rotation, $y^{4}=i y^{0}$, of the energy-momentum tensor in Eq. (124) and discuss a very surprising aspect of it in Minkowski spacetime.

\section{QUANTUM GRAVITY}

Riemannian geometry has been charged with a primary role in describing the theory of gravity, but many astronomical phenomena involved with very strongly gravitating systems, e.g., the Big-Bang, black holes, etc., disclose that a Riemannian geometry describing a smooth spacetime manifold is not enough. Instead, it turns out that a "quantum geometry" is necessary to describe such extremely gravitating systems. Unfortunately, we still do not know how to quantize a Riemannian manifold in order to define the quantum geometry.

In the previous section, we showed that a Riemannian geometry could emerge from a Poisson geometry in the context of emergent gravity. The underlying Poisson geometry has been defined 
by a $U(1)$ gauge theory on a Poisson spacetime. Therefore, we may quantize the Poisson geometry to define quantum gravity. Now, we want to explore how the Poisson geometry defined by the $U(1)$ gauge theory on the Poisson spacetime can be quantized to describe quantum geometries.

\section{A. Quantum Equivalence Principle}

In Section I, we have suggested that the quantization of gravity might be defined by the spacetime deformation in terms of $G$ rather than $\hbar$. If spin-two graviton were really a fundamental particle, it could be physically viable to quantize gravity in terms of the Planck constant $\hbar$ which will quantize the particle phase space of gravitons. However, recent developments in string theory [11], known as the AdS/CFT duality, open-closed string duality, matrix models, etc., imply that gravity may be a collective phenomenon emergent from gauge fields. That is, the spin-two graviton might arise as a composite of two spin-one gauge bosons. Presumably, this composite nature of gravitons is already immanent in the vielbein formalism as the metric expression in Eq. (2) politely insinuates.

In Section II, we showed that Einstein gravity can be formulated in terms of a symplectic geometry rather than a Riemannian geometry in the context of emergent gravity. An essential step for emergent gravity was to realize the equivalence principle, the most important property in the theory of gravity (general relativity), from $U(1)$ gauge theory on a symplectic or a Poisson manifold. Through the realization of the equivalence principle, which is an intrinsic property in symplectic geometry [8], known as the Darboux theorem or the Moser lemma, we can understand how diffeomorphism symmetry arises from symplectic $U(1)$ gauge theory and how gravity can emerge from symplectic electromagnetism, which is also an interacting theory.

A unique feature of gravity disparate from other physical interactions is that it is characterized by the Newton constant $G$ whose physical dimension is (length) $)^{2}$ in natural units. We have to deeply ruminate about its physical origin. Our proposal is that it is inherited from a Poisson structure of spacetime. In order to support that, we have elucidated how gravity can emerge from a field theory on such a spacetime. Also, we have realized such an idea in Eq. (127) that the gravitational constant $G$ can be purely determined by the gauge theory parameters, signaling the emergence of gravity from the field theory. Remarkably, it turns out that $U(1)$ gauge theory defined with an intrinsic length scale set by the Poisson structure in Eq. (6) should be a theory of gravity.

Therefore, it is now obvious how to quantize gravity if gravity is emergent from a gauge theory 
defined on a symplectic or Poisson manifold. We already briefly speculated in subsection I.C how quantum geometry can arise from the quantization of spacetime, i.e., a noncommutative spacetime. We will clarify more how the essential properties of emergent gravity can be lifted to the noncommutative spacetime. In particular, we want to clarify how the Darboux theorem, as the equivalence principle for emergent gravity, can be realized in a full noncommutative geometry. It was already convincingly argued in Ref. [5] that such a kind of "quantum equivalence principle" exists in the context of deformation quantization $\grave{a}$ la Kontsevich as a gauge equivalence between star products. Actually, this gauge equivalence between star products reduces in the commutative limit to the usual Darboux theorem and was the basis of the Seiberg-Witten map between commutative and noncommutative gauge fields [16], as was also discussed in Ref. [5]. Therefore, a general noncommutative deformation of emergent gravity would be possible because Kontsevich already proved [15] that any Poisson manifold can always be quantized at least in the context of deformation quantization.

As we argued in Section II, the Darboux theorem, or more precisely the Moser lemma, in symplectic geometry is enough to derive Einstein gravity because the latter arises from a $U(1)$ gauge theory on a symplectic manifold. Now, we want to quantize the $U(1)$ gauge theory defined by the action in Eq. (44) à la Dirac, i.e., by adopting the quantization rule in Eq. (7):

$$
\begin{aligned}
& D_{a} \in C^{\infty}(M) \rightarrow \widehat{D}_{a}=B_{a b} y^{b}+\widehat{A}_{a} \in \mathcal{A}_{\theta}, \\
& \left\{D_{a}, D_{b}\right\}_{\theta} \rightarrow-i\left[\widehat{D}_{a}, \widehat{D}_{b}\right] \equiv-B_{a b}+\widehat{F}_{a b},
\end{aligned}
$$

where $\widehat{F}_{a b} \in \mathcal{A}_{\theta}$ is the noncommutative field strength defined by

$$
\widehat{F}_{a b}=\partial_{a} \widehat{A}_{b}-\partial_{b} \widehat{A}_{a}-i\left[\widehat{A}_{a}, \widehat{A}_{b}\right]
$$

Here, we understand the noncommutative fields in Eq. (131) as being self-adjoint operators acting on the Hilbert space $\mathcal{H}$. Then, we get the $U(1)$ gauge theory defined on the noncommutative spacetime in Eq. (8) as

$$
\widehat{S}=-\frac{1}{4 G_{s}} \operatorname{Tr} \mathcal{H}\left[\widehat{D}_{a}, \widehat{D}_{b}\right]\left[\widehat{D}^{a}, \widehat{D}^{b}\right]
$$

where $G_{s} \equiv g_{s} / 2 \pi \kappa^{2}$ and the trace $\operatorname{Tr}_{\mathcal{H}}$ is defined over the Fock space in Eq. (18) and can be identified with

$$
\operatorname{Tr}_{\mathcal{H}} \equiv \int \frac{d^{2 n} y}{(2 \pi)^{n}|\operatorname{Pf} \theta|} .
$$


The Jacobi identity for the operator algebra $\mathcal{A}_{\theta}$ leads to the Bianchi identity

$$
\left[\widehat{D}_{[a},\left[\widehat{D}_{b}, \widehat{D}_{c]}\right]\right]=-\widehat{D}_{[a} \widehat{F}_{b c]}=0
$$

and the equations of motion derived from the action in Eq. (133) read as

$$
\left[\widehat{D}^{a},\left[\widehat{D}_{a}, \widehat{D}_{b}\right]\right]=-\widehat{D}^{a} \widehat{F}_{a b}=0
$$

where

$$
\widehat{D}_{a} \widehat{F}_{b c}=\partial_{a} \widehat{F}_{b c}-i\left[\widehat{A}_{a}, \widehat{F}_{b c}\right]
$$

In classical mechanics, the set of possible states of a particle system forms a Poisson manifold $P$. The observables that we want to measure are smooth functions in $C^{\infty}(P)$, forming a commutative (Poisson) algebra. In quantum mechanics, the set of possible states is represented by a Hilbert space $\mathcal{H}$. The observables are self-adjoint operators acting on $\mathcal{H}$, forming a noncommutative $\star$-algebra. The change from a Poisson manifold to a Hilbert space is a pretty big one.

A natural question is whether a quantization, such as Eq. (7), for a spacetime manifold $M$ with a general Poisson structure $\pi=\frac{1}{2} \pi^{\mu \nu}(x) \frac{\partial}{\partial x^{\mu}} \wedge \frac{\partial}{\partial x^{\nu}} \in \Gamma\left(\Lambda^{2} T M\right)$ is always possible with a radical change in the nature of the observables. The problem is how to construct the Hilbert space for a general Poisson manifold, which is, in general, highly nontrivial. Deformation quantization was proposed in Ref. [66] as an alternative, where the quantization is understood to be a deformation of the algebra $\mathcal{A}=C^{\infty}(M)$ of classical observables. Instead of building a Hilbert space from a Poisson manifold and associating an algebra of operators to it, we are only concerned with the algebra $\mathcal{A}$ to deform the commutative product in $C^{\infty}(M)$ to a noncommutative and associative product. In a canonical phase space where $\pi=\theta$ such as the case we have considered so far, it is easy to show that the two approaches have a one-to-one correspondence through the Weyl-Moyal $\operatorname{map}[9,10]$ :

$$
\widehat{f} \cdot \widehat{g} \cong(f \star g)(y)=\left.\exp \left(\frac{i}{2} \theta^{\mu \nu} \partial_{\mu}^{y} \partial_{\nu}^{z}\right) f(y) g(z)\right|_{y=z} .
$$

Recently Kontsevich answered the above question in the context of deformation quantization [15]. He proved that every finite-dimensional Poisson manifold $M$ admits a canonical deformation quantization and that changing coordinates leads to gauge-equivalent star products. We briefly recapitulate his results, which will be useful for our later discussions. Let $\mathcal{A}$ be the algebra over $\mathbb{R}$ 
of smooth functions on a finite-dimensional $C^{\infty}$-manifold $M$. A star product on $\mathcal{A}$ is an associative $\mathbb{R}[[\hbar]]$-bilinear product on the algebra $\mathcal{A}[[\hbar]]$, a formal power series in $\hbar$ with coefficients in $C^{\infty}(M)=\mathcal{A}$, given by the following formula for $f, g \in \mathcal{A} \subset \mathcal{A}[[\hbar]][67]$ :

$$
(f, g) \mapsto f \star g=f g+\hbar B_{1}(f, g)+\hbar^{2} B_{2}(f, g)+\cdots \in \mathcal{A}[[\hbar]]
$$

where $B_{i}(f, g)$ are bidifferential operators. There is a natural gauge group which acts on star products. This group consists of automorphisms of $\mathcal{A}[[\hbar]]$ considered as an $\mathbb{R}[[\hbar]]$-module (i.e., linear transformations $\mathcal{A} \rightarrow \mathcal{A}$ parameterized by $\hbar$ ). If $D(\hbar)=1+\sum_{n \geq 1} \hbar^{n} D_{n}$ is such an automorphism, where $D_{n}: \mathcal{A} \rightarrow \mathcal{A}$ are differential operators, it acts on the set of star products as

$$
\star \rightarrow \star^{\prime}, \quad f(\hbar) \star^{\prime} g(\hbar)=D(\hbar)\left(D(\hbar)^{-1}(f(\hbar)) \star D(\hbar)^{-1}(g(\hbar))\right)
$$

for $f(\hbar), g(\hbar) \in \mathcal{A}[[\hbar]]$. This is evident from the commutativity of the diagram



Two star products $\star$ and $\star^{\prime}$ are called equivalent if there exists an automorphism $D(\hbar)$, a formal power series of differential operators, satisfying Eq. (140). We are interested in star products up to the gauge equivalence. This equivalence relation is closely related to the cohomological Hochschild complex of algebra $\mathcal{A}$ [15], i.e., the algebra of smooth polyvector fields on $M$. For example, it follows from the associativity of the product in Eq. (139) that the symmetric part of $B_{1}$ can be killed by a gauge transformation that is a coboundary in the Hochschild complex, and that the antisymmetric part of $B_{1}$, denoted as $B_{1}^{-}$, comes from a bivector field $\pi \in \Gamma\left(\Lambda^{2} T M\right)$ on $M$ :

$$
B_{1}^{-}(f, g)=\langle\pi, d f \otimes d g\rangle
$$

In fact, any Hochschild coboundary can be removed by using a gauge transformation $D(\hbar)$, thus leading to the gauge equivalent star product in Eq. (140). The associativity at $\mathcal{O}\left(\hbar^{2}\right)$ further constrains that $\pi$ must be a Poisson structure on $M$; in other words, $[\pi, \pi]_{S N}=0$, where the bracket is the Schouten-Nijenhuis bracket on polyvector fields (see Ref. [15] for the definition of this bracket and the Hochschild cohomology). Thus, gauge equivalence classes of star products 
modulo $\mathcal{O}\left(\hbar^{2}\right)$ are classified by Poisson structures on $M$. It was shown [15] that there are no other obstructions to deforming the algebra $\mathcal{A}$ up to arbitrary higher orders in $\hbar$.

For an equivalence class of star products for any Poisson manifold, Kontsevich arrived at the following general result [15]:

The set of gauge equivalence classes of star products on a smooth manifold $M$ can be naturally identified with the set of equivalence classes of Poisson structures depending formally on $\hbar$

$$
\pi=\pi(\hbar)=\pi_{1} \hbar+\pi_{2} \hbar^{2}+\cdots \in \Gamma\left(\Lambda^{2} T M\right)[[\hbar]], \quad[\pi, \pi]_{S N}=0 \in \Gamma\left(\Lambda^{3} T M\right)[[\hbar]]
$$

modulo the action of the group of formal paths in the diffeomorphism group of $M$, starting at the identity diffeomorphism. Also, if we change coordinates in Eq. (139), we obtain a gaugeequivalent star product.

This theorem means that the set of equivalence classes of associative algebras close to algebras of functions on manifolds is in one-to-one correspondence with the set of equivalence classes of Poisson manifolds module diffeomorphisms.

Suppose that the Poisson tensor $\pi=\frac{1}{2} \pi^{\mu \nu}(x) \frac{\partial}{\partial x^{\mu}} \wedge \frac{\partial}{\partial x^{\nu}} \in \Gamma\left(\Lambda^{2} T M\right)$ is a nondegenerate constant bi-vector and denote it with $\theta$ again. In this case, the star product is given by Eq. (138), the so-called Moyal product. If we make an arbitrary change of coordinates, $y^{\mu} \mapsto x^{a}(y)$, in the Moyal $\star$-product in Eq. (138), which is nothing but the Kontsevich's star product in Eq. (139) with the constant Poisson bi-vector $\theta$, we will get a new star product, Eq. (139), defined by a Poisson bi-vector $\pi(\hbar)$. However, the resulting star product has to be gauge equivalent to the Moyal product in Eq. (138) and $\pi(\hbar)$ belongs to the same equivalence class of Poisson structures and so could be determined by using the formal power series in Eq. (142) for the original Poisson bi-vector $\theta$. Conversely, if two star products $\star$ and $\star^{\prime}$ are gauge equivalent in the sense that there exists an automorphism $D(\hbar)$ satisfying Eq. (140), the Poisson structures $\theta$ and $\pi$ defining the star products $\star$ and $\star^{\prime}$, respectively, must belong to the same gauge equivalence class. This is the general statement of the above theorem.

Actually, it is easy to show that the gauge equivalence relation in Eq. (140) between star products reduces to the Darboux transformation (29) in the commutative limit where $D(\hbar)=1$. After identifying $\pi^{-1}=B+F$ and $\theta^{-1}=B$, we get in this limit

$$
\left\{x^{a}, x^{b}\right\}_{\theta}(y)=\theta^{\mu \nu} \frac{\partial x^{a}}{\partial y^{\mu}} \frac{\partial x^{b}}{\partial y^{\nu}}=\pi^{a b}(x)=\left(\frac{1}{B+F}\right)^{a b}(x),
$$

which is precisely the inverse of Eq. (29) if $\omega_{1}=\pi^{-1}$ and $\omega_{0}=\theta^{-1}$. Therefore, we propose [5] the "quantum equivalence principle" as the gauge equivalence in Eq. (140) between star products 
in the sense that the Darboux theorem as the equivalence principle for emergent gravity is lifted to a noncommutative geometry. Furthermore, the isomorphism in Eq. (34) from the Lie algebra of Poisson vector fields to the Lie algebra of vector fields as derivations of $C^{\infty}(M)$ can be lifted to the noncommutative spacetime in Eq. (8) as follows: Consider an adjoint operation of noncommutative gauge fields $\widehat{D}_{a}(y) \in \mathcal{A}_{\theta}$ in Eq. (131),

$$
\widehat{V}_{a}[\widehat{f}](y) \equiv-i\left[\widehat{D}_{a}(y), \widehat{f}(y)\right]_{\star} .
$$

The leading term in Eq. (144) exactly recovers the vector fields in Eq. (12), i.e.,

$$
\begin{aligned}
\widehat{V}_{a}[\widehat{f}](y) & =-i\left[\widehat{D}_{a}(y), \widehat{f}(y)\right]_{\star}=-\theta^{\mu \nu} \frac{\partial D_{a}(y)}{\partial y^{\nu}} \frac{\partial f(y)}{\partial y^{\mu}}+\cdots \\
& =V_{a}[f](y)+\mathcal{O}\left(\theta^{3}\right) .
\end{aligned}
$$

Because the star product in Eq. (139) is associative, one can show the following properties:

$$
\begin{aligned}
& \widehat{V}_{a}[\widehat{f} \star \widehat{g}]=\widehat{V}_{a}[\widehat{f}] \star \widehat{g}+\widehat{f} \star \widehat{V}_{a}[\widehat{g}] \\
& \widehat{V}_{-i\left[\widehat{D}_{a}, \widehat{D}_{a}\right]_{\star}}=\left[\widehat{V}_{a}, \widehat{V}_{b}\right]_{\star} .
\end{aligned}
$$

The above property implies that we can identify the adjoint operation $\operatorname{Der}\left(\mathcal{A}_{\theta}\right) \equiv\left\{\widehat{V}_{a} \mid a=\right.$ $1, \cdots, 2 n\}$ with the (inner) derivations of a noncommutative $\star$-algebra $\mathcal{A}_{\theta}$, and so the generalization of vector fields $\Gamma(T M)=\left\{V_{a} \mid a=1, \cdots, 2 n\right\}$ in Eq. (12). Using Eq. (146), one can show that

$$
\begin{aligned}
& \widehat{V}_{\widehat{F}_{a b}}=\left[\widehat{V}_{a}, \widehat{V}_{b}\right]_{\star}, \\
& \widehat{V}_{\widehat{D}_{a} \widehat{F}_{b c}}=\left[\widehat{V}_{a},\left[\widehat{V}_{b}, \widehat{V}_{c}\right]_{\star}\right]_{\star},
\end{aligned}
$$

which may be compared with Eqs. (49) and (50). We can use the map in Eq. (148) in exactly the same way as in the Poisson algebra case to translate the Jacobi identity in Eq. (135) and the equations of motion in Eq. (136) into some relations between the generalized vector fields $\widehat{V}_{a}$ defined by Eq. (144) [7]:

$$
\begin{aligned}
& \widehat{D}_{[a} \widehat{F}_{b c]}=0 \quad \Leftrightarrow \quad\left[\widehat{V}_{[a},\left[\widehat{V}_{b}, \widehat{V}_{c]}\right]_{\star}\right]_{\star}=0, \\
& \widehat{D}^{a} \widehat{F}_{a b}=0 \quad \Leftrightarrow \quad\left[\widehat{V}^{a},\left[\widehat{V}_{a}, \widehat{V}_{b}\right]_{\star}\right]_{\star}=0 .
\end{aligned}
$$

We will consider the system of the derivations of noncommutative $\star$-algebra $\mathcal{A}_{\theta}$ defined by Eqs. (149) and (150) as the quantization of the system given by Eqs. (51) and (52) and, thus, as quantization of Einstein gravity in the sense of Eq. (7). To support the claim, we will take 
the correspondence in Eq. (22) to show [6] that any large- $N$ gauge theory can be mapped to a noncommutative $U(1)$ gauge theory like as Eq. (133). Because the large- $N$ gauge theory is believed to provide a theory of quantum geometries as evidenced by the AdS/CFT correspondence and various matrix models in string theory, we think it could be reasonable evidence for our claim.

\section{B. Noncommutative Electromagnetism as a Large- $N$ Gauge Theory}

Let us consider $U(N \rightarrow \infty)$ Yang-Mills theory in $d$ dimensions:

$$
S_{M}=-\frac{1}{G_{s}} \int d^{d} z \operatorname{Tr}\left(\frac{1}{4} F_{\mu \nu} F^{\mu \nu}+\frac{1}{2} D_{\mu} \Phi^{a} D^{\mu} \Phi^{a}-\frac{1}{4}\left[\Phi^{a}, \Phi^{b}\right]^{2}\right)
$$

where $G_{s} \equiv 2 \pi g_{s} /(2 \pi \kappa)^{\frac{4-d}{2}}$ and $\Phi^{a}(a=1, \cdots, 2 n)$ are adjoint scalar fields in $U(N)$. Here, the $d$-dimensional commutative spacetime $\mathbb{R}_{C}^{d}$ will be taken with either a Lorentzian or a Euclidean signature. Note that, if $d=4$ and $n=3$, the action in Eq. (151) is exactly the bosonic part of the 4-dimensional $\mathcal{N}=4$ supersymmetric $U(N)$ Yang-Mills theory, which is the large- $N$ gauge theory of the AdS/CFT correspondence.

Suppose that a vacuum of the theory, Eq. (151), is given by

$$
\left\langle\Phi^{a}\right\rangle_{\mathrm{vac}}=\frac{1}{\kappa} y^{a}, \quad\left\langle A_{\mu}\right\rangle_{\mathrm{vac}}=0 .
$$

We will assume that the vacuum expectation values $y^{a} \in U(N)$ in the $N \rightarrow \infty$ limit satisfy the algebra

$$
\left[y^{a}, y^{b}\right]=i \theta^{a b} \mathbf{1}_{N \times N},
$$

where $\theta^{a b}$ is a constant matrix of rank $2 n$. If so, the vacuum in Eq. (152) is definitely a solution to the theory in Eq. (151) and the large- $N$ matrices $y^{a}$ can be mapped to noncommutative fields according to the correspondence in Eq. (22). The adjoint scalar fields in vacuum then satisfy the noncommutative Moyal algebra defined by Eq. (8) or equivalently

$$
\left[y^{a}, y^{b}\right]_{\star}=i \theta^{a b}
$$

Now, let us expand the large- $N$ matrices in the action in Eq. (151) around the vacuum in Eq. (152):

$$
\begin{aligned}
\Phi^{a}(z, y) & =\frac{1}{\kappa}\left(y^{a}+\theta^{a b} \widehat{A}_{b}(z, y)\right), \\
D_{\mu}(z, y) & =\partial_{\mu}-i \widehat{A}_{\mu}(z, y),
\end{aligned}
$$


where we have assumed that the fluctuations $\widehat{A}_{M}(X) \equiv\left(\widehat{A}_{\mu}, \widehat{A}_{a}\right)(z, y), M=1, \cdots, d+2 n$, also depend on the vacuum moduli in Eq. (152). Therefore, let us introduce $D=d+2 n$-dimensional coordinates $X^{M}=\left(z^{\mu}, y^{a}\right)$, which consist of $d$-dimensional commutative ones denoted by $z^{\mu}(\mu=1, \cdots, d)$ and $2 n$-dimensional noncommutative ones denoted by $y^{a}(a=1, \cdots, 2 n)$, satisfying the relation in Eq. (154). Likewise, $D$-dimensional gauge fields $\widehat{A}_{M}(X)$ are also introduced in a similar way [68]:

$$
\begin{aligned}
D_{M}(X) & =\partial_{M}-i \widehat{A}_{M}(X) \\
& \equiv\left(D_{\mu}=\partial_{\mu}-i \widehat{A}_{\mu}, D_{a}=-i \kappa B_{a b} \Phi^{b}\right)(z, y)
\end{aligned}
$$

According to the correspondence in Eq. (22), we will replace the matrix commutator in the action in Eq. (151) by the star commutator, i.e.,

$$
[\mathbf{Q}, \mathbf{M}]_{N \times N} \quad \rightarrow \quad[\mathbf{c}, \mathbf{M}]_{\star} .
$$

It is then straightforward to calculate each component in the matrix action in Eq. (151)

$$
\begin{aligned}
& F_{\mu \nu}=i\left[D_{\mu}, D_{\nu}\right]_{\star}=\partial_{\mu} \widehat{A}_{\nu}-\partial_{\nu} \widehat{A}_{\mu}-i\left[\widehat{A}_{\mu}, \widehat{A}_{\nu}\right]_{\star}:=\widehat{F}_{\mu \nu}, \\
& D_{\mu} \Phi^{a}=i \frac{\theta^{a b}}{\kappa}\left[D_{\mu}, D_{b}\right]_{\star}=\frac{\theta^{a b}}{\kappa}\left(\partial_{\mu} \widehat{A}_{b}-\partial_{b} \widehat{A}_{\mu}-i\left[\widehat{A}_{\mu}, \widehat{A}_{b}\right]_{\star}\right):=\frac{\theta^{a b}}{\kappa} \widehat{F}_{\mu b}, \\
& {\left[\Phi^{a}, \Phi^{b}\right]=-\frac{1}{\kappa^{2}} \theta^{a c} \theta^{b d}\left[D_{c}, D_{d}\right]_{\star}=\frac{i}{\kappa^{2}} \theta^{a c} \theta^{b d}\left(-B_{c d}+\partial_{c} \widehat{A}_{d}-\partial_{d} \widehat{A}_{c}-i\left[\widehat{A}_{c}, \widehat{A}_{d}\right]_{\star}\right)} \\
& :=-\frac{i}{\kappa^{2}}(\theta(\widehat{F}-B) \theta)^{a b},
\end{aligned}
$$

where we defined $\left[\partial_{\mu}, \widehat{f}\right]_{\star}=\partial_{\mu} \widehat{f}$ and $B=\frac{1}{2} B_{a b} d y^{a} \wedge d y^{b}$ with $\operatorname{rank}(B)=2 n$. It is important to notice that large- $N$ matrices on the vacuum in Eq. (152) are now represented by their master fields, which are higher-dimensional noncommutative $U(1)$ gauge fields in Eq. (157) whose field strength is given by

$$
\widehat{F}_{M N}=\partial_{M} \widehat{A}_{N}-\partial_{N} \widehat{A}_{M}-i\left[\widehat{A}_{M}, \widehat{A}_{N}\right]_{\star}
$$

Collecting all the results in Eq. (159) and using the trace in Eq. (134), the action in Eq. (151) can be recast into the simple form [6]

$$
\widehat{S}_{B}=-\frac{1}{4 g_{Y M}^{2}} \int d^{D} X \sqrt{-G} G^{M P} G^{N Q}\left(\widehat{F}_{M N}-B_{M N}\right) \star\left(\widehat{F}_{P Q}-B_{P Q}\right),
$$

where we have assumed a constant metric on $\mathbb{R}^{D}=\mathbb{R}_{C}^{d} \times \mathbb{R}_{N C}^{2 n}$ with the form

$$
\begin{aligned}
d s^{2} & =G_{M N} d X^{M} d X^{N} \\
& =g_{\mu \nu} d z^{\mu} d z^{\nu}+\widehat{g}_{a b} d y^{a} d y^{b}
\end{aligned}
$$


and the relations in Eqs. (38), (39) and (40) were used. In the end, the $d$-dimensional $U(N)$ Yang-Mills theory in Eq. (151) has been transformed into a $D$-dimensional noncommutative $U(1)$ gauge theory.

Depending on the choice of the base space $\mathbb{R}_{C}^{d}$, one can get a series of matrix models from the large- $N$ gauge theory in Eq. (151): for instance, the IKKT matrix model for $d=0$ [18], the BFSS matrix model for $d=1$ [17] and the matrix string theory for $d=2$ [19]. The most interesting case is $d=4$ and $n=3$, which is equal to the bosonic part of the 4-dimensional $\mathcal{N}=4$ supersymmetric $U(N)$ Yang-Mills theory in the AdS/CFT duality [22] and is equivalent to the 10-dimensional noncommutative $U(1)$ gauge theory on $\mathbb{R}_{C}^{4} \times \mathbb{R}_{N C}^{6}$. Note that all these matrix models or large- $N$ gauge theories are nonperturbative formulations of string or $\mathrm{M}$ theories. Therefore, it should be reasonable to expect that the $d$-dimensional $U(N \rightarrow \infty)$ gauge theory in Eq. (151) and so the $D$-dimensional noncommutative $U(1)$ gauge theory in Eq. (161) describe a theory of quantum gravity according to the large- $N$ duality or AdS/CFT correspondence.

We will give further evidences why the matrix action in Eq. (151) contains a variety of quantum geometries and how smooth Riemannian geometries can be emergent from the action in Eq. (161) in a commutative limit. First, apply the adjoint operation in Eq. (144) to the $D$-dimensional noncommutative gauge fields $D_{A}(X)=\left(D_{\mu}, D_{a}\right)(z, y)$ (after switching the index $M \rightarrow A$ to distinguish them from the local coordinate indices $M, N, \cdots)$ to obtain

$$
\begin{aligned}
\widehat{V}_{A}[\widehat{f}](X) & =\left[D_{A}, \widehat{f}\right]_{\star}(z, y) \\
& \equiv V_{A}^{M}(z, y) \partial_{M} f(z, y)+\mathcal{O}\left(\theta^{3}\right),
\end{aligned}
$$

where $V_{A}^{\mu}=\delta_{A}^{\mu}$ because the star product acts only on $y$-coordinates and we define $\left[\partial_{\mu}, \widehat{f}(X)\right]_{\star}=$ $\frac{\partial \widehat{f}(X)}{\partial z^{\mu}}$. More explicitly, the $D$-dimensional noncommutative $U(1)$ gauge fields at leading order appear as the usual vector fields (frames on a tangent bundle) on a $D$-dimensional manifold $M$ given by

$$
V_{A}(X)=\left(\partial_{\mu}+A_{\mu}^{a} \partial_{a}, D_{a}^{b} \partial_{b}\right)
$$

or with matrix notation

$$
V_{A}^{M}(X)=\left(\begin{array}{cc}
\delta_{\mu}^{\nu} & A_{\mu}^{a} \\
0 & D_{a}^{b}
\end{array}\right),
$$

where

$$
A_{\mu}^{a} \equiv-\theta^{a b} \frac{\partial \widehat{A}_{\mu}}{\partial y^{b}}, \quad D_{a}^{b} \equiv \delta_{a}^{b}-\theta^{b c} \frac{\partial \widehat{A}_{a}}{\partial y^{c}} .
$$


One can easily check that $V_{A}$ 's in Eq. (164) take values in the Lie algebra of volume-preserving vector fields, i.e., $\partial_{M} V_{A}^{M}=0$. One can also determine the dual basis $V^{A}=V_{M}^{A} d X^{M} \in \Gamma\left(T^{*} M\right)$, i.e., $\left\langle V^{A}, V_{B}\right\rangle=\delta_{B}^{A}$, which is given by

$$
V^{A}(X)=\left(d z^{\mu}, V_{b}^{a}\left(d y^{b}-A_{\mu}^{b} d z^{\mu}\right)\right)
$$

or with matrix notation

$$
V_{M}^{A}(X)=\left(\begin{array}{cc}
\delta_{\mu}^{\nu} & -V_{b}^{a} A_{\mu}^{b} \\
0 & V_{b}^{a}
\end{array}\right),
$$

where $V_{a}^{c} D_{c}^{b}=\delta_{a}^{b}$.

From the previous analysis in Section II.C (which corresponds to the $d=0$ case), we know that the vector fields $V_{A}$ determined by gauge fields are related to the orthonormal frames (vielbeins) $E_{A}$ by $V_{A}=\lambda E_{A}$ and $E^{A}=\lambda V^{A}$, where the conformal factor $\lambda$ will be determined later. (This situation is very reminiscent of the string frame $\left(V_{A}\right)$ and the Einstein frame $\left(E_{A}\right)$ in string theory.) Hence, the $D$-dimensional metric can be determined explicitly, by using the dual basis (167) up to a conformal factor [69]:

$$
\begin{aligned}
d s^{2} & =\eta_{A B} E^{A} \otimes E^{B} \\
& =\lambda^{2} \eta_{A B} V^{A} \otimes V^{B}=\lambda^{2} \eta_{A B} V_{M}^{A} V_{N}^{B} d X^{M} \otimes d X^{N} \\
& =\lambda^{2}\left(\eta_{\mu \nu} d z^{\mu} d z^{\nu}+\delta_{a b} V_{c}^{a} V_{d}^{b}\left(d y^{c}-\mathbf{A}^{c}\right)\left(d y^{d}-\mathbf{A}^{d}\right)\right)
\end{aligned}
$$

where $\mathbf{A}^{a}=A_{\mu}^{a} d z^{\mu}$.

The conformal factor $\lambda^{2}$ in the metric in Eq. (169) can be determined in exactly the same way as in the Section II.C. Choose a $D$-dimensional volume form with a matching parameter $\lambda \in C^{\infty}(M)$ such that

$$
\nu=\lambda^{2} V^{1} \wedge \cdots \wedge V^{D}
$$

and

$$
\lambda^{2}=\nu\left(V_{1}, \cdots, V_{D}\right)
$$

Then, the vector fields $V_{A}$ are volume preserving with respect to a $D$-dimensional volume form $\nu=\lambda^{(2-D)} \nu_{g}$, where

$$
\nu_{g}=E^{1} \wedge \cdots \wedge E^{D}
$$


and the vector fields $E_{A}$ are volume preserving with respect to another volume form $\widetilde{\nu}=\lambda^{(3-D)} \nu_{g}$. Because $\partial_{M} V_{A}^{M}=0$ or $\mathcal{L}_{V_{A}} \nu=0$, we can choose the invariant volume by turning off all fluctuations in Eq. (170) as

$$
\nu=d z^{1} \wedge \cdots \wedge d z^{d} \wedge d y^{1} \wedge \cdots \wedge d y^{2 n}
$$

Then, we finally get

$$
\lambda^{2}=\operatorname{det}^{-1} V_{b}^{a}
$$

One can see that the spacetime geometry described by the metric in Eq. (169) is completely determined by noncommutative gauge fields whose underlying theory is defined by the action in Eq. (151) or in Eq. (161). One may also confirm the claim in Section I.A that a spin-two graviton arises as a composite of two spin-one vector fields and such spin-one vector fields arise from electromagnetic fields living in the noncommutative spacetime of Eq. (154). However, one has to remember that the spacetime geometry in Eq. (169) is responsible only at the leading order, i.e., $\mathcal{O}(\theta)$, of the generalized vector fields defined by Eq. (163). All higher derivative terms in the star product in Eq. (163) are simply ignored. If such higher derivative terms are included in the star product in Eq. (163) order by order, they will deform the Einstein gravity order by order as a response to the noncommutative effects of spacetime. (See Ref. [5] for higher-order corrections to emergent gravity.) If a probe goes into a deep microscopic world where the noncommutative effect of spacetime will grow significantly, the gravity description in Eq. (169) in terms of smooth geometries will gradually become crude and coarse. In the deep noncommutative space, we have to replace Einstein gravity by a more fundamental theory describing quantum gravity or a noncommutative geometry. We argued that such a fundamental theory could be implemented by using the large- $N$ gauge theory in Eq. (151) or the higher-dimensional noncommutative $U(1)$ gauge theory 161). First note that

$$
\begin{aligned}
& {\left[D_{A}, D_{B}\right]_{\star}=-i\left(\widehat{F}_{A B}-B_{A B}\right),} \\
& {\left[D_{A},\left[D_{B}, D_{C}\right]_{\star}\right]_{\star}=-i \widehat{D}_{A} \widehat{F}_{B C},}
\end{aligned}
$$

where

$$
\widehat{D}_{A} \widehat{F}_{B C} \equiv \partial_{A} \widehat{F}_{B C}-i\left[\widehat{D}_{A}, \widehat{F}_{B C}\right]_{\star}
$$


Therefore, the Bianchi identity and the equations of motion for the action in Eq. (161) can be written as

$$
\begin{aligned}
& \widehat{D}_{[A} \widehat{F}_{B C]}=i\left[D_{[A},\left[D_{B}, D_{C]}\right]_{\star}\right]_{\star}=0, \\
& \widehat{D}^{A} \widehat{F}_{A B}=i\left[D^{A},\left[D_{A}, D_{B}\right]_{\star}\right]_{\star}=0 .
\end{aligned}
$$

Then, the above equations can be translated into some "geometric" equations of generalized vector fields defined in Eq. (163):

$$
\begin{array}{lll}
\widehat{D}_{[A} \widehat{F}_{B C]}=0 & \Leftrightarrow & {\left[\widehat{V}_{[A},\left[\widehat{V}_{B}, \widehat{V}_{C]}\right]_{\star}\right]_{\star}=0,} \\
\widehat{D}^{A} \widehat{F}_{A B}=0 & \Leftrightarrow & {\left[\widehat{V}^{A},\left[\widehat{V}_{A}, \widehat{V}_{B}\right]_{\star}\right]_{\star}=0 .}
\end{array}
$$

It may be useful to introduce a noncommutative version of the structure equation, Eq. (90):

$$
\left[\widehat{V}_{A}, \widehat{V}_{B}\right]_{\star}=-\widehat{\mathfrak{F}}_{A B}{ }^{C} \widehat{V}_{C}
$$

with the ordering prescription that the structure coefficients $\widehat{\mathfrak{F}}_{A B}{ }^{C} \in \mathcal{A}_{\theta}$ are always coming to the left-hand side. Equations (180) and (181) can be rewritten using the structure equation, Eq. (182), as

$$
\begin{array}{lll}
\widehat{D}_{[A} \widehat{F}_{B C]}=0 & \Leftrightarrow & \widehat{V}_{[A} \widehat{\mathfrak{F}}_{B C]}^{D}-\widehat{\mathfrak{F}}_{[B C}^{E} \star \widehat{\mathfrak{F}}_{A] E} D \\
\widehat{D}^{A} \widehat{F}_{A B}=0 & \Leftrightarrow & \eta^{A B}\left(\widehat{V}_{A} \widehat{\mathfrak{F}}_{B C}{ }^{D}-\widehat{\mathfrak{F}}_{B C}{ }^{E} \star \widehat{\mathfrak{F}}_{A E}{ }^{D}\right)=0 .
\end{array}
$$

We take a commutative limit $|\theta| \rightarrow 0$ (in the same sense as $\hbar \rightarrow 0$ in quantum mechanics), and we keep only the leading term in Eq. (163) for the generalized vector fields $\widehat{V}_{A}$. In this limit, we will recover the Einstein gravity for the emergent metric in Eq. (169) where Eqs. (183) and (184) reduce to the first Bianchi identity for Riemann tensors and the Einstein equations, respectively, as we checked in the previous section. The Einstein gravity is relevant only in this limit. If $|\theta|$ is finite (in the same sense as $\hbar \rightarrow 1$ in quantum mechanics), we have to rely on Eqs. (183) and (184) instead: What is going on here? In order to answer the question, it is necessary to solve Eqs. (183) and (184) first. Of course, it will be, in general, very difficult to solve the equations. Instead, one may introduce linear algebraic conditions of $D$-dimensional field strengths $\widehat{F}_{A B}$ as a higher-dimensional analogue of 4-dimensional self-duality equations such that the Yang-Mills equations in the action in Eq. (161) follow automatically. These are of the type [70]

$$
\frac{1}{2} T_{A B C D} \widehat{F}_{C D}=\chi \widehat{F}_{A B}
$$


with a constant 4-form tensor $T_{A B C D}$. The relation in Eq. (185) clearly implies via the Bianchi identity in Eq. (180) that the equations of motion, Eq. (181), are satisfied provided $\chi$ is nonzero. For $D>4$, the 4-form tensor $T_{A B C D}$ cannot be invariant under $S O(D)$ transformations and Eq. (185) breaks the rotational symmetry to a subgroup $H \subset S O(D)$. Thus, the resulting first-order equations can be classified by the unbroken symmetry $H$ under which $T_{A B C D}$ remains invariant [70]. It was also shown [71] that the first-order linear equations above are closely related to supersymmetric states, i.e., BPS states in higher-dimensional Yang-Mills theories.

Note that

$$
\widehat{V}_{-i\left[D_{A}, D_{B}\right]_{\star}}=\widehat{V}_{\widehat{F}_{A B}}=\left[\widehat{V}_{A}, \widehat{V}_{B}\right]_{\star}
$$

Using the homomorphism in Eq. (186), one can translate the generalized self-duality equation, Eq. (185), into the structure equation between vector fields,

$$
\frac{1}{2} T_{A B C D} \widehat{F}_{C D}=\chi \widehat{F}_{A B} \quad \Leftrightarrow \quad \frac{1}{2} T_{A B C D}\left[\widehat{V}_{C}, \widehat{V}_{D}\right]_{\star}=\chi\left[\widehat{V}_{A}, \widehat{V}_{B}\right]_{\star} .
$$

Therefore, a $D$-dimensional noncommutative gauge field configuration satisfying the first-order system defined by the left-hand side of Eq. (187) is isomorphic to a $D$-dimensional emergent "quantum" geometry defined by the right-hand side of Eq. (187) whose metric in the commutative limit is given by Eq. (169). For example, in four dimensions where $T_{A B C D}=\varepsilon_{A B C D}$ and $\chi= \pm 1$, Eq. (187) goes to Eq. (88) describing gravitational instantons in the commutative limit. Hence, it would not be absurd for someone to claim that self-dual noncommutative electromagnetism in four dimensions is equivalent to self-dual quantum gravity [4, 38]. Indeed, it was argued in Ref. [6] that the emergent geometry arising from the self-dual system in Eq. (187) is closely related to the bubbling geometry in the AdS space found in Ref. [33].

\section{Background-independent Quantum Gravity}

According to Einstein, gravity is the dynamics of spacetime geometry. Therefore, as emphasized by Elvang and Polchinski [72], the emergence of gravity necessarily requires the emergence of spacetime itself. That is, spacetime is not given a priori, but should be derived from fundamental ingredients in quantum gravity theory, say, "spacetime atoms". However, for consistency, the entire spacetime including a flat spacetime must be emergent. In other words, the emergent gravity should necessarily be "background independent," where any spacetime structure is not $a$ 
priori assumed, but is defined by the theory. Furthermore, if spacetime is emergent, then all fields supported on this spacetime must be emergent, too. The question is how everything, including spacetime, gauge fields and matter fields, could be emergent collectively. We know emergent phenomena in condensed matters arise due to a very coherent condensation in vacuum. Thus, in order to realize all these emergent phenomena, the emergent spacetime needs to be derived from an extremely coherent vacuum, which is the lesson we learned from condensed matter. This turns out to be the case if a flat spacetime is emergent from a noncommutative algebra such as quantum harmonic oscillators.

We will carefully recapitulate the emergent gravity derived from the action in Eq. (151) to throw the universe into a fresh perspective and to elucidate how the emergent gravity based on the noncommutative geometry achieves background independence. Of course, real physics is necessarily background dependent because a physical phenomenon occurs in a particular background with specific initial conditions. Background independence here means that, although physical events occur in a particular (spacetime and material) background, an underlying theory itself describing such a physical event should presuppose neither any kind of spacetime nor material backgrounds. The background in itself should also arise from a solution of the underlying theory.

The $U(N)$ gauge theory in Eq. (151) is defined by a collection of $N \times N$ matrices $\left(A_{\mu}, \Phi^{a}\right)(z)$ on a $d$-dimensional flat spacetime $\mathbb{R}_{C}^{d}$. Note that the $d$-dimensional flat spacetime $\mathbb{R}_{C}^{d}$ already exists from the beginning independently of $U(N)$ gauge fields and that the theory says nothing about its origin. It just serves as a playground for the players $\left(A_{\mu}, \Phi^{a}\right)$.

We showed that the $d$-dimensional matrix theory in Eq. (151) in the $N \rightarrow \infty$ limit could be mapped to the $D=d+2 n$-dimensional noncommutative $U(1)$ gauge theory. The resulting higher-dimensional $U(1)$ gauge theory has been transformed to a theory of higher-dimensional gravity describing a dynamical spacetime geometry according to the isomorphism between the noncommutative $\star$-algebra $\mathcal{A}_{\theta}$ and the algebra $\operatorname{Der}\left(\mathcal{A}_{\theta}\right)$ of vector fields. Look at the metric in Eq. (169). Definitely, the extra $2 n$-dimensional spacetime is emergent and takes part in the spacetime geometry. It was not a preexisting spacetime background in the action in Eq. (151). Instead the theory says that it originated from the vacuum in Eq. (152). One can easily check this fact by turning off all fluctuations in the metric in Eq. (169). The $D$-dimensional flat spacetime comes from the vacuum configuration in Eq. (152) whose vector field is given by $V_{A}^{(\mathrm{vac})}=\left(\partial_{\mu}, \partial_{a}\right)$ according to Eq. (163). Furthermore, the vacuum is a solution of the theory in Eq. (151). Therefore, the underlying theory in Eq. (151) by itself entirely describes the emergence of the $2 n$-dimensional 
space and its dynamical fluctuations.

Also, note that the original $d$-dimensional spacetime is now dynamical, not a playground any more, although the original flat spacetime part $\mathbb{R}_{C}^{d}$ was assumed a priori at the outset. One can see that the existence of nontrivial gauge field fluctuations $A_{\mu}(z)$ causes the curving of $\mathbb{R}_{C}^{d}$. Therefore, the large- $N$ gauge theory in Eq. (151) almost provides a background-independent description of spacetime geometry, except the original background $\mathbb{R}_{C}^{d}$.

Now, a question is how to achieve a complete background independence about the emergent geometry. The answer is simple. We may completely remove the spacetime $\mathbb{R}_{C}^{d}$ from the action in Eq. (151) and start with a theory without spacetime from the beginning. How to do this operation is well-known in matrix models. This change of dimensionality appears in matrix theory as the 'matrix T-duality' (see Sec. VI.A in Ref. [20]) defined by

$$
i D_{\mu}=i \partial_{\mu}+A_{\mu} \rightleftarrows \Phi^{a}
$$

Applying the matrix T-duality in Eq. (188) to the action in Eq. (151), on one hand, one can arrive at the 0-dimensional IKKT matrix model [18] in the case of the Euclidean signature

$$
S_{I K K T}=-\frac{2 \pi}{g_{s} \kappa^{2}} \operatorname{Tr}\left(\frac{1}{4}\left[X^{M}, X^{N}\right]\left[X_{M}, X_{N}\right]\right)
$$

where $X^{M}=\kappa \Phi^{M}$, or the 1-dimensional BFSS matrix model [17] in the case of the Lorentzian signature

$$
S_{B F S S}=-\frac{1}{G_{s}} \int d t \operatorname{Tr}\left(\frac{1}{2} D_{0} \Phi^{a} D^{0} \Phi^{a}-\frac{1}{4}\left[\Phi^{a}, \Phi^{b}\right]^{2}\right) .
$$

On the other hand, one can also go up to $D$-dimensional pure $U(N)$ Yang-Mills theory given by

$$
S_{C}=-\frac{1}{4 g_{Y M}^{2}} \int d^{D} X \operatorname{Tr} F_{M N} F^{M N} .
$$

Note that the $B$-field has completely disappeared; i.e., the spacetime is commutative. In fact, the T-duality between the theories defined by Eqs. (161) and (191) is an analogue of the Morita equivalence on a noncommutative torus stating that the noncommutative $U(1)$ gauge theory with rational $\theta=M / N$ is equivalent to an ordinary $U(N)$ gauge theory [16].

Let us focus on the IKKT matrix model in Eq. (189) because it is completely background independent because it is 0-dimensional. In order to define the action in Eq. (189), it is not necessary to assume the prior existence of any spacetime structure. There are only a bunch of 
$N \times N$ Hermitian matrices $X^{M}(M=1, \cdots, D)$ that are subject to a couple of algebraic relations given by

$$
\begin{aligned}
& {\left[X^{M},\left[X^{N}, X^{P}\right]\right]+\left[X^{N},\left[X^{P}, X^{M}\right]\right]+\left[X^{P},\left[X^{M}, X^{N}\right]\right]=0,} \\
& {\left[X_{M},\left[X^{M}, X^{N}\right]\right]=0 .}
\end{aligned}
$$

Suppose that a vacuum of the theory in Eq. (189) in the $N \rightarrow \infty$ limit is given by

$$
\left[X^{M}, X^{N}\right]=i \theta^{M N} \mathbf{1}_{N \times N}=\left(\begin{array}{cc}
0 & 0 \\
0 & i \theta^{a b}
\end{array}\right) \mathbf{1}_{N \times N},
$$

where $\theta^{a b}$ is a constant matrix of rank $2 n$. In exactly the same way as the case for Eq. (151), one can map the $N \times N$ matrices $X^{M}=\left(X^{\mu}, X^{a}\right)$ into noncommutative fields according to the correspondence in Eq. (22):

$$
\left(X^{\mu}, X^{a}\right)_{N \times N} \quad \mapsto \quad \kappa\left(\widehat{\Phi}^{\mu}(y), \frac{i}{\kappa} \theta^{a b} \widehat{D}_{b}(y)\right) \in \mathcal{A}_{\theta},
$$

where $\widehat{D}_{a}(y) \in \mathcal{A}_{\theta}$ is given by Eq. (131). It is then straightforward to get a $2 n$-dimensional noncommutative $U(1)$ gauge theory from the matrix action in Eq. (189):

$$
\widehat{S}=\frac{1}{g_{Y M}^{2}} \int d^{2 n} y \sqrt{\widehat{g}}\left(\frac{1}{4} \widehat{g}^{a c} \widehat{g}^{b d}\left(\widehat{F}_{a b}-B_{a b}\right) \star\left(\widehat{F}_{c d}-B_{c d}\right)+\frac{1}{2} \widehat{g}^{a b} \widehat{D}_{a} \widehat{\Phi}^{\mu} \star \widehat{D}_{b} \widehat{\Phi}^{\mu}-\frac{1}{4}\left[\widehat{\Phi}^{\mu}, \widehat{\Phi}^{\nu}\right]_{\star}^{2}\right),
$$

where $g_{Y M}^{2}$ and the metric $\widehat{g}^{a b}$ are defined by Eqs. (38), (39) and (40). If $\theta^{M N}$ in Eq. (194) is a constant matrix of rank $D=d+2 n$ instead, we will get a $D$-dimensional noncommutative $U(1)$ gauge theory whose action is basically the same as Eq. (161) except that it comes with the Euclidean signature and a constant $B$-field of rank $D$.

In summary, we have scanned both $U(N)$ Yang-Mills theories and noncommutative $U(1)$ gauge theories in various dimensions and different $B$-field backgrounds by applying the matrix T-duality in Eq. (188) and the correspondence in Eq. (22). From the derivation of Eq. (161), one may notice that the rank of the $B$-field is equal to the dimension of the emergent space, which is also equal to the number of adjoint scalar fields $\Phi^{a} \in U(N)$. Therefore, the matrix theory in Eq. (151) can be defined in different dimensions by changing the rank of the $B$-field if the dimension $D$ is fixed, e.g., $D=10$. On the other hand, we can change the dimensionality of the theory in Eq. (196) by changing the rank of $\theta$ in Eq. (194). In this way, we can connect every $U(N)$ Yang-Mills theory and noncommutative $U(1)$ gauge theory in various dimensions by changing the $B$-field background and applying the matrix T-duality (188) and the correspondence in Eq. (22). It is really remarkable! 
However, there is also a caveat. One can change the dimensionality of the matrix model by any integer number by using the matrix T-duality in Eq. (188) while the rank of the $B$-field can be changed only by an even number because it is supposed to be symplectic. Hence, it is not obvious what kind of background can explain a noncommutative field theory with an odd number of adjoint Higgs fields. A plausible guess is that either the vacuum is described by a noncommutative space induced by a Poisson structure, e.g., of Lie algebra type, i.e., $\left[X^{a}, X^{b}\right]=i f{ }_{c}^{a b} X^{c}$, or there is a 3-form $C_{\mu \nu \rho}$ that reduces to the 2-form $B$ in Eq. (38) by a circle compactification, so may be of M-theory origin. We will briefly discuss the Lie algebra case later, but, unfortunately, we don't know much about how to construct a corresponding noncommutative field theory with the 3-form background. We leave it as a future problem.

Some critical aspect of quantum geometry may be encountered with the following question. What is the emergent geometry derived from the noncommutative $U(1)$ gauge theory in Eq. (196) ? One may naively apply the map in Eq. (163) to the noncommutative fields $\left(\widehat{\Phi}^{\mu}(y), \widehat{D}_{a}(y)\right) \in \mathcal{A}_{\theta}$. The fields $\widehat{D}_{a}(y)$ have no problem because they are exactly the same as Eq. (131). However, the fields $\widehat{\Phi}^{\mu}(y)$ leads to a bizarre circumstance. From the map in Eq. (163), we may define

$$
\begin{aligned}
-i\left[\widehat{\Phi}^{\mu}(y), \widehat{f}(y)\right]_{\star} & =-\theta^{a b} \frac{\partial \Phi^{\mu}(y)}{\partial y^{b}} \partial_{a} f(y)+\cdots \\
& \equiv " V^{\mu a}(y) \partial_{a} f(y)+\cdots
\end{aligned}
$$

We immediately get into trouble if we remember that the fields $\widehat{\Phi}^{\mu}(y)$ are purely fluctuations and so the 'fake' vector fields $V^{\mu}=V^{\mu a}(y) \partial_{a}$ are not invertible, in general. For example, they tend to vanish at $|y| \rightarrow \infty$. Recall that a Riemannian metric should be nondegenerate, i.e., invertible everywhere. This is not the case for $V^{\mu}$. Therefore, the fields $\widehat{\Phi}^{\mu}(y)$ are not yet full-fledged as a classical geometry although they could define a "bubbling quantum geometry". This notable difference between $\widehat{\Phi}^{\mu}(y)$ and $\widehat{D}_{a}(y)$ is due to the fact that $\widehat{D}_{a}(y)$ define fluctuations around the uniform vacuum condensation in Eq. (152) while $\widehat{\Phi}^{\mu}(y)$ define pure fluctuations around "nothing", say, without any coherent condensation in vacuum. Therefore we get a very important picture from the above analysis:

In order to describe a classical geometry from a background independent theory such as Eq. (189), it is necessary to have a nontrivial vacuum defined by a "coherent" condensation of gauge fields, e.g., the vacuum defined by Eq. (152).

Here, "coherent" means that a spacetime vacuum is defined by the Heisenberg algebra such as quantum harmonic oscillators as in Eq. (17). Its physical significance will be discussed later. 
Also, note that a symplectic structure $B_{a b} \equiv\left(\theta^{-1}\right)_{a b}$ is nowhere vanishing, which can be regarded as a background field strength of noncommutative gauge fields $A_{a}^{(0)} \equiv\left\langle\widehat{A}_{a}^{(0)}\right\rangle_{\mathrm{vac}}=-B_{a b} y^{b}$. In terms of physicist's language, this means that there is an (inhomogeneous in general) condensation of gauge fields in vacuum, i.e.,

$$
\left\langle B_{a b}(x)\right\rangle_{\mathrm{vac}}=\theta_{a b}^{-1}(x)
$$

For a constant symplectic structure for simplicity, rewriting the covariant vectors in Eq. (131) as (actually to invoke a renowned Goldstone boson $\varphi=\langle\varphi\rangle+h$ ) [73]

$$
\widehat{D}_{a}(y)=-\left\langle\widehat{A}_{a}^{(0)}\right\rangle_{\mathrm{vac}}+\widehat{A}_{a}(y)
$$

would be suggestive. This naturally suggests some sort of spontaneous symmetry breaking [5] in which $y^{a}$ are vacuum expectation values of $\widehat{D}_{a}(y)$, specifying the background in Eq. (198)

as usual, and $\widehat{A}_{b}(y)$ are fluctuating dynamical coordinates (fields). We thus arrived at another important point:

The origin of spacetime with a symplectic or a Poisson structure such as Eq. (6) or Eq. (17) comes from the coherent condensation of gauge fields in vacuum.

\section{General Noncommutative Spacetime}

So far, we have mostly considered noncommutative spaces defined by a canonical symplectic structure. Here, we will explain how it is possible to generalize emergent gravity to a general noncommutative spacetime, for example, to the case with a nonconstant symplectic structure or a generic Poisson structure. General results have been beyond our reach up to now. Thus, we will be brief about this subject. Readers may skip this part and might attack the emergent gravity for general cases after a deeper understanding about the simple cases has been realized.

The question is how to generalize the emergent gravity picture to the case of a nontrivial vacuum, e.g., Eq. (198), describing an inhomogeneous condensate of gauge fields. In this case, the Poisson structure $\Theta^{a b}(x)=\left(\frac{1}{B}\right)^{a b}(x)$ is not constant, so the corresponding noncommutative field theory is defined by a nontrivial star-product

$$
\left[Y^{a}, Y^{b}\right]_{\star^{\prime}}=i \Theta^{a b}(Y)
$$

where $Y^{a}$ denote vacuum coordinates, which are designed with the capital letters to distinguish them from $y^{a}$ for the constant vacuum in Eq. (17). The star product $[\widehat{f}, \widehat{g}]_{\star^{\prime}}$ for $\widehat{f}, \widehat{g} \in \mathcal{A}_{\Theta}$ can be 
perturbatively computed via the deformation quantization [15]. There are excellent earlier works [75] especially relevant for the analysis of the DBI action as a generalized geometry though a concrete formulation of noncommutative field theories for a general noncommutative spacetime is still out of reach.

We will mostly focus on the commutative limit so that

$$
\begin{aligned}
-i[\widehat{f}, \widehat{g}]_{\star^{\prime}} & =\Theta^{a b}(Y) \frac{\partial f(Y)}{\partial Y^{a}} \frac{\partial g(Y)}{\partial Y^{b}}+\cdots \\
& \equiv\{f, g\}_{\Theta}+\cdots
\end{aligned}
$$

for $\widehat{f}, \widehat{g} \in \mathcal{A}_{\Theta}$. Using the Poisson bracket in Eq. (201), we can similarly realize the Lie algebra homomophism $C^{\infty}(M) \rightarrow T M: f \mapsto X_{f}$ between a Hamiltonian function $f$ and the corresponding Hamiltonian vector field $X_{f}$. To be specific, for any given function $f \in C^{\infty}(M)$, we can always assign a Hamiltonian vector field $X_{f}$ defined by $X_{f}(g)=\{g, f\}_{\Theta}$ with any fixed function $g \in C^{\infty}(M)$. Then, the Lie algebra homomophism

$$
X_{\{f, g\}_{\Theta}}=\left[X_{f}, X_{g}\right]
$$

still holds as long as the Jacobi identity for the Poisson bracket $\{f, g\}_{\Theta}(x)$ holds or, equivalently, the Schouten-Nijenhuis bracket in Eq. (223) for the Poisson structure $\Theta^{a b}$ vanishes.

As we discussed in Eq. (140), there is a natural automorphism $D(\hbar)$ that acts on star-products [15]. In the commutative limit where $D(\hbar) \approx 1$, we can deduce the following relation from Eq. (140):

$$
\{f, g\}_{\Theta}=\{f, g\}_{\theta}
$$

Let us explain what Eq. (203) means. For $f=Y^{a}(y)$ and $g=Y^{b}(y)$, Eq. (203) implies that

$$
\Theta^{a b}(Y)=\theta^{c d} \frac{\partial Y^{a}}{\partial y^{c}} \frac{\partial Y^{b}}{\partial y^{d}}
$$

whose statement is, of course, equivalent to the Darboux transformation in Eq. (29). Also, notice that Eq. (203) defines diffeomorphisms between vector fields $X_{f}^{\prime}(g) \equiv\{g, f\}_{\Theta}$ and $X_{f}(g) \equiv$ $\{g, f\}_{\theta}$ such that

$$
X_{f}^{\prime a}=\frac{\partial Y^{a}}{\partial y^{b}} X_{f}^{b}
$$

Indeed, the automorphism in Eq. (140) corresponds to a global statement that the two star-products involved are cohomologically equivalent in the sense that they generate the same Hochschild cohomology [15]. 
In order to understand the origin of the nontrivial star product in Eq. (200), let us look at the background independent action in Eq. (189). As we pointed out, a particular vacuum such as the one in Eq. (198) should be defined by the theory itself as a solution of the equations of motion, Eq. (193). Of course, there are infinitely many solutions. The constant background in Eq. (194) is just one of them, so let us consider another background

$$
\left[X^{M}, X^{N}\right]=\left(\begin{array}{cc}
0 & 0 \\
0 & i(\theta-\theta \widehat{F} \theta)^{a b}
\end{array}\right) \mathbf{1}_{N \times N} .
$$

Using the property in Eq. (203), one can infer that the above background can be made equivalent to Eq. (200) by using the identification $X^{a}:=Y^{a}=y^{a}+\theta^{a b} \widehat{A}_{b}(y)$ and $\Theta^{a b}(y)=(\theta-\theta \widehat{F}(y) \theta)^{a b}$. If $\widehat{F}_{a b}(y)$ simply satisfy Eq. (185), which provides a very ample class of solutions, the background in Eq. (206) is a consistent solution of the theory in Eq. (189). For example, the vacuum in Eq. (206) in four dimensions $(n=2)$ corresponds to the noncommutative instanton background. In this case, the vacuum manifold determined by background gauge fields is a hyper-Kähler manifold.

Therefore, we may understand that the nontrivial star product in Eq. (200) results from an inhomogeneous condensation of gauge fields on the constant vacuum in Eq. (194). This observation can be applied to the identity in Eq. (36) in a very interesting way. Let us decompose the nontrivial B-field in Eq. (198) as

$$
B_{a b}(x)=(\bar{B}+\bar{F}(x))_{a b},
$$

where $\bar{B}_{a b}=\left(\theta^{-1}\right)_{a b}$ describes a constant background such as the one in Eq. (17) while $\bar{F}(x)=$ $d \bar{A}(x)$ describes an inhomogeneous condensate of gauge fields. Then, the left-hand side of Eq. (36) is of the form $g+\kappa(\bar{B}+\mathcal{F})$, where $\mathcal{F}=d \mathcal{A}$ with $\mathcal{A}(x)=\bar{A}(x)+A(x)$. It should be completely conceivable that it can be mapped to a noncommutative gauge theory of the gauge field $\mathcal{A}(x)$ in the constant $\bar{B}$-field background according to the Seiberg-Witten equivalence [16]. Let us denote the corresponding noncommutative gauge field as $\widehat{\mathcal{A}}_{a} \equiv \widehat{\bar{A}}_{a}+\widehat{A}_{a}$. The only notable point is that the gauge field $\widehat{\mathcal{A}}_{a}$ contains an inhomogeneous background part $\widehat{\bar{A}}_{a}$. This situation is, of course, analogous to an instanton (or soliton) background in gauge theory, as we remarked before.

Thus, everything will go parallel with the constant case. We will consider a general situation in the context of the action in Eq. (161), where background gauge fields are given by $\widehat{\bar{A}}_{\mu}(z, y)$ as well as $\widehat{\bar{A}}_{b}(z, y)$, which also depend on the commutative coordinates $z^{\mu}$. Let us introduce the 
following covariant coordinates:

$$
\begin{aligned}
\widehat{X}^{a}(z, y) & =y^{a}+\theta^{a b} \widehat{\mathcal{A}}_{b}(z, y)=y^{a}+\theta^{a b} \widehat{\widehat{A}}_{b}(z, y)+\theta^{a b} \widehat{A}_{b}(z, y) \\
& \equiv Y^{a}(z, y)+\theta^{a b} \widehat{A}_{b}(z, y),
\end{aligned}
$$

where we identified the vacuum coordinates $Y^{a}$ in Eq. (200) because we have to recover them after turning off the fluctuation $\widehat{A}_{a}$. Also, introduce the covariant derivatives

$$
\begin{aligned}
\widehat{D}_{\mu}(z, y) & =\partial_{\mu}-i \widehat{\mathcal{A}}_{\mu}(z, y)=\partial_{\mu}-i \widehat{\bar{A}}_{\mu}(z, y)-i \widehat{A}_{\mu}(z, y) \\
& \equiv \widehat{\bar{D}}_{\mu}(z, y)-i \widehat{A}_{\mu}(z, y)
\end{aligned}
$$

Then, the covariant derivatives in Eq. (157) can be defined in exactly the same way:

$$
\widehat{D}_{A}=\partial_{A}-i \widehat{\mathcal{A}}_{A}(z, y)=\left(\widehat{D}_{\mu},-i \bar{B}_{a b} \widehat{X}^{b}\right)(z, y)
$$

where $\partial_{A}=\left(\partial_{\mu},-i \bar{B}_{a b} y^{b}\right)$. Now, the noncommutative fields $\widehat{D}_{A}$ in Eq. (210) can be mapped to vector fields using Eq. (163).

Because the results in Section III.B can be applied to arbitrary noncommutative gauge fields in a constant $B$-field, the same formulae can be applied to the present case with the understanding that the vector fields $V_{A}$ in Eq. (163) refer to total gauge fields including the inhomogeneous background. This means that the vector fields $V_{A}=\lambda E_{A} \in \Gamma(T M)$ reduce to $\bar{V}_{A}=\bar{\lambda} \bar{E}_{A}$ after turning off the fluctuations, where $\bar{V}_{A}$ is determined by the background $\left(\partial_{\mu}-i \widehat{\bar{A}}_{\mu}(z, y),-i \bar{B}_{a b} Y^{b}(z, y)\right)$ and $\bar{\lambda}$ satisfies the relation

$$
\bar{\lambda}^{2}=\nu\left(\bar{V}_{1}, \cdots, \bar{V}_{D}\right) .
$$

Therefore, the $D$-dimensional metric is precisely given by Eq. (169) with $\mathbf{A}^{a}=\mathcal{A}_{\mu}^{a} d z^{\mu}$, and the metric for the background is given by

$$
\begin{aligned}
d s^{2} & =\eta_{A B} \bar{E}^{A} \otimes \bar{E}^{B} \\
& =\bar{\lambda}^{2} \eta_{A B} \bar{V}^{A} \otimes \bar{V}^{B}=\bar{\lambda}^{2} \eta_{A B} \bar{V}_{M}^{A} \bar{V}_{N}^{B} d X^{M} \otimes d X^{N}
\end{aligned}
$$

Here, we have implicitly assumed that the background $\bar{V}_{A}$ satisfies Eqs. (180) and (181). In four dimensions, for instance, we know that the metric in Eq. (212) describes Ricci-flat Kähler manifolds if $\bar{V}_{A}$ satisfies the self-duality equation, Eq. (88).

Now, let us look at the picture of the right-hand side of Eq. (36). After applying the Darboux transform, Eq. (29), only for the symplectic structure, Eq. (207), and leaving the fluctuations 
intact, the right-hand side becomes of the form $G_{a b}(y)+\kappa\left(\bar{B}_{a b}+\mathfrak{F}_{a b}(y)\right)$, where

$$
\mathfrak{F}_{a b}(y)=\frac{\partial x^{\alpha}}{\partial y^{a}} \frac{\partial x^{\beta}}{\partial y^{b}} F_{\alpha \beta}(x) \equiv \partial_{a} \mathfrak{A}_{b}(y)-\partial_{b} \mathfrak{A}_{a}(y),
$$

and the metric $G_{a b}(y)$ is given by Eq. (33). Note that in this picture, the gauge fields $\mathfrak{A}_{a}(y)$ are regarded as fluctuations propagating in the background $G_{a b}(y)$ and $\bar{B}_{a b}$. Therefore, it would be reasonable to interpret the right-hand side of Eq. (36) as a noncommutative gauge theory of the gauge field $\mathfrak{A}_{a}(y)$ defined by the canonical noncommutative space in Eq. (17), but in a curved space described by the metric $G_{a b}(y)$.

Although the formulation of noncommutative field theory in a generic curved spacetime is still a challenging problem, there is no obstacle to formulating emergent gravity if one is confined to the commutative limit. Because the inhomogeneous condensate of gauge fields in the vacuum (207) now appears as an explicit background metric, the metric in Eq. (169) in this picture will be replaced by

$$
\begin{aligned}
d s^{2} & =h_{A B} E^{A} \otimes E^{B} \\
& =\Lambda^{2} h_{A B} V^{A} \otimes V^{B}=\Lambda^{2} h_{A B} V_{M}^{A} V_{N}^{B} d X^{M} \otimes d X^{N},
\end{aligned}
$$

where $h_{A B}$ is the metric in the space spanned by noncoordinate bases $V_{A}=\Lambda E_{A}$ [76]. Because the metric in Eq. (214) has the Riemannian volume form $\nu_{g}=\sqrt{-h} E^{1} \wedge \cdots \wedge E^{D}$ instead of Eq. (172), the volume form $\nu=\Lambda^{(2-D)} \nu_{g}$ in Eq. (170) will be given by

$$
\nu=\sqrt{-h} \Lambda^{2} V^{1} \wedge \cdots \wedge V^{D}
$$

Thus, the function $\Lambda$ in Eq. (214) will be determined by the condition

$$
\sqrt{-h} \Lambda^{2}=\nu\left(V_{1}, \cdots, V_{D}\right)
$$

Because the anholonomic basis $V^{A}$ in Eq. (214) will become flat when fluctuations are turned off, i.e., $\mathfrak{F}_{a b}=0$, the background metric in this picture is simply given by

$$
d s^{2}=\bar{\Lambda}^{2} h_{M N} d X^{M} \otimes d X^{N}
$$

where $\bar{\Lambda}^{2}=1 / \sqrt{-h}$.

As usual, the torsion-free condition, Eq. (102), for the metric in Eq. (214) will be imposed to get the relation in Eq. (103) in which $\omega_{A B C}=h_{B D} \omega_{A}{ }^{D}$ and $f_{A B C}=h_{C D} f_{A B}{ }^{D}$, where the indices $A, B, \cdots$ are raised and lowered using the metric $h_{A B}$. Because $h_{A B}$ is not a flat metric, 
$\omega_{A}{ }^{B}$ in Eq. (100) or Eq. (101) will actually be the Levi-Civita connection in noncoordinate bases rather than a spin connection, but we will keep the notation for convenience. Also, the condition that the metric in Eq. (214) be covariantly constant, i.e., $\nabla_{C}\left(h_{A B} E^{A} \otimes E^{B}\right)=0$, leads to the relation [76]

$$
\omega_{A B C}=\frac{1}{2}\left(E_{A} h_{B C}-E_{B} h_{C A}+E_{C} h_{A B}\right)+\frac{1}{2}\left(f_{A B C}-f_{B C A}+f_{C A B}\right) .
$$

The curvature tensors have exactly the same form as Eq. (105). All the calculation in Section II.C can be repeated in the same way even for this case although the details will be much more complicated and have not been performed so far. By comparing the two metrics, Eqs. (212) and (217), we finally get the following relations [7]:

$$
h_{M N}=\eta_{A B} \bar{V}_{M}^{A} \bar{V}_{N}^{B}, \quad \bar{\Lambda}^{2}=\bar{\lambda}^{2}=\frac{1}{\sqrt{-h}},
$$

which is, of course, consistent with our earlier observation.

One may wonder whether the emergent gravity for symplectic structures can be smoothly taken over to the case where a symplectic structure is not available. It was shown in Ref. [42] that emergent gravity can nicely be generalized to a Poisson manifold $(M, \pi)$. A Poisson manifold $M$ is a differentiable manifold $M$ equipped with a bivector field (not necessarily nondegenerate) $\pi=\pi^{\mu \nu} \partial_{\mu} \wedge \partial_{\nu} \in \Gamma\left(\Lambda^{2} T M\right)$ which defines an $\mathbb{R}$-bilinear antisymmetric operation $\{\cdot, \cdot\}_{\pi}$ : $C^{\infty}(M) \times C^{\infty}(M) \rightarrow C^{\infty}(M)$ by

$$
(f, g) \mapsto\{f, g\}_{\pi}=\langle\pi, d f \otimes d g\rangle=\pi^{\mu \nu}(x) \partial_{\mu} f(x) \partial_{\nu} g(x) .
$$

The bracket, called the Poisson bracket, satisfies

$$
\begin{aligned}
& \text { 1) Leibniz rule : }\{f, g h\}_{\pi}=g\{f, h\}_{\pi}+\{f, g\}_{\pi} h \text {, } \\
& \text { 2) Jacobi identity : }\left\{f,\{g, h\}_{\pi}\right\}_{\pi}+\left\{g,\{h, f\}_{\pi}\right\}_{\pi}+\left\{h,\{f, g\}_{\pi}\right\}_{\pi}=0 \text {, }
\end{aligned}
$$

$\forall f, g, h \in C^{\infty}(M)$. Poisson manifolds appear as a natural generalization of symplectic manifolds where the Poisson structure reduces to a symplectic structure if $\pi$ is nongenerate [8].

One can show that the Jacobi identity in Eq. (222) for the bracket $\{\cdot, \cdot\}_{\pi}$ is equivalent to the condition that the Schouten-Nijenhuis bracket [77] for the Poisson tensor $\pi$ vanishes, i.e.,

$$
[\pi, \pi]_{S N} \equiv\left(\pi^{\lambda \mu} \frac{\partial \pi^{\nu \rho}}{\partial x^{\lambda}}+\pi^{\lambda \nu} \frac{\partial \pi^{\rho \mu}}{\partial x^{\lambda}}+\pi^{\lambda \rho} \frac{\partial \pi^{\mu \nu}}{\partial x^{\lambda}}\right) \frac{\partial}{\partial x^{\mu}} \wedge \frac{\partial}{\partial x^{\nu}} \wedge \frac{\partial}{\partial x^{\rho}}=0 .
$$

Like the Darboux theorem in symplectic geometry, the Poisson geometry also enjoys a similar property known as the splitting theorem proven by Weinstein [78]. The splitting theorem states 
that a $d$-dimensional Poisson manifold is locally equivalent to the product of $\mathbb{R}^{2 n}$ equipped with the canonical symplectic structure with $\mathbb{R}^{d-2 n}$ equipped with a Poisson structure of rank zero at the origin. That is, the Poisson manifold $(M, \pi)$ is locally isomorphic (in a neighborhood of $x \in M$ ) to the direct product $S \times N$ of a symplectic manifold $\left(S, \sum_{i=1}^{n} d q^{i} \wedge d p_{i}\right)$ with a Poisson manifold $\left(N_{x},\{\cdot, \cdot\}_{N}\right)$ whose Poisson tensor vanishes at $x$.

A well-known example of a Poisson manifold is four-sphere where no symplectic structure is available. If $M$ is a compact symplectic manifold, the second de Rham cohomology group $H^{2}(M)$ is nontrivial, so the only $n$-sphere that admits a symplectic form is the 2 -sphere. For example, let $\mathbf{S}^{4}=\left\{(u, v, t) \in \mathbb{C} \times \mathbb{C} \times \mathbb{R}:|u|^{2}+|v|^{2}=t(2-t)\right\}$. Then, the bivector field $\pi=u v \partial_{u} \wedge \partial_{v}-u v^{*} \partial_{u} \wedge \partial_{v^{*}}-u^{*} v \partial_{u^{*}} \wedge \partial_{v}+u^{*} v^{*} \partial_{u^{*}} \wedge \partial_{v^{*}}$ is a Poisson tensor, that is, $[\pi, \pi]_{S N}=0$, and $\pi \wedge \pi=4|u|^{2}|v|^{2} \partial_{u} \wedge \partial_{v} \wedge \partial_{u^{*}} \wedge \partial_{v^{*}}$. Therefore, the Poisson tensor $\pi$ vanishes on a subspace of either $u=0$ or $v=0$, so the Poisson structure becomes degenerate there. In this case, we have to rely on a Poisson structure to formulate an emergent gravity [42].

The Poisson tensor $\pi$ of a Poisson manifold $M$ induces a bundle map $\pi^{\sharp}: T^{*} M \rightarrow T M$ by

$$
A \mapsto \pi^{\sharp}(A)=\pi^{\mu \nu}(x) A_{\mu}(x) \frac{\partial}{\partial x^{\nu}}
$$

for $A=A_{\mu}(x) d x^{\mu} \in T_{x}^{*} M$, which is called the anchor map of $\pi$ [77]. See also Section VI. The rank of the Poisson structure at a point $x \in M$ is defined as the rank of the anchor map at that point. If the rank equals the dimension of the manifold at each point, the Poisson structure reduces to a symplectic structure, which is also called nondegenerate. The nondegenerate Poisson structure uniquely determines the symplectic structure defined by a 2-form $\omega=\frac{1}{2} \omega_{\mu \nu}(x) d x^{\mu} \wedge d x^{\nu}=\pi^{-1}$, and the condition in Eq. (223) is equivalent to the statement that the 2 -form $\omega$ is closed, $d \omega=0$. In this case, the anchor map $\pi^{\sharp}: T^{*} M \rightarrow T M$ becomes a bundle isomorphism, as we discussed in Section I.

To define a Hamiltonian vector field $\pi^{\sharp}(d f)$ of a smooth function $f \in C^{\infty}(M)$, what one really needs is a Poisson structure that reduces to a symplectic structure for the nondegenerate case. A Hamiltonian vector field $X_{f}=-\pi^{\sharp}(d f)$ for a smooth function $f \in C^{\infty}(M)$ is defined by the anchor map in Eq. (224) as follows:

$$
X_{f}(g)=-\langle\pi, d f \otimes d g\rangle=\{g, f\}_{\pi}=\pi^{\mu \nu}(x) \frac{\partial f}{\partial x^{\nu}} \frac{\partial g}{\partial x^{\mu}} .
$$

Given a smooth Poisson manifold $(M, \pi)$, the map $f \mapsto X_{f}=-\pi^{\sharp}(d f)$ is a homomorphism [77] from the Lie algebra $C^{\infty}(M)$ of smooth functions under the Poisson bracket to the Lie algebra of 
smooth vector fields under the Lie bracket. In other words, the Lie algebra homomorphism in Eq. (10) is still true even for any Poisson manifold.

As we just noticed, it is enough to have a Poisson structure to achieve the map $C^{\infty}(M) \rightarrow$ $\Gamma(T M): f \mapsto X_{f}=-\pi^{\sharp}(d f)$ such as Eq. (9). As we discussed earlier, any Poisson manifold can be quantized via deformation quantization [15]:

$$
\left\{x^{\mu}, x^{\nu}\right\}_{\pi}=\pi^{\mu \nu}(x) \rightarrow\left[\widehat{x}^{\mu}, \widehat{x}^{\nu}\right]_{\widetilde{\star}}=i \kappa \widehat{\pi}^{\mu \nu}(\widehat{x})
$$

where we introduced a deformation parameter $\kappa$ of (length) $)^{2}$ and $\widehat{\pi}^{a b}(\widehat{x}) \in \mathcal{A}_{\pi}$ are assumed to be dimensionless operators. Therefore, the anchor map in Eq. (225) can be lifted to a noncommutative manifold as in Eq. (144),

$$
\widehat{V}_{a}[\widehat{f}](x) \equiv-i\left[\widehat{D}_{a}(x), \widehat{f}(x)\right]_{\widetilde{\star}},
$$

for any noncommutative field $\widehat{D}_{a}(x) \in \mathcal{A}_{\pi}$ (dropping the hat in the coordinates $\widehat{x}^{\mu} \in \mathcal{A}_{\pi}$ for simple notation). Then, everything will go exactly parallel with the symplectic case if we define emergent quantum gravity from a gauge theory defined on the noncommutative space in Eq. (226) with the generalized vector fields in Eq. (227). It was studied in Ref. [42] how a fuzzy Poisson manifold can be derived from a mass deformed matrix model, from which the picture of emergent gravity was checked.

\section{EMERGENT MATTER}

We have stressed that quantum gravity should be background independent where no kind of spacetime structure is assumed. Only morphisms between objects need to be postulate. An underlying theory, for example, only has matrices (as objects) that are subject to some algebraic relations such as the Jacobi identity and the equations of motion (as morphisms). However, we can derive a spacetime geometry from these algebraic relations between objects by mapping the matrix algebra to a Poisson or noncommutative $\star$-algebra $\mathcal{A}_{\theta}$ and then deriving the algebra $\operatorname{Der}\left(\mathcal{A}_{\theta}\right)$ of $\mathcal{A}_{\theta}$. We observed that such an operator algebra, e.g., $\star$-algebra, can be defined by using noncommutative gauge fields and that a smooth geometry emerges from them in a macroscopic world. Depending on the choice of an algebraic relation, we get a different geometry. In this scheme, the geometry is a derived concept defined by the algebra [14]. In a deep noncommutative space, a smooth geometry is doomed; instead, an algebra between objects becomes more fundamental. Ergo, the motto of 
emergent gravity is that an algebra defines a geometry. One has to specify an underlying algebra to talk about a corresponding geometry.

As a recitation, the emergence of gravity necessarily requires the emergence of spacetime itself. If spacetime is emergent, then all fields supported on this spacetime must be emergent too. Somehow, matter fields and other non-Abelian gauge fields for weak and strong forces must be emergent together with spacetime. How is this possible? How are matter fields describing quarks and leptons to be defined in the context of emergent geometry?

We may start with a naive reasoning. First, note that translations in noncommutative directions are an inner automorphism of the noncommutative $\star$-algebra $\mathcal{A}_{\theta}$ generated by the coordinates in Eq. (17):

$$
e^{-i k^{a} B_{a b} y^{b}} \star \widehat{f}(y) \star e^{i k^{a} B_{a b} y^{b}}=\widehat{f}(y+k)
$$

for any $\widehat{f}(y) \in \mathcal{A}_{\theta}$. The inner automorphism in Eq. (228) is nontrivial only in the case of a noncommutative algebra [9]; that is, commutative algebras do not possess any inner automorphism, so all "points" in noncommutative space are indistinguishable, i.e., unitarily equivalent while all points in commutative space are distinguishable, i.e., unitarily inequivalent. As a result, one loses the meaning of "point" in noncommutative space. Hence, the concept of "particle" becomes ambiguous, too. Thus, before matter fields, first we may address the question: What is a particle in noncommutative spacetime?

When a space becomes noncommutative, there is a Hilbert space $\mathcal{H}$ associated with the space such as Eq. (18), so a point or a particle may be replaced by a state in $\mathcal{H}$. Then, the most natural concept of a particle in noncommutative space may be a localized state in $\mathcal{H}$. However, because the Hilbert space $\mathcal{H}$ is a complex vector space as usual, such a localized state will tend to be dissipative due to a linear superposition between nearby states. Therefore, the most natural and pertinent concept of a particle in noncommutative space may be a stable localized state in $\mathcal{H}$. This means [7] that a particle may be realized as a topological object in the noncommutative $\star$-algebra $\mathcal{A}_{\theta}$

As illustrated by quantum mechanics, noncommutative algebras admit a much greater variety of algebraic and topological structures compared to commutative ones. Likewise, when spacetime at a fundamental level is replaced by a noncommutative algebra, algebraic and topological structures in the noncommutative spacetime actually become extremely rich and coherent [14], which would, we guess, be responsible for emergent properties such as diffeomorphisms, gauge symmetries and 
matter fields.

This line of thought is our naive reasoning about how to realize a particle or matter field in noncommutative spacetime. We think this idea should direct us to a reasonable track, but an involved math is often abstruse. Therefore, we will try to get more insights from physics.

\section{A. Feynman's View on Electrodynamics}

In a very charming paper [46], Dyson explains the Feynman's view about the electrodynamics of a charged particle. Feynman starts with an assumption that a particle exists with position $x^{i}$ and velocity $\dot{x}_{i}$ satisfying commutation relations

$$
\left[x^{i}, x^{k}\right]=0, \quad m\left[x^{i}, \dot{x}_{k}\right]=i \hbar \delta_{k}^{i}
$$

Then, he asks a question: What is the most general form of forces appearing in Newton's equation $m \ddot{x}_{i}=F_{i}(x, \dot{x}, t)$ consistent with the commutation relation in Eq. 229)? Remarkably, he ends up with the electromagnetic force

$$
m \frac{d \mathbf{v}}{d t}=e(\mathbf{E}+\mathbf{v} \times \mathbf{B}) .
$$

In a sense, Feynman's result is a no-go theorem for the consistent interaction of particles in quantum mechanics. It turns out that the conditions in Eq. (229) are restrictive enough that only the electromagnetic force in Eq. (230) is compatible with them.

We here reproduce his argument with a puny refinement. We will start with the Feynman's assumption, together with the Hamilton's equation

$$
\frac{d f}{d t}=\frac{i}{\hbar}[H, f]+\frac{\partial f}{\partial t}
$$

where $f=f(x, p, t), H=H(x, p, t) \in \mathcal{A}_{\hbar}$ and $\dot{x}_{i} \equiv \dot{x}_{i}(x, p)$. However, we will not assume Newton's equation $m \ddot{x}_{i}=F_{i}(x, \dot{x}, t)$. To be precise, we replaced Newton's equation by Eq. (231), i.e.,

$$
m \frac{d \dot{x}_{i}}{d t}=\frac{i m}{\hbar}\left[H, \dot{x}_{i}\right] \equiv F_{i}(x, p, t) .
$$

First, consider the following commutator:

$$
\begin{aligned}
{\left[H,\left[x^{i}, \dot{x}_{k}\right]\right] } & =\left[x^{i},\left[H, \dot{x}_{k}\right]\right]-\left[\dot{x}_{k},\left[H, x^{i}\right]\right] \\
& =-i \hbar\left(\frac{1}{m}\left[x^{i}, F_{k}\right]+\left[\dot{x}_{i}, \dot{x}_{k}\right]\right)=0 .
\end{aligned}
$$


The Jacobi identity $\left[x^{l},\left[\dot{x}_{i}, \dot{x}_{k}\right]\right]+\left[\dot{x}_{i},\left[\dot{x}_{k}, x^{l}\right]\right]+\left[\dot{x}_{k},\left[x^{l}, \dot{x}_{i}\right]\right]=\left[x^{l},\left[\dot{x}_{i}, \dot{x}_{k}\right]\right]=0$ with Eq. (233) implies

$$
\left[x^{l},\left[x^{i}, F_{k}\right]\right]=0 .
$$

Equation (233) also implies $\left[x^{i}, F_{k}\right]+\left[x^{k}, F_{i}\right]=0$, so we may write

$$
\left[x^{i}, F_{k}\right]=-\frac{i \hbar}{m} \varepsilon^{i k l} B_{l} .
$$

Equation (235) is the definition of the field $B_{l}=B_{l}(x, p, t) \in \mathcal{A}_{\hbar}$, but Eq. (234) says

$$
\left[x^{l}, B_{m}\right]=0
$$

which means that $B_{m}$ is a function of $x$ and $t$ only, i.e., $B_{m}=B_{m}(x, t)$. Then, we can solve Eq. (235) with

$$
F_{i}(x, p, t)=E_{i}(x, t)+\varepsilon^{i k l}\left\langle\dot{x}_{k} B_{l}(x, t)\right\rangle
$$

where $E_{i}(x, t) \in \mathcal{A}_{\hbar}$ is an arbitrary function that also depends on $x$ and $t$ only and the symbol $\langle\cdots\rangle$ denotes the Weyl-ordering, i.e., the complete symmetrization of operator products.

Combining Eqs. (233) and (235) leads to

$$
B_{l}=-\frac{i m^{2}}{2 \hbar} \varepsilon^{l i k}\left[\dot{x}_{i}, \dot{x}_{k}\right]
$$

Another Jacobi identity $\varepsilon^{i j k}\left[\dot{x}_{i},\left[\dot{x}_{j}, \dot{x}_{k}\right]\right]=0$ then implies

$$
\left[\dot{x}_{i}, B_{i}\right]=-\frac{i \hbar}{m}\left\langle\frac{\partial B_{i}}{\partial x^{i}}\right\rangle=0
$$

Taking the total derivative of Eq. (238) with respect to time gives

$$
\begin{aligned}
\left\langle\dot{x}_{i} \frac{\partial B_{l}}{\partial x^{i}}\right\rangle+\frac{\partial B_{l}}{\partial t} & =\frac{m^{2}}{2 \hbar^{2}} \varepsilon^{l i k}\left[H,\left[\dot{x}_{i}, \dot{x}_{k}\right]\right] \\
& =\frac{i m}{\hbar} \varepsilon^{l i k}\left[\dot{x}_{k}, F_{i}\right] \\
& =\frac{i m}{\hbar}\left(-\varepsilon^{l i k}\left[\dot{x}_{i}, E_{k}\right]-\left[\dot{x}_{i}, \dot{x}_{l}\right] B_{i}-\dot{x}_{l}\left[\dot{x}_{i}, B_{i}\right]+\left\langle\dot{x}_{i}\left[\dot{x}_{i}, B_{l}\right]\right\rangle\right) \\
& =-\varepsilon^{l i k}\left\langle\frac{\partial E_{k}}{\partial x^{i}}\right\rangle+\frac{1}{m} \varepsilon^{l i k} B_{i} B_{k}+\left\langle\dot{x}_{i} \frac{\partial B_{l}}{\partial x^{i}}\right\rangle \\
& =-\varepsilon^{l i k}\left\langle\frac{\partial E_{k}}{\partial x^{i}}\right\rangle+\left\langle\dot{x}_{i} \frac{\partial B_{l}}{\partial x^{i}}\right\rangle .
\end{aligned}
$$

From Eq. (240), we finally get

$$
\frac{\partial B_{l}}{\partial t}+\varepsilon^{l i k}\left\langle\frac{\partial E_{k}}{\partial x^{i}}\right\rangle=0 .
$$


We arrived at the force in Eq. (237) (by using the definition in Eq. (232)), where the fields $E_{i}(x, t)$ and $B_{i}(x, t)$ should satisfy Eqs. (239) and (241). We immediately recognize that they are electromagnetic fields. Therefore, we get a remarkable result [46] that the Lorentz force in Eq. (230) is the only consistent interaction with a quantum particle satisfying the commutation relations in Eq. (229). Remember that we have only assumed the commutation relation (229) and have only used the Hamilton's equation in Eq. (231) and the Jacobi identity to find a consistent interaction of quantum particles. However, we could get only the electromagnetic force in Eq. (230). What a surprise (at least to us)!

Feynman's observation raises a curious question. We know that, beside the electromagnetic force, other interactions, weak and strong forces, exist in Nature. Thus, the question is how to incorporate the weak and the strong forces into Feynman's scheme. Because he started only with very natural axioms, there seems to be no room to relax his postulates to include the weak and the strong forces except by introducing extra dimensions. Surprisingly, it works with extra dimensions!

Consider a particle motion defined on $\mathbb{R}^{3} \times F$ with an internal space $F$ whose coordinates are $\left\{x^{i}: i=1,2,3\right\} \in \mathbb{R}^{3}$ and $\left\{Q^{I}: I=1, \cdots, n^{2}-1\right\} \in F$. The dynamics of the particle carrying an internal charge in $F[47,48]$ is defined by a symplectic structure on $T^{*} \mathbf{R}^{3} \times F$ whose commutation relations are given by

$$
\begin{aligned}
& {\left[x^{i}, x^{k}\right]=0, \quad m\left[x^{i}, \dot{x}_{k}\right]=i \hbar \delta_{k}^{i},} \\
& {\left[Q^{I}, Q^{J}\right]=i \hbar f_{K}^{I J}{ }_{K}^{K},} \\
& {\left[x^{i}, Q^{I}\right]=0 .}
\end{aligned}
$$

Note that the internal space $F$ is a Poisson manifold $(F, \pi)$ whose Poisson structure is given by $\pi=\frac{1}{2} \pi^{I J} \partial_{I} \wedge \partial_{J}=\frac{1}{2} f^{I J}{ }_{K} Q^{K} \partial_{I} \wedge \partial_{J}$ and defines the $S U(n)$ Lie algebra in Eq. (243). That is, by Eq. (223),

$$
[\pi, \pi]_{S N}=0 \quad \Leftrightarrow \quad f^{J K}{ }_{L} f^{L I}{ }_{M}+f^{K I}{ }_{L} f_{M}^{L J}+f_{L}^{I J} f_{M}^{L K}=0 .
$$

Also, the internal coordinates $Q^{I}$ are assumed to obey Wong's equation [79]

$$
\dot{Q}^{I}+f_{J K}^{I} A_{i}^{J}(x, t) Q^{K} \dot{x}_{i}=0
$$

Wong's equation just says that the internal charge $Q^{I}$ is parallel-transported along the trajectory of a particle under the influence of the non-Abelian gauge field $A_{i}^{I}$. 
The geometrical meaning of Wong's equation, Eq. (246), can be seen as follows: Taking the total derivative of Eq. (244) with respect to time gives

$$
\left[\dot{x}_{i}, Q^{I}\right]=-\left[x^{i}, \dot{Q}^{I}\right]=\frac{i \hbar}{m} f_{J K}^{I} A_{i}^{J}(x, t) Q^{K} .
$$

This property can be used to show the formula for any field $\phi(x, t)=\phi^{I}(x, t) Q^{I} \in \mathcal{A}_{\hbar} \times \mathcal{G}$ :

$$
\begin{aligned}
{\left[\dot{x}_{i}, \phi^{I}(x, t) Q^{I}\right] } & =\left[\dot{x}_{i}, \phi^{I}(x, t)\right] Q^{I}+\phi^{I}(x, t)\left[\dot{x}_{i}, Q^{I}\right] \\
& =-\frac{i \hbar}{m}\left(\partial_{i} \phi^{I}+f^{I}{ }_{J K} A_{i}^{J} \phi^{K}\right) Q^{I} \\
& =-\frac{i \hbar}{m}\left(\partial_{i} \phi-\frac{i}{\hbar}\left[A_{i}, \phi\right]\right) \equiv-\frac{i \hbar}{m} D_{i} \phi .
\end{aligned}
$$

Recall that $p_{i}=m \dot{x}_{i}+A_{i}(x, t)$ are translation generators along $\mathbb{R}^{3}$, and remember the geometrical meaning of the Wong's equation, Eq. (246), stated above.

Now repeat Feynman's question: What is the most general interaction of a quantum particle carrying an internal charge satisfying Eq. (246) and the commutation relations in Eqs. (242)(244)? The calculation follows almost the same line [47] as that for the electromagnetic force except that the fields $E_{i}(x, t)=E_{i}^{I}(x, t) Q^{I} \in \mathcal{A}_{\hbar} \times \mathfrak{g}$ and $B_{i}(x, t)=B_{i}^{I}(x, t) Q^{I} \in \mathcal{A}_{\hbar} \times \mathfrak{g}$ now carry internal charges in the Lie algebra $\mathfrak{g}$; thus, Wong's equation, Eq. (246), has to be taken into account. We will not echo the derivation because it is almost straightforward with a careful Weyl-ordering. That may be a good exercise for graduate students.

The resulting force exerted on a quantum particle moving in $\mathbb{R}^{3} \times F$ is the generalized nonAbelian Lorentz force [47]

$$
F_{i}=E_{i}+\varepsilon^{i k l} \dot{x}_{k} B_{l}
$$

where the fields $E_{i}(x, t)=E_{i}^{I}(x, t) Q^{I}$ and $B_{i}(x, t)=B_{i}^{I}(x, t) Q^{I}$ satisfy

$$
\partial_{i} B_{i}-\frac{i}{\hbar}\left[A_{i}, B_{i}\right]=0, \quad \frac{\partial B_{i}}{\partial t}+\varepsilon^{i k l}\left(\partial_{k} E_{l}-\frac{i}{\hbar}\left[A_{k}, E_{l}\right]\right)=0
$$

The equations in Eq. (250), of course, can be summarized with the Lorentz covariant form (in the temporal gauge, $\left.A_{0}=0\right)$ as

$$
\varepsilon^{\mu \nu \rho \sigma} D_{\nu} F_{\rho \sigma}=0
$$

and the Bianchi identity, Eq. (251), can be solved by introducing non-Abelian gauge fields $A_{\mu}$ such that

$$
F_{\mu \nu}=\partial_{\mu} A_{\nu}-\partial_{\nu} A_{\mu}-\frac{i}{\hbar}\left[A_{\mu}, A_{\nu}\right]
$$


One can check the expression in Eq. (252). For example, one can get

$$
\varepsilon^{i k l} B_{l}^{I}=\partial_{i} A_{k}^{I}-\partial_{k} A_{i}^{I}+f_{J K}^{I} A_{i}^{J} A_{k}^{K}
$$

by using the Jacobi identity

$$
\left[Q^{I},\left[\dot{x}_{i}, \dot{x}_{k}\right]\right]+\left[\dot{x}_{i},\left[\dot{x}_{k}, Q^{I}\right]\right]+\left[\dot{x}_{k},\left[Q^{I}, \dot{x}_{i}\right]\right]=0
$$

together with Eqs. (238) and (247) where $B_{l}=B_{l}^{I} Q^{I}$.

\section{B. Symplectic Geometry Again}

The previous argument by Feynman clearly implies that the fundamental interactions such as electromagnetic, weak and strong forces can be understood as a symplectic or Poisson geometry of a particle phase space. Feynman starts with a very natural assumption about the Poisson structure of a particle interacting with external forces. In the case of a free particle, Eq. (228) is the well-known Heisenberg algebra: $\left[x^{i}, x^{k}\right]=0,\left[x^{i}, p_{k}\right]=i \hbar \delta_{k}^{i}$. (Note that Feynman and Dyson intentionally use $m \dot{x}_{i}$ instead of $p_{i}$.) If some external fields are turned on, then the particle momentum $m \dot{x}_{i}$ is no longer equal to $p_{i}$, but is shifted by $p_{i}-A_{i}$, where $A_{i}$ are arbitrary external fields. We easily see that, if the external fields $A_{i}$ depend only on $x$ and $t$, i.e., $A_{i}=A_{i}(x, t)$, and satisfy Wong's equation, Eq. (246), in the non-Abelian cases (to preserve the localizability in Eq. (244), the commutation relations, Eq. (228), remain intact, but that is not the whole story. We have repeatedly used the Jacobi identity of the algebra $\mathcal{A}_{\hbar}$ or $\mathcal{A}_{\hbar} \times \mathcal{G}$, which originally comes from the Poisson algebra in Eq. (4) of a particle phase space $P$ or $P \times F$. Recall that the Jacobi identity of Poisson bracket is not automatically guaranteed. The Schouten-Nijenhuis bracket for the Poisson tensor should vanish [77]. See Eq. (223). Therefore, the external fields $A_{i}$ cannot be completely arbitrary. They should not ruin the underlying Poisson structure. We know that, if the Poisson structure is nondegenerate, this condition is equivalent to the statement that the symplectic 2-form uniquely determined by the Poisson structure must be closed. See the discussion below Eq. (224). This is precisely the condition for gauge fields Feynman found. In gauge theory, it is called the Bianchi identity, e.g., $d F=0$ or $D F=0$.

There is an another beautiful observation [49] (orginally due to Jean-Marie Souriau) realizing Feynman's idea. Let $(P, \omega)$ be a symplectic manifold. One can properly choose local canonical coordinates $y^{a} \equiv\left(x^{1}, p_{1}, \cdots, x^{n}, p_{n}\right)$ in $P$ such that the symplectic structure $\omega$ can be written in 
the form

$$
\omega=\sum_{i=1}^{n} d x^{i} \wedge d p_{i} .
$$

Then, $\omega \in \Gamma\left(\Lambda^{2} T^{*} P\right)$ can be thought of as a bundle map $\omega: T P \rightarrow T^{*} P$. Because $\omega$ is nondegenerate at any point $y \in P$, we can invert this map to obtain the map $\eta \equiv \omega^{-1}: T^{*} P \rightarrow T P$. This cosymplectic structure $\eta=\frac{\partial}{\partial x^{i}} \wedge \frac{\partial}{\partial p_{i}} \in \omega \in \Gamma\left(\Lambda^{2} T P\right)$ is called the Poisson structure of $P$ and defines a Poisson bracket $\{\cdot, \cdot\}_{\hbar}$. See Section III.D. In a local chart with coordinates $y^{a}$, we have

$$
\{f, g\}_{\hbar}=\sum_{a, b=1}^{2 n} \eta^{a b} \frac{\partial f}{\partial y^{a}} \frac{\partial g}{\partial y^{b}} .
$$

Let $H: P \rightarrow \mathbb{R}$ be a smooth function on a Poisson manifold $(P, \eta)$. The vector field $X_{H}$ defined by $\iota_{X_{H}} \omega=d H$ is called a Hamiltonian vector field with the energy function $H$. We define a dynamical flow by using the differential equation [8]

$$
\frac{d f}{d t}=X_{H}(f)+\frac{\partial f}{\partial t}=\{f, H\}_{\hbar}+\frac{\partial f}{\partial t} .
$$

A solution to the above equation is a function $f$ such that for any path $\gamma:[0,1] \rightarrow M$, we have

$$
\frac{d f(\gamma(t))}{d t}=\{f, H\}_{\hbar}(\gamma(t))+\frac{\partial f(\gamma(t))}{\partial t} .
$$

The dynamics of a charged particle in an external static magnetic field is described by the Hamiltonian

$$
H=\frac{1}{2 m}(\mathbf{p}-e \mathbf{A})^{2}
$$

which is obtained from the free Hamiltonian $H_{0}=\frac{\mathbf{p}^{2}}{2 m}$ with the replacement

$$
\mathbf{p} \rightarrow \mathbf{p}-e \mathbf{A}
$$

Here, the electric charge of an electron is $q_{e}=-e$, and $e$ is a coupling constant identified with $g_{Y M}$. The symplectic structure in Eq. (255) leads to the Hamiltonian vector field $X_{H}$ given by

$$
X_{H}=\frac{\partial H}{\partial p_{i}} \frac{\partial}{\partial x^{i}}-\frac{\partial H}{\partial x^{i}} \frac{\partial}{\partial p_{i}} .
$$

Then, the Hamilton's equation, Eq. (257), reduces to the well-known Lorentz force law

$$
m \frac{d \mathbf{v}}{d t}=e \mathbf{v} \times \mathbf{B} .
$$


The observation in Ref. [49] (and of Jean-Marie Souriau) is that the Lorentz force law in Eq. (262) can be derived by keeping the Hamiltonian $H=H_{0}$, but instead shifting the symplectic structure

$$
\omega \rightarrow \omega^{\prime}=\omega-e B
$$

where $B=\frac{1}{2} B_{i k}(x) d x^{i} \wedge d x^{k}$. In this case, the Hamiltonian vector field $X_{H}$ is defined by $\iota_{X_{H}} \omega^{\prime}=$ $d H_{0}$ and given by

$$
X_{H}=\frac{\partial H_{0}}{\partial p_{i}} \frac{\partial}{\partial x^{i}}-\left(\frac{\partial H_{0}}{\partial x^{i}}-e B_{i k} \frac{\partial H_{0}}{\partial p_{k}}\right) \frac{\partial}{\partial p_{i}} .
$$

Then, one can easily check that the Hamilton's equation, Eq. (257), with the vector field in Eq. (264) reproduces the Lorentz force law in Eq. (262). Actually, one can show that the symplectic structure $\omega^{\prime}$ in Eq. (263) introduces a noncommutative phase space [9] such that the momentum space becomes noncommutative, i.e., $\left[p_{i}^{\prime}, p_{j}^{\prime}\right]=-i \hbar e B_{i j}$.

If a particle is interacting with electromagnetic fields, the influence of the magnetic field $B=$ $d A$ is described by 'minimal coupling', Eq. (260), and the new momenta $\mathbf{p}^{\prime}=-i \hbar\left(\nabla-i \frac{e}{\hbar} \mathbf{A}\right)$ are covariant under $U(1)$ gauge transformations. Let us point out that the minimal coupling can be understood as the Darboux transformation, Eq. (29), between $\omega$ and $\omega^{\prime}$. Consider the coordinate transformation $y^{a} \mapsto x^{a}(y)=\left(X^{1}, P_{1}, \cdots, X^{n}, P_{n}\right)(x, p)$ such that

$$
\sum_{i=1}^{n} d x^{i} \wedge d p_{i}=\sum_{i=1}^{n} d X^{i} \wedge d P_{i}-\frac{e}{2} \sum_{i, j=1}^{n} B_{i j}(X) d X^{i} \wedge d X^{j}
$$

with the Hamiltonian being unchanged, i.e., $H_{0}=\frac{\mathbf{P}^{2}}{2 m}$. The condition in Eq. (265) is equivalent to the following equations:

$$
\begin{aligned}
& \frac{\partial x^{i}}{\partial X^{j}} \frac{\partial p_{i}}{\partial X^{k}}-\frac{\partial x^{i}}{\partial X^{k}} \frac{\partial p_{i}}{\partial X^{j}}=-e B_{j k}, \\
& \frac{\partial x^{i}}{\partial X^{j}} \frac{\partial p_{i}}{\partial P_{k}}-\frac{\partial x^{i}}{\partial P_{j}} \frac{\partial p_{i}}{\partial X^{k}}=\delta_{j}^{k}, \\
& \frac{\partial x^{i}}{\partial P_{j}} \frac{\partial p_{i}}{\partial P_{k}}-\frac{\partial x^{i}}{\partial P_{k}} \frac{\partial p_{i}}{\partial P_{j}}=0 .
\end{aligned}
$$

The above equations are solved by

$$
x^{i}=X^{i}, \quad p_{i}=P_{i}+e A_{i}(X) .
$$

In summary the dynamics of a charged particle in an electromagnetic field has two equivalent descriptions [7]:

$$
\left(H=\frac{(\mathbf{p}-e \mathbf{A})^{2}}{2 m}, \omega\right)(x, p) \cong\left(H_{0}=\frac{\mathbf{P}^{2}}{2 m}, \omega^{\prime}=\omega-e B\right)(X, P) .
$$


The equivalence in Eq. (268) can easily be generalized to a time-dependent background $A^{\mu}=$ $\left(A^{0}, \mathbf{A}\right)(x, t)$ with the Hamiltonian $H=\frac{1}{2 m}(\mathbf{p}-e \mathbf{A})^{2}+e A^{0}$. The Hamilton's equation, Eq. (257), in this case is given by Eq. (230). The equivalence in Eq. (268) now means that the Lorentz force law, Eq. (230), can be obtained by using the Hamiltonian vector field in Eq. (264) with the Hamiltonian $H_{0}=\frac{\mathbf{p}^{2}}{2 m}+e A^{0}$ and noticing that the time dependence of the external fields now appears as the explicit $t$-dependence of momenta $p_{i}=p_{i}(t)$. Indeed, the electric field $\mathbf{E}$ appears as the combination $\mathbf{E}=-\nabla A^{0}+\frac{1}{e} \frac{\partial \mathbf{p}}{\partial t}$, but note that the coordinates $\left(x^{i}, p_{i}\right)$ in Eq. (264) correspond to $\left(X^{i}, P_{i}\right)$ in the notation of Eq. (265) and so $\frac{\partial \mathbf{p}}{\partial t}=-e \frac{\partial \mathbf{A}}{\partial t}$ by Eq. (267).

Feynman's approach transparently shows that electromagnetism is an inevitable structure in quantum particle dynamics and that we need an internal space (extra dimensions) to introduce nonAbelian forces. Furthermore, as emphasized by Dyson [46], Feynman's formulation also shows that nonrelativistic Newtonian mechanics and relativistic Maxwell's equations coexist peacefully. This is due to the underlying symplectic geometry as Souriau and Sternberg showed [49]. We know that the Lorentz force, Eq. (230), is generated by the minimal coupling $p_{\mu} \rightarrow \mathfrak{P}_{\mu} \equiv p_{\mu}-e A_{\mu}$ and that the minimal coupling can be encoded into the deformation of symplectic structure, which can be summarized as the relativistic form [80]: $\omega=-d \xi \rightarrow \omega^{\prime}=\omega-e F=-d(\xi+e A)$, where $\xi=\mathfrak{P}_{\mu} d x^{\mu}$ and $A=A_{\mu}(x) d x^{\mu}$. Therefore, the Maxwell equation $d F=0$ is simply interpreted as the closedness of the symplectic structure, and the minimal coupling is the Darboux transformation in Eq. (29) from the deformed symplectic structure $\omega^{\prime}=\omega-e F$, as was shown in Eq. (265). In this symplectic formulation of particle dynamics, the gauge symmetry defined by $A \rightarrow A+d \lambda$ is actually symplectomorphisms, i.e., diffeomorphisms generated by Hamiltonian vector fields $X_{\lambda}$ satisfying $\mathcal{L}_{X_{\lambda}} \omega=0$. In this sense, the gauge symmetry is derived from the symplectic or Poisson geometry, so one may regard the underlying symplectic or Poisson structure as a more fundamental structure of particle dynamics. Also, one may notice a great similarity between the symplectic geometries of particles and spacetime geometry (gravity).

A symplectic formulation of the equations of motion of a particle was generalized to a YangMills field by Sternberg in [49] and Weinstein in [81]. Let $\pi: P \rightarrow M$ be a principal $G$-bundle, and let $F$ be a Hamiltonian $G$-space. This means that $F$ is a symplectic manifold with symplectic form $\Omega$ such that $G$ acts on $F$ as a group of symplectic diffeomorphisms so that there is a homomorphism of the Lie algebra $\mathfrak{g}$ of $G$ into the algebra of Hamiltonian vector fields and that we are given a lifting of this homomorphism to a homomorphism of $\mathfrak{g}$ into the Lie algebra of functions on $F$, where the Lie algebra structure is given by Poisson bracket. Thus, to each $\xi \in \mathfrak{g}$, we get a 
function $f_{\xi}$ on $F$ and a Hamiltonian vector field $\xi_{F}$ on $F$ so that $\iota_{\xi_{F}} \Omega=-d f_{\xi}$.

Let $E \subset T^{*} M \times P$ be the pull-back of $P$ by the canonical projection $\widetilde{\pi}: T^{*} M \rightarrow M$, i.e., the following diagram commutes:

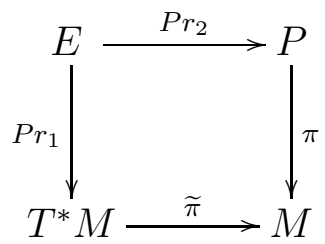

Sternberg shows [49] how a connection on $P$ can be used to put a symplectic structure on the associated bundle $E \times_{G} F \rightarrow T^{*} M$ with fiber $F$. Given a Hamiltonian function $H: T^{*} M \rightarrow \mathbb{R}$, one may pull it back to $E \times_{G} F$ and thereby obtain a Hamiltonian flow that represents the motion of a classical particle under the influence of the field for which the given connection is a YangMills field. That is, every connection on a principal bundle $P$ induces a Poisson structure on the associated bundle $E \times_{G} F$. The resulting symplectic mechanics of a particle in a Yang-Mills field is actually equivalent to Feynman's approach in Section IV.A. More details in terms of the local formula will be discussed elsewhere.

\section{Emergent Matters from Stable Geometries}

Now let us pose our original problem about what matter is in emergent geometry. We speculated that particles or matter fields may be realized as a topological object in a noncommutative $\star-$ algebra $\mathcal{A}_{\theta}$ and, thus, as a stable localized state in a Hilbert space $\mathcal{H}$, e.g., the Fock space (18). If so, we can assign the concept of positions and velocities (as collective variables) to these localized states such that they satisfy some well-defined (quantum) Poisson algebra, e.g., Eqs. (242)-(244), which are inherited from the original noncommutative $\star$-algebra $\mathcal{A}_{\theta}$. Here, we will suggest a plausible picture based on the Fermi-surface scenario in Refs. [82, 83], but we will not insist on our proposal.

Particles are by definition characterized by their positions and momenta, besides their intrinsic charges, e.g., spin, isospin and an electric charge. They should be replaced by matter fields in relativistic quantum theory in order to incorporate pair creation and pair annihilation. Moreover, in a noncommutative space such as Eq. (17), the very notion of a point is replaced by a state in the Hilbert space of Eq. (18); thus, the concept of particles (and matter fields, too) becomes ambiguous, so the following question should be meaningful and addressed: What is the most natural notion of a particle or a corresponding matter field in the noncommutative $\star$-algebra of Eq. 
(19)? We suggested in Ref. [7] that it should be a K-theory object in the sense of Ref. [82].

We consider the $U(N)$ Yang-Mills theory described by the action in Eq. (151) defined on a $d$-dimensional Minkowski spacetime $\mathbb{R}_{C}^{d}$. As we explained in Section III.C, the theory in Eq. (151) can be related to both $U(N)$ Yang-Mills theories and noncommutative $U(1)$ gauge theories in various dimensions and different $B$-field backgrounds by applying the matrix T-duality in Eq. (188) and the correspondence in Eq. (22). Thereby, we will assume that the $U(N)$ Yang-Mills theory in Eq. (151) has been obtained from the BFSS matrix model in Eq. (190) by using the $(d-1)$-fold matrix T-duality in Eq. (188). In particular, it will be important to remember that the $U(N \rightarrow \infty)$ gauge theory in Eq. (151) in the Moyal background in Eq. (152) can be mapped to the $D=d+2 n$-dimensional noncommutative $U(1)$ gauge theory in Eq. (161).

Motivated by this fact, we will specify our problem as follows: We want to classify a stable class of "time-independent solutions" in the action in Eq. (161) satisfying the asymptotic boundary condition in Eq. (152). For such kind of solutions, we may simply forget about time and work in the temporal gauge, $A_{0}=0$. Therefore, we will consider the $U(N)$ gauge-Higgs system $\left(\mathbf{A}, \Phi^{a}\right)(\mathbf{x})$ as a map from $\mathbb{R}_{C}^{p}$ to $G L(N, \mathbb{C})$, where $p \equiv d-1$ and $z^{\mu}=(t, \mathbf{x})$. As long as we require the fields in the theory to approach the common limit in Eq. (152) (which does not depend on $\mathbf{x}$ ) as $\mathbf{x} \rightarrow \infty$ in any direction, we can think of $\mathbb{R}^{p}$ as having the topology of a sphere $\mathbf{S}^{p}=\mathbb{R}^{p} \cup\{\infty\}$, with the point at infinity being included as an ordinary point.

Note that the matrices $\Phi^{a}(\mathbf{x})(a=1, \cdots, 2 n)$ are nondegenerate along $\mathbf{S}^{p}$ because we have assumed Eq. (152). Therefore, $\Phi^{a}$ defines a well-defined map [82]

$$
\Phi^{a}: \mathbf{S}^{p} \rightarrow G L(N, \mathbb{C})
$$

from $\mathbf{S}^{p}$ to the group of nondegenerate complex $N \times N$ matrices. If this map represents a nontrivial class in the $p$ th homotopy group $\pi_{p}(G L(N, \mathbb{C}))$, the solution in Eq. (269) will be stable under small perturbations, and the corresponding nontrivial element of $\pi_{p}(G L(N, \mathbb{C}))$ represents a topological invariant. Note that the map in Eq. (269) is contractible to the group of maps from $\mathbf{S}^{p}$ to $U(N)$ [52].

If we think of $G L(N, \mathbb{C})$ as an endomorphism from $\mathbb{C}^{N}$ to itself, $\mathbb{C}^{N}$ is already big enough to embed $\mathbf{S}^{p}$ into it if $N>p / 2$. This leads to a remarkable point that there is the so-called stable regime at $N>p / 2$, where $\pi_{p}(G L(N, \mathbb{C}))$ is independent of $N$. In this stable regime, the homotopy groups of $G L(N, \mathbb{C})$ or $U(N)$ define a generalized cohomology theory, known as Ktheory [50-53]. In K-theory, which also involves vector bundles and gauge fields, any smooth 
manifold $X$ is assigned an Abelian group $K(X)$. Aside from a deep relation to D-brane charges and RR fields in string theory [51, 52], the K-theory is also deeply connected with the theory of Dirac operators, the index theorem, Riemannian geometry, noncommutative geometry, etc. [14].

The matrix action in Eq. (151) describes a $U(N \rightarrow \infty)$ vector (Chan-Paton) bundle supported on $\mathbb{R}_{C}^{d}$. The homotopy map in Eq. (269) is to classify stable solutions of the $U(N)$ Chan-Paton bundle that cannot be dissipated by small perturbations. However, the topological classification should be defined up to pair creations and pair annihilations because there is no way to suppress such quantum effects. This is the reason [52, 53] $K(X)$ is the right answer for classifying the topological class of excitations in the $U(N)$ gauge-Higgs system. For $X$ noncompact, $K(X)$ is to be interpreted as compact K-theory [50]. For example, for $X=\mathbb{R}^{d}$, this group is given by

$$
K\left(\mathbb{R}^{d}\right)=\pi_{d-1}(G L(N, \mathbb{C}))
$$

with $N$ in the stable regime. The corresponding groups are known to exhibit Bott periodicity such that $K\left(\mathbb{R}^{d}\right)=\mathbf{Z}$ for even $d$ and $K\left(\mathbb{R}^{d}\right)=0$ for odd $d$.

With the above understanding, let us find an explicit construction of a topologically non-trivial excitation. It is well-known [53] that this can be done using an elegant construction due to Atiyah, Bott and Shapiro (ABS) [54]. The construction uses the gamma matrices of the Lorentz group $S O(p, 1)$ for $X=\mathbb{R}_{C}^{d}$ to construct explicit generators of the K-theory group in Eq. (270), where $p=d-1$. Let $X$ be even dimensional so that $K(X)=\mathbf{Z}$, and $S_{ \pm}$be two irreducible spinor representations of $\operatorname{Spin}(d)$ Lorentz group, and $p_{\mu}=(\omega, \mathbf{p}), \mu=0,1, \cdots, p$, be the momenta along $X$. We define the gamma matrices $\Gamma^{\mu}: S_{+} \rightarrow S_{-}$of $S O(p, 1)$ obeying $\left\{\Gamma^{\mu}, \Gamma^{\nu}\right\}=2 \eta^{\mu \nu}$. Also, we introduce an operator $\mathcal{D}: \mathcal{H} \times S_{+} \rightarrow \mathcal{H} \times S_{-}$[82] such that

$$
\mathcal{D}=\Gamma^{\mu} p_{\mu}+\cdots
$$

which is regarded as a linear operator acting on a Hilbert space $\mathcal{H}$, as well as the spinor vector space $S_{ \pm}$. Here, the Hilbert space $\mathcal{H}$ is possibly much smaller than the Fock space in Eq. (18), because the Dirac operator in Eq. (271) acts on collective (coarse-grained) modes of the solution in Eq. (269).

The ABS construction implies [82, 83] that the Dirac operator in Eq. (271) is a generator of $\pi_{p}(U(N))$ as a nontrivial topology in momentum space $(\mathbf{p}, \omega)$ and acts on a low lying excitation near the vacuum in Eq. (152) which carries K-theory charges and so is stable. Such modes are described by using coarse-grained fermions $\chi^{A}(\omega, \mathbf{p}, \theta)$, with $\theta$ denoting possible collective 
coordinates of the solution in Eq. (269) [82]. The ABS construction determines the range $\widetilde{N}$ of the index $A$ carried by the coarse-grained fermions $\chi^{A}$ to be $\widetilde{N}=2^{[p / 2]} n \leq N$ complex components. The precise form of the fermion $\chi^{A}$ depends on its K-theory charge, whose explicit representation on $\mathcal{H} \times S_{ \pm}$will be given later. Feynman's approach [46] in Section IV.A will provide a clearcut picture to see what the multiplicity $n$ means. At low energies, the dispersion relation of the fermion $\chi^{A}$ is given by the relativistic Dirac equation

$$
i \Gamma^{\mu} \partial_{\mu} \chi+\cdots=0
$$

with possible gauge interactions and higher order corrections in higher energies. Thus, we get a spinor of the Lorentz group $S O(p, 1)$ from the ABS construction as a topological solution in momentum space [82]. For example, in four dimensions, i.e., $p=3, \chi^{A}$ has two complex components when $n=1$, so it describes a chiral Weyl fermion.

Although the emergence of $(p+1)$-dimensional spinors is just a consequence due to the fact that the ABS construction uses the Clifford algebra to construct explicit generators of $\pi_{p}(U(N))$, its physical origin is mysterious and difficult to understand. However, we believe that the coherent spactime vacuum in Eq. (17) would be the crux for the origin of the fermionic nature of particles and the mysterious connection between the Clifford module and K-theory [54]. An important future problem would be to clearly understand this issue.

Now, let us address the problem to determine the multiplicity $n$ of the coarse-grained fermions $\chi^{\alpha a}$, where we decomposed the index $A=(\alpha a)$ with $\alpha$ the spinor index of the $S O(d)$ Lorentz group and $a=1, \cdots, n$ an internal index of an $n$-dimensional representation of some compact symmetry $G$. In order to understand this problem, we will identify the noncommutative $\star$-algebra $\mathcal{A}_{\theta}$ with $G L(N, \mathbb{C})$ by using the relation in Eq. (22). Under this correspondence, the $U(N \rightarrow$ $\infty)$ gauge theory in Eq. (151) in the Moyal background in Eq. (152) can be mapped to the $D$-dimensional noncommutative $U(1)$ gauge theory in Eq. (161) defined on $\mathbb{R}_{C}^{d} \times \mathbb{R}_{N C}^{2 n}$ where $D=d+2 n$. Then, the K-theory in Eq. (270) for any sufficiently large $N$ can be identified with the K-theory $K\left(\mathcal{A}_{\theta}\right)$ for the noncommutative $\star$-algebra $\mathcal{A}_{\theta}$ [52].

As we showed in Section III.B, the generic fluctuation in Eq. (163) will deform the background spacetime lattice defined by the Fock space in Eq. (18), which generates gravitational fields given by the metric in Eq. (169). For simplicity, we will consider low-energy excitations around the solution in Eq. (269) whose K-theory class is given by $K\left(\mathcal{A}_{\theta}\right)$. In this case, the solution in Eq. (269) would be a sufficiently localized state described by a compact (bounded self-adjoint) 
operator in $\mathcal{A}_{\theta}$. This means that it does not appreciably disturb the ambient gravitational field. Therefore, we may reduce the problem to quantum particle dynamics on $X \times F$ [7], where $X=\mathbb{R}_{C}^{d}$ and $F$ is an internal space describing collective modes of the solution in Eq. (269). It is natural to identify the coordinates of $F$ with an internal charge of $G$ carried by the fermion $\chi^{\alpha a}$. To be specific, the (collective) coordinates of $F$ will take values in the Lie algebra $\mathfrak{g}$ of $G$, such as the isospins or colors, and will be denoted by $Q^{I}\left(I=1, \cdots, n^{2}-1\right)$. In the end, we essentially revisit Feynman's problem, which we addressed in Section IV.A.

The quantum particle dynamics on $X \times F$ naturally requires the introduction of non-Abelian gauge fields in the representation of the Lie algebra in Eq. (243), and the dynamics of the particle carrying an internal charge in $F$ will be defined by a symplectic structure on $T^{*} X \times F$. Note that $\mathbb{R}_{N C}^{2 n}$ already has its symplectic structure $B=\frac{1}{2} B_{a b} d y^{a} \wedge d y^{b}$, originated from the noncommutative space in Eq. (154). Also, note that the action in Eq. (161) has only $U(1)$ gauge fields on $\mathbb{R}_{C}^{d} \times \mathbb{R}_{N C}^{2 n}$, so the problem is how to get the Lie algebra generators in Eq. (243) from the space $\mathbb{R}_{N C}^{2 n}$ and how to get the non-Abelian gauge fields $A_{\mu}^{I}(z) \in \mathfrak{g}$ on $X$ from the $U(1)$ gauge fields on $\mathbb{R}_{C}^{d} \times \mathbb{R}_{N C}^{2 n}$. Here, it is enough to consider only the transverse gauge fields $A_{\mu}^{I}(z)$ as low-lying excitations because the solution in Eq. (269) is actually coming from the longitudinal gauge field $\widehat{A}_{a}(z, y)$ in Eq. (157).

The problem is solved [7] by noting that the $n$-dimensional harmonic oscillator in quantum mechanics can realize $S U(n)$ symmetries (see the Chapter 14 in Ref. [84]). The generators of the $S U(n)$ symmetry on the Fock space in Eq. (18) are given by

$$
Q^{I}=a_{i}^{\dagger} T_{i k}^{I} a_{k}
$$

where the creation and the annihilation operators are given by Eq. (17) and the $T^{I}$, s are constant $n \times n$ matrices satisfying $\left[T^{I}, T^{J}\right]=i f^{I J}{ }_{K} T^{K}$ with the same structure constants as Eq. (243) . It is easy to check that the $Q^{I}$ 's satisfy the $S U(n)$ Lie algebra (243). We introduce the number operator $Q^{0} \equiv a_{i}^{\dagger} a_{i}$ and identify it with a $U(1)$ generator. The operator $\mathfrak{C}=\sum_{I} Q^{I} Q^{I}$ is the quadratic Casimir operator of the $S U(n)$ Lie algebra and commutes with all $Q^{I}$ 's. Thus, one may identify $\mathfrak{C}$ with an additional $U(1)$ generator.

Let $\rho(\mathcal{H})$ be a representation of the Lie algebra in Eq. (243) in a Hilbert space $\mathcal{H}$. We take an $n$-dimensional representation in $\mathcal{H}=L^{2}\left(\mathbb{C}^{n}\right)$, a square integrable Hilbert space. Because the solution in Eq. (269) is described by a compact operator in $\mathcal{A}_{\theta}$, its representation space $\mathcal{H}=$ $L^{2}\left(\mathbb{C}^{n}\right)$ will be much smaller (with finite basis in generic cases) than the original Fock space in 
Eq. (18). Thus, let us expand the $U(1)$ gauge field $\widehat{A}_{\mu}(z, y)$ in Eq. (157) with the $S U(n)$ basis in Eq. (273):

$$
\begin{aligned}
\widehat{A}_{\mu}(z, y) & =\sum_{n=0}^{\infty} \sum_{I_{i} \in \rho(\mathcal{H})} A_{\mu}^{I_{1} \cdots I_{n}}\left(z, \rho, \lambda_{n}\right) Q^{I_{1}} \cdots Q^{I_{n}} \\
& =A_{\mu}(z)+A_{\mu}^{I}\left(z, \rho, \lambda_{1}\right) Q^{I}+A_{\mu}^{I J}\left(z, \rho, \lambda_{2}\right) Q^{I} Q^{J}+\cdots
\end{aligned}
$$

where $\rho$ and $\lambda_{n}$ are eigenvalues of $Q^{0}$ and $\mathfrak{C}$, respectively, in the representation $\rho(\mathcal{H})$. The expansion in Eq. (274) is formal, but it is assumed that each term in Eq. (274) belongs to the irreducible representation of $\rho(\mathcal{H})$. Through the expansion in Eq. (274), we get $S U(n)$ gauge fields $A_{\mu}^{I}(z)$, as well as $U(1)$ gauge fields $A_{\mu}(z)$, as low-lying excitations [7].

Note that the coarse-grained fermion $\chi$ in Eq. (272) behaves as a stable relativistic particle in the spacetime $X=\mathbb{R}_{C}^{d}$. When these fermionic excitations are given, there will also be bosonic excitations arising from changing the position along $X$ of the internal charge $F$. According to Feynman's picture, especially Wong's equation, Eq. (246), the gauge fields in Eq. (274) represent collective modes for the position change in $X=\mathbb{R}_{C}^{d}$ of the charge $F$ [83]. See Eq. (248) for the geometrical interpretation of Wong's equation, Eq. (246). Thus, they can be regarded as collective modes in the vicinity of an internal charge living in $F$ and interact with the fermions in Eq. (272).

Therefore, we think of the Dirac operator, Eq. (271), as an operator $\mathcal{D}: \mathcal{H} \times S_{+} \rightarrow \mathcal{H} \times S_{-}$, where $\mathcal{H}=L^{2}\left(\mathbb{C}^{n}\right)$, and we introduce a minimal coupling with the $U(1)$ and $S U(n)$ gauge fields in Eq. (274) by the replacement $p_{\mu} \rightarrow p_{\mu}-e A_{\mu}-A_{\mu}^{I} Q^{I}$. Then, the Dirac equation, Eq. (272), becomes

$$
i \Gamma^{\mu}\left(\partial_{\mu}-i e A_{\mu}-i A_{\mu}^{I} Q^{I}\right) \chi+\cdots=0
$$

Here, we see that the coarse-grained fermion $\chi$ in the homotopy class $\pi_{p}(U(N))$ is in the fundamental representation of $S U(n)$, so we identify the multiplicity $n$ in the ABS construction in Eq. (272) with the number of colors in $S U(n)$ [7].

The most interesting case in Eq. (161) is of $d=4$ and $n=3$, that is, 10-dimensional noncommutative $U(1)$ gauge theory on $\mathbb{R}_{C}^{4} \times \mathbb{R}_{N C}^{6}$. In this case, Eq. (275) is the 4-dimensional Dirac equation, where $\chi$ is a quark, an $S U(3)$ multiplet of chiral Weyl fermions, which couples with gluons $A_{\mu}^{I}(z), S U(3)$ gauge fields for the color charge $Q^{I}$, as well as photons $A_{\mu}(z), U(1)$ gauge fields for the electric charge $e$. One may consider a similar ABS construction in the vector space $\mathbb{C}^{2} \times \mathbb{C} \subset \mathbb{C}^{3}$, i.e., by breaking the $S U(3)$ symmetry into $S U(2) \times U(1)$, where $\chi$ would be a 
lepton, an $S U(2)$ doublet of chiral Weyl fermions coupling with $S U(2)$ gauge fields. In this case, $Q^{I}(I=1,2,3)$ in Eq. (273) are the famous Schwinger representation of the $S U(2)$ Lie algebra.

To conclude, we may go back to our starting point. Our starting point was the $d$-dimensional $U(N)$ Yang-Mills theory defined by the action in Eq. (151) or equivalently $D$-dimensional noncommutative $U(1)$ gauge theory defined by the action in Eq. (161). We observed that the theory in Eq. (151) allows topologically stable solutions as long as the homotopy group in Eq. (269) is nontrivial, and we argued that a matter field, such as leptons and quarks, simply arises from such a stable solution and that non-Abelian gauge fields correspond to collective zero-modes of the stable localized solution. Although we intended to interpret such excitations as particles and gauge fields and to ignore their gravitational effects, we have to remember that these are originally a part of spacetime geometry according to the map in Eq. (163). Consequently, we get a remarkable picture, if any, that matter fields, such as leptons and quarks, simply arise as a stable localized geometry, which is a topological object in the defining algebra (noncommutative $\star$-algebra) of quantum gravity.

\section{ANATOMY OF SPACETIME}

It is in order to discuss the most beautiful aspects of emergent gravity. Remarkably, the emergent gravity reveals a novel picture about the origin of spacetime, dubbed as emergent spacetime, which is radically different from any previous physical theory all of which describe what happens in a given spacetime. Thus, we may take it for granted that emergent gravity leads to many results that are radically different from Einstein gravity.

\section{A. Emergent Time in Emergent Gravity}

We have intentionally postponed posing the formidable issue how "Time" emerges, together with space, and how it is entangled with space to unfold into a single entity, spacetime, and take the shape of Lorentz covariance. Now we are ready to address this formidable issue.

Let $(M, \pi)$ be a Poisson manifold. We previously defined the anchor map $\pi^{\sharp}: T^{*} M \rightarrow T M$ in Eq. (224) for a general Poisson bivector $\pi \in \Gamma\left(\Lambda^{2} T M\right)$ and the Hamiltonian vector field in Eq. (225) by

$$
X_{H} \equiv-\pi^{\sharp}(d H)=\{\cdot, H\}_{\pi}=\pi^{\mu \nu}(x) \frac{\partial H}{\partial x^{\nu}} \frac{\partial}{\partial x^{\mu}},
$$


where $H: M \rightarrow \mathbb{R}$ is a smooth function on the Poisson manifold $(M, \pi)$. If the Poisson tensor $\pi$ is nondegenerate so that $\pi^{-1} \equiv \omega \in \Gamma\left(\Lambda^{2} T^{*} M\right)$ is a symplectic structure on $M$, the anchor map $\pi^{\sharp}: T^{*} M \rightarrow T M$ defines a bundle isomorphism because $\pi^{\sharp}$ is nondegenerate everywhere. We will speak of the flow $\phi_{t}$ of a vector field $X$ on $M$ when referring to a 1-parameter group of diffeomorphisms generated by $X$.

Any Poisson manifold $(M, \pi)$ always admits a Hamiltonian dynamical system on $M$ defined by a Hamiltonian vector field $X_{H}$ and it is described by

$$
\frac{d f}{d t}=X_{H}(f)+\frac{\partial f}{\partial t}=\{f, H\}_{\pi}+\frac{\partial f}{\partial t}
$$

for any $f \in C^{\infty}(\mathbb{R} \times M)$. If $\phi_{t}$ is a flow generated by a Hamiltonian vector field $X_{H}$, the following identity holds [8]:

$$
\begin{aligned}
\frac{d}{d t}\left(f \circ \phi_{t}\right) & =\frac{d}{d t} \phi_{t}^{*} f=\phi_{t}^{*} \mathcal{L}_{X_{H}} f+\phi_{t}^{*} \frac{\partial f}{\partial t} \\
& =\phi_{t}^{*}\{f, H\}_{\pi}+\frac{\partial f}{\partial t} \circ \phi_{t} \\
& =\left(\{f, H\}_{\pi}+\frac{\partial f}{\partial t}\right) \circ \phi_{t} .
\end{aligned}
$$

Thus, we can get $f(x, t)=g\left(\phi_{t}(x)\right)$, where $g(x) \equiv f(x, 0)$. If $\pi=\eta=\frac{\partial}{\partial x^{i}} \wedge \frac{\partial}{\partial p_{i}}$, we precisely reproduce Eqs. (257) and (258) from Eqs. (277) and (278), respectively. In this case, the evolution of a particle system is described by the dynamical flow in Eq. (278) generated by the Hamiltonian vector field in Eq. (276) for a given Hamiltonian $H$.

Introduce an extended Poisson tensor on $\mathbb{R} \times M[8]$

$$
\tilde{\pi}=\pi+\frac{\partial}{\partial t} \wedge \frac{\partial}{\partial H}
$$

and a generalized Hamiltonian vector field

$$
\widetilde{X}_{H} \equiv-\widetilde{\pi}^{\sharp}(d H)=\{\cdot, H\}_{\widetilde{\pi}}=\pi^{\mu \nu}(x) \frac{\partial H}{\partial x^{\nu}} \frac{\partial}{\partial x^{\mu}}+\frac{\partial}{\partial t} .
$$

We can then rewrite Hamilton's equation, Eq. (277), compactly in the form

$$
\frac{d f}{d t}=\widetilde{X}_{H}(f)=\{f, H\}_{\widetilde{\pi}}=\{f, H\}_{\pi}+\frac{\partial f}{\partial t} .
$$

Similarly, we can extend the symplectic structure $\omega=\pi^{-1}$ to the product manifold $\mathbb{R} \times M$ by considering a new symplectic structure $\widetilde{\omega}=\pi_{2}^{*} \omega$, where $\pi_{2}: \mathbb{R} \times M \rightarrow M$ is the projection such that $\pi_{2}(t, x)=x$. Define $\omega_{H}=\widetilde{\omega}+d H \wedge d t$. Then, the pair $\left(\mathbb{R} \times M, \omega_{H}\right)$ is called a contact manifold [8]. 
Suppose that observables $f \in C^{\infty}(M)$ do not depend on time explicitly, i.e., $\frac{\partial f}{\partial t}=0$. Look at Eq. (278). We understand that the time evolution of the system in this case is determined by simply calculating the Poisson bracket with a Hamiltonian function $H$. In other words, in the case of $\frac{\partial f}{\partial t}=0$, the time evolution is just the inner automorphism of the Poisson algebra $\left(M,\{\cdot, \cdot\}_{\pi}\right)$ [85]. Therefore, time in Hamilton's equation, Eq. (277), is basically an affine parameter to trace the history of a particle, and it is operationally defined by the Hamiltonian. That is, time in Hamiltonian dynamics is intrinsically the histories of the particles themselves. However, we have to notice that, only when the symplectic structure is fixed for a given Hamiltonian, the evolution of the system is completely determined by the evolution equation in Eq. (278). In this case, the dynamics of the system can be formulated in terms of an evolution with a single time parameter. In other words, we have a globally well-defined time for the evolution of the system. This is the usual situation we consider in classical mechanics.

If observables $f \in C^{\infty}(M)$ including the Hamiltonian $H$, explicitly depend on time, i.e., $\frac{\partial f}{\partial t} \neq 0$, the time evolution of the system is not completely determined by the inner automorphism of the Poisson algebra only, so the time evolution partially becomes an outer automorphism. However, as we remarked above, we can extend an underlying Poisson structure as in Eq. (279) or introduce a contact manifold $\left(\mathbb{R} \times M, \omega_{H}\right)$ by extending an underlying symplectic structure. The time evolution of a particle system is again defined by an inner automorphism of the extended Poisson algebra $\left(\mathbb{R} \times M,\{\cdot, \cdot\}_{\tilde{\pi}}\right)$. In this case, time should be regarded as a dynamical variable whose conjugate momentum is given by the Hamiltonian $H$, as indicated by the Poisson structure in Eq. (279). Thus, the time should be defined locally in this case. Let us clarify this situation.

Consider a dynamical evolution described by a change of a symplectic structure from $\omega$ to $\omega_{t}=\omega+t\left(\omega^{\prime}-\omega\right)$ for all $0 \leq t \leq 1$, where $\omega^{\prime}-\omega=-e d A$. The Moser lemma, Eq. (27), says that there always exists a one-parameter family of diffeomorphisms generated by a smooth time-dependent vector field $X_{t}$ satisfying $\iota_{X} \omega_{t}=e A$. Although the vector field $X_{t}$ defines a dynamical one-parameter flow, the vector field $X_{t}$ is, in general, not even locally Hamiltonian because $d A \neq 0$. The evolution of the system in this case is locally described by the flow $\phi_{t}$ of $X_{t}$ starting at $F_{0}=$ identity, but it is no longer a (locally) Hamiltonian flow. In this case, we fail to have the property $\mathcal{L}_{X_{t}} f=\{f, H\}_{\pi}$ in Eq. (278), so we have no global Hamiltonian flow. That is, there is no well-defined or global time for the particle system. In other words, the time flow $\phi_{t}$ of $X_{t}$ is defined on a local chart and describes only a local evolution of the system.

We observed the equivalence in Eq. (268) for the dynamics of a charged particle. Let us 
consider the above situation by looking at the left-hand side picture of Eq. (268) by fixing the symplectic structure, but instead by changing the Hamiltonian. (Note that the magnetic field in the Lorentz force, Eq. (262), does not do any work, so there is no energy flow during the evolution.) At time $t=0$, the system is described by the free Hamiltonian $H_{0}$, but it ends up with the Hamiltonian in Eq. (259) at time $t=1$. Therefore, the dynamics of the system cannot be described with a single time parameter covering the entire period $0 \leq t \leq 1$. We can introduce at most a local time during $\delta t<\epsilon$ on a local patch and smoothly adjust to a neighboring patch. To say, a clock of the particle will tick each time with a different rate because the Hamiltonian of the particle is changing during time evolution. As we already remarked before, we may also need to quantize the time according to the Poisson structure in Eq. (279) in order to describe a quantum evolution of a system in terms of an extended inner automorphism such as that in Eq. (281).

Now, we can apply the same philosophy to the case of the Poisson structure in Eq. (6) defined on a space itself [7]. The mathematics is exactly the same. An essential point in defining the time evolution of a system was that any Poisson manifold $(M, \pi)$ always admits the Hamiltonian dynamical system in Eq. (277) on $M$ defined by the Hamiltonian vector field $X_{H}$ given by Eq. (276). We have faced the same situation with the $\theta$-bracket, Eq. (6), whose time evolution was summarized in Eq. (27). Of course, one should avoid a confusion between the dynamical evolution of a particle system related to the phase space in Eq. (4) and the dynamical evolution of spacetime geometry related to the noncommutative space in Eq. (6).

We learn an important lesson from Souriau and Sternberg [49] that the Hamiltonian dynamics in the presence of electromagnetic fields can be described by the deformation of a symplectic structure of a phase space. More precisely, we observed that the emergent geometry is defined by a one-parameter family of diffeomorphisms generated by a smooth vector field $X_{t}$ satisfying $\iota_{X} \omega_{t}+A=0$ for the change of a symplectic structure within the same cohomology class from $\omega$ to $\omega_{t}=\omega+t\left(\omega^{\prime}-\omega\right)$ for all $0 \leq t \leq 1$ where $\omega^{\prime}-\omega=d A$. The vector field $X_{t}$ is, in general, not a Hamiltonian flow, so no global time can be assigned to the evolution of the symplectic structure $\omega_{t}$. However, if there is no fluctuation of the symplectic structure, i.e., $F=d A=0$ or $A=-d H$, there can be a globally well-defined Hamiltonian flow. In this case, we can define a global time by introducing a unique Hamiltonian such that the time evolution is defined by $d f / d t=X_{H}(f)=\{f, H\}_{\theta=\omega^{-1}}$ everywhere. In particular, when the initial symplectic structure $\omega$ is constant (homogeneous), a clock will tick everywhere at the same rate. Note that this situation happens for the constant background in Eq. (17) from which a flat spacetime emerges as we will 
discuss soon in some detail. If $\omega$ is not constant, the time evolution will not be uniform over space and a clock will tick at different rates at different places. This is consistent with Einstein gravity because a nonconstant $\omega$ corresponds to a curved space in our picture, as we explained in Section III.D.

In the case of a changing symplectic structure, we can apply the same strategy as we did in the particle case with the Poisson structure $\pi=\theta$, so we suggest, in general, the concept of "Time" in emergent gravity [7] as a contact manifold $\left(\mathbb{R} \times M, \omega_{H}\right)$, where $(M, \omega)$ is a symplectic manifold and $\omega_{H}=\widetilde{\omega}+d H \wedge d t$, with $\widetilde{\omega}=\pi_{2}^{*} \omega$ defined by the projection $\pi_{2}: \mathbb{R} \times M \rightarrow M, \pi_{2}(t, x)=x$. A question is then how to recover the (local) Lorentz symmetry in the end. As we pointed out above, if $(M, \omega)$ is a canonical symplectic manifold, i.e., $M=\mathbb{R}^{2 n}$ and $\omega=$ constant, a $(2 n+1)$ dimensional Lorentz symmetry appears from the contact manifold $\left(\mathbb{R} \times M, \omega_{H}\right)$. (For a more general case such as our $(3+1)$-dimensional Lorentzian world and a Poisson spacetime, Eq. (223), we may instead use the Poisson structure in Eqs. (279)-(281), or we may need an even more general argument, which we don't know yet.) Once again, the Darboux theorem says that there always exists a local coordinate system in which the symplectic structure has a canonical form. See Eq. (29). For the Poisson case, we can apply Weinstein's splitting theorem instead. Then, it is quite plausible that the Lorentz symmetry on a local Darboux chart would be recovered in a local way. Furthermore, Feynman's argument in Section IV.A implies that the gauge symmetry, as well as the Lorentz symmetry, is just derived from the symplectic structure on the contact manifold $\left(\mathbb{R} \times M, \omega_{H}\right)$. For example, one can recover the gauge symmetry along the time direction by defining the Hamiltonian $H=A_{0}+H^{\prime}$ and the time evolution of a spacetime geometry by the Hamilton's equation $D_{0} f \equiv d f / d t+\left\{A_{0}, f\right\}_{\widetilde{\theta}=\widetilde{\omega}^{-1}}=\left\{f, H^{\prime}\right\}_{\widetilde{\theta}=\widetilde{\omega}^{-1}}$. Then, one may interpret Hamilton's equation as an infinitesimal version of an inner automorphism like (163), which was, indeed, used to define the vector field $V_{0}(X)$ in Eq. (164).

Our proposal for the emergent time [7] is based on the fact that a symplectic manifold $(M, \omega)$ always admits a Hamiltonian dynamical system on $M$ defined by a Hamiltonian vector field $X_{H}$, i.e., $\iota_{X_{H}} \omega=d H$. The emergent time can be generalized to the noncommutative space in Eq. (8) by considering the inner derivation, Eq. (144), instead of the Poisson bracket $\{f, H\}_{\theta}$. If time is emergent in this way, it implies a very interesting consequence. Note that every symplectic manifold $(M, B)$ is canonically oriented and comes with a canonical measure, the Liouville measure, $B^{n}=\frac{1}{n !} B \wedge \cdots \wedge B$, which is a volume form of the symplectic manifold $(M, B)$ and nowhere vanishing on $M$. Therefore, the symplectic structure $B$ triggered by the vacuum condensate in Eq. 
(198) not only causes the emergence of spacetime but also specifies an orientation of spacetime. Because the time evolution of spacetime is defined by the Poisson structure $\pi=\theta=B^{-1}$ as in Eq. (278), a global time evolution of spacetime manifold will have a direction that depends on the orientation $B^{n}$, although a local time evolution has time reversal symmetry. If gravity is emergent from the electromagnetism supported on a symplectic manifold as we have envisaged so far, it may also be possible to explain the "arrow of time" in the cosmic evolution of our Universe - the most notoriously difficult problem in quantum gravity.

\section{B. Cosmological Constant Problem and Dark Energy}

In general relativity, gravitation arises out of the dynamics of spacetime being curved by the presence of stress-energy, and the equations of motion for the metric fields of spacetime are determined by the distribution of matter and energy:

$$
R_{\mu \nu}-\frac{1}{2} g_{\mu \nu} R=\frac{8 \pi G}{c^{4}} T_{\mu \nu}
$$

The Einstein equations, Eq. (282), describe how the geometry of spacetime on the left-hand side is determined dynamically in harmony with matter fields on the right-hand side, at first sight. We know that the existence of spacetime leads to a "metrical elasticity" of space, i.e., to an inertial force that opposes the curving of space.

However, there is a deep conflict between the spacetime geometry described by general relativity and the matter fields described by quantum field theory [86]. If spacetime is flat, i.e., $g_{\mu \nu}=\eta_{\mu \nu}$, the left-hand side of Eq. (282) identically vanishes, so the energy-momentum tensor of matter fields should vanish, i.e., $T_{\mu \nu}=0$. In other words, a flat spacetime is completely empty with no energy. Thus, the concept of empty space in Einstein gravity is in an acute contrast to the concept of vacuum in quantum field theory, where the vacuum is not empty but is full of quantum fluctuations. As a result, a vacuum is extremely heavy, and its weight is on the order of the Planck mass, i.e., $\rho_{\mathrm{vac}} \sim M_{P}^{4}$.

The conflict rises to the surface that gravity and matters respond differently to the vacuum energy and perplexingly brings about the notorious cosmological constant problem. Indeed, the clash manifests itself as a mismatch of symmetry between gravity and matter [87]. To be precise, if we shift a matter Lagrangian $\mathcal{L}_{M}$ by a constant $\Lambda$, that is,

$$
\mathcal{L}_{M} \rightarrow \mathcal{L}_{M}-2 \Lambda
$$


it results in a shift of the matter energy-momentum tensor by $T_{\mu \nu} \rightarrow T_{\mu \nu}-\Lambda g_{\mu \nu}$ in the Einstein equation, Eq. (282), although the equations of motion for matter are invariant under the shift in Eq. (283). Definitely the $\Lambda$-term in Eq. (283) will appear as the cosmological constant in Einstein gravity, and it affects the spacetime structure. For example, a flat spacetime is no longer a solution of Eq. (282).

Let us sharpen the problem arising from the conflict between geometry and matter. In quantum field theory, there is no way to suppress quantum fluctuations in a vacuum. Fortunately, the vacuum energy due to the quantum fluctuations, regardless of how large they are, does not cause any trouble for quantum field theory thanks to the symmetry in Eq. (283). However, general covariance requires that gravity couple universally to all kinds of energy. Therefore, the vacuum energy $\rho_{\text {vac }} \sim M_{P}^{4}$ will induce a highly-curved spacetime whose curvature scale $R$ would be $\sim M_{P}^{2}$ according to Eq. (282). If so, the quantum field theory framework in the background of quantum fluctuations must be broken down due to a large back-reaction of background spacetim, but we know that it is not the case. The quantum field theory is well-defined, even in the presence of the vacuum energy $\rho_{\text {vac }} \sim M_{P}^{4}$, and the background spacetime still remains flat, as we empirically know. So far, there is no experimental evidence for the vacuum energy really coupling to gravity, although it is believed that the vacuum energy is real as experimentally verified by the Casimir effect.

Which side of Eq. (282) is the culprit giving rise to the incompatibility? After consolidating all the suspicions inferred above, we throw doubt on the left-hand side of Eq. (282), especially, on the result that a flat spacetime is free gratis, i.e., costs no energy. We should remark that such a result is not compatible with the inflation scenario either because it implies that a huge vacuum energy in a highly nonequilibrium state is required to generate an extremely large spacetime. Note that Einstein gravity is not completely background independent because it assumes the prior existence of a spacetime manifold. Here, we refer to a background-independent theory in which no spacetime structure is a priori assumed, but is defined by the theory. In particular, the flat spacetime is a geometry of special relativity rather than general relativity, and so it is assumed to be a priori given without reference to its dynamical origin. This reasoning implies that the negligence about the dynamical origin of a flat spacetime defining a local inertial frame in general relativity might be the core root of the incompatibility inherent in Eq. (282).

All in all, one may be tempted to infer that a flat spacetime may not be free gratis, but a result of Planck energy condensation in a vacuum. Now, we will show that inference to be true 
[41]. Surprisingly, the emergent spacetime picture then appears as the Hóly Gráil to cure several notorious problems in theoretical physics; for example, the cosmological constant problem, the nature of dark energy and the reason gravity is so weak compared to other forces. After all, our final destination is to check whether the emergent gravity from noncommutative geometry is a physically viable theory to correctly explain the dynamical origin of flat spacetime.

Let us start with a background-independent matrix theory, for example, Eqs. (133) or (189), where no spacetime structure is introduced. A specific spacetime background, e.g., a flat spacetime, has been defined by specifying the vacuum, Eq. (152), of the theory. Now, look at the metric in Eq. (89) or in Eq. (169) to trace back to where the flat spacetime comes from. The flat spacetime is the case with $V_{a}^{\mu}=\delta_{a}^{\mu}$, so $\lambda^{2}=1$. The vector field $V_{a}=V_{a}^{\mu} \partial_{\mu}=\partial_{a}$ in this case comes from the noncommutative gauge field $A_{a}^{(0)} \equiv\left\langle\widehat{A}_{a}^{(0)}\right\rangle_{\mathrm{vac}}=-B_{a b} y^{b}$ in Eq. (199) whose field strength is $\left\langle\widehat{F}_{a b}^{(0)}\right\rangle_{\text {vac }}=-B_{a b}$, describing a uniform condensation of gauge fields in vacuum. See Eq. (198). Therefore, we see that the flat spacetime is emergent from the vacuum algebra in Eq. (8) induced by a uniform condensation of gauge fields in vacuum. This is a tangible difference from Einstein gravity in which the flat spacetime is completely an empty space.

The emergent gravity defined by the action in Eq. (161), for example, responds completely differently to the constant shift in Eq. (283). To be specific, let us consider a constant shift of the background $B_{M N} \rightarrow B_{M N}+\delta B_{M N}$. Then, the action in Eq. (161) in the new background becomes

$$
\widehat{S}_{B+\delta B}=\widehat{S}_{B}+\frac{1}{2 g_{Y M}^{2}} \int d^{D} X \widehat{F}_{M N} \delta B_{M N}-\frac{1}{4 g_{Y M}^{2}} \int d^{D} X\left(\delta B_{M N}^{2}-2 B^{M N} \delta B_{M N}\right) .
$$

The last term in Eq. (284) is simply a constant; thus, it will not affect the equations of motion, Eq. (179). The second term is a total derivative, so it will vanish if $\int d^{D} X \widehat{F}_{M N}=0$. (It is a defining property [9] in the definition of a star product that $\int d^{D} X \widehat{f} \star \widehat{g}=\int d^{D} X \widehat{f} \cdot \widehat{g}$. Then, the second term should vanish as far as $\widehat{A}_{M} \rightarrow 0$ at infinity.) If spacetime has a nontrivial boundary, the second term could be nonvanishing at the boundary, which would change the theory under the shift. We will not consider a nontrivial spacetime boundary because the boundary term is not an essential issue here, though there should be interesting physics at the boundary [88]. Then we get the result $\widehat{S}_{B+\delta B} \cong \widehat{S}_{B}$. Indeed, this is the Seiberg-Witten equivalence between noncommutative field theories defined by the noncommutativity $\theta^{\prime}=\frac{1}{B+\delta B}$ and $\theta=\frac{1}{B}$ [16]. Although the vacuum in Eq. (198) readjusts itself under the shift, the Hilbert spaces $\mathcal{H}_{\theta^{\prime}}$ and $\mathcal{H}_{\theta}$ in Eq. (18) are completely isomorphic if and only if $\theta$ and $\theta^{\prime}$ are nondegenerate constants. Furthermore, the vector fields in 
Eq. (163) generated by $B+\delta B$ and $B$ backgrounds are equally flat as long as they are constant. Consequently two different constant backgrounds are related by a global Lorentz transformation. Equation (121) also shows that the background gauge field does not contribute to the energymomentum tensor in Eq. (125).

Therefore, we clearly see that a constant shift of energy density such as Eq. (283) is a symmetry of the theory in Eq. (161) although the action in Eq. (161) defines a theory of gravity in the sense of emergent gravity. As a consequence, there is no cosmological constant problem in emergent gravity [41]. Now, let us estimate the dynamical scale of the vacuum condensation in Eq. (198). Because gravity emerges from noncommutative gauge fields, the parameters $g_{Y M}^{2}$ and $|\theta|$ defining a noncommutative gauge theory should be related to the Newton constant $G$ in emergent gravity. A simple dimensional analysis leads to the relation in Eq. (127). This relation immediately leads to the fact [7] that the energy density of the vacuum in Eq. (198) is

$$
\rho_{\mathrm{vac}} \sim\left|B_{a b}\right|^{2} \sim M_{P}^{4},
$$

where $M_{P}=(8 \pi G)^{-1 / 2} \sim 10^{18} \mathrm{GeV}$ is the Planck mass. Therefore, the emergent gravity finally reveals a remarkable picture that the huge Planck energy $M_{P}$ is actually the origin of the flat spacetime. Hence, we conclude that a vacuum energy does not gravitate differently from Einstein gravity, and a flat spacetime is not free gratis, but is a result of Planck energy condensation in vacuum [41].

If the vacuum algebra in Eq. (8) describes a flat spacetime, it can have a very important implication to cosmology. According to our picture for emergent spacetime, a flat spacetime is emergent from Planck energy condensation in vacuum; thus, the time scale for the condensate will be roughly on the order of the Planck time. We know that there was an epoch of very violent timevarying vacuum, the so-called cosmic inflation. Therefore, it is natural to expect that the explosive inflation era that lasted roughly $10^{-33}$ seconds at the beginning of our Universe corresponds to a dynamical process enormously spreading out a flat spacetime by the instantaneous condensation of vacuum energy $\rho_{\text {vac }} \sim M_{P}^{4}$. Unfortunately, it is not clear how to microscopically describe this dynamical process by using the matrix action (189). Nevertheless, it is quite obvious that the cosmological inflation should be a dynamical condensation of the vacuum energy $\rho_{\text {vac }} \sim M_{P}^{4}$ for the generation of (flat) spacetime according to our emergent gravity picture.

In addition, our picture for the emergent spacetime implies that the global Lorentz symmetry should be a perfect symmetry up to the Planck energy because the flat spacetime was emergent 
from Planck energy condensation in vacuum - the maximum energy in Nature. The huge vacuum energy $\rho_{\mathrm{vac}} \sim\left|B_{a b}\right|^{2} \sim M_{P}^{4}$ was simply used to make a flat spacetime and, surprisingly, does not gravitate [41]! Then, the gravitational fields generated by the deformations of the background in Eq. (198) will be very weak because the spacetime vacuum is very solid with a stiffness of the Planck scale. Hence the dynamical origin of flat spacetime is intimately related to the weakness of the gravitational force. Furthermore, the vacuum algebra in Eq. (17) describes an extremely coherent condensation because it is equal to the Heisenberg algebra of an $n$-dimensional quantum harmonic oscillator. As a consequence, the noncommutative algebra (17) should describe a zeroentropy state in spite of the involvement of the Planck energy. This is very mysterious, but it should be the case because a flat spacetime emergent from the algebra in Eq. (17) is completely an empty space from the viewpoint of Einstein gravity and, so, has no entropy. This reasoning also implies that the condensation of vacuum energy $\rho_{\mathrm{vac}} \sim M_{P}^{4}$ happened at most once.

We observed that the dynamical scale of the vacuum condensate is on the order of the Planck scale. The emergence of spacetime was caused by Planck energy accumulating in vacuum, but the Planck energy condensation causes the underlying spacetime to be noncommutative, which will introduce an uncertainty relation between microscopic spacetimes. Therefore, a further accumulation of energy over the noncommutative spacetime will be subject to UV/IR mixing [89]. UV/IR mixing in noncommutative spacetime then implies that any UV fluctuations on the order of the Planck scale $L_{P}$ will be necessarily paired with IR fluctuations of a typical scale $L_{H}$. These vacuum fluctuations around the flat spacetime will add a tiny energy $\delta \rho$ to the vacuum in Eq. (285) so that the total energy density is equal to $\rho \sim M_{P}^{4}+\delta \rho$. A simple dimensional analysis and a symmetry consideration, e.g., the cosmological principle, lead to the following estimate of the vacuum fluctuation [87]:

$$
\rho=\rho_{\mathrm{vac}}+\delta \rho \sim M_{P}^{4}\left(1+\frac{L_{P}^{2}}{L_{H}^{2}}\right)=M_{P}^{4}+\frac{1}{L_{P}^{2} L_{H}^{2}} .
$$

It might be remarked that, though the second term in Eq. (286) is nearly constant within a Hubble patch, it is not completely constant over the entire spacetime while the first term is a true constant because the vacuum fluctuation $\delta \rho$ has a finite size of $L_{H}$, so it will act as a source of spacetime curvature of the order of $1 / L_{H}^{2}$. Because the first term in $\rho$ does not gravitate, the second term $\delta \rho$ will, thus, be a leading contributor to the deformation of the global spacetime curvature, leading possibly to a de Sitter phase. Interestingly, this energy of vacuum fluctuations, $\delta \rho \sim \frac{1}{L_{P}^{2} L_{H}^{2}}$, is in good agreement with the observed value of current dark energy [41, 87] if $L_{H}$ is identified with 
the size of the cosmic horizon of our universe.

As we reasoned above, the existence of the energy $\delta \rho$ in Eq. (286) seems to be a generic property of emergent gravity based on a noncommutative spacetime. Therefore, the emergent spacetime would leave the vestige of this energy everywhere. Readers may remember that we discussed some strange energy in Section II.C, so let us go back to Eq. (124). Although we have taken the Euclidean signature to get the result in Eq. (124), we will simply assume that it can be analytically continued to the Lorentzian signature. The Wick rotation will be defined by $y^{4}=i y^{0}$. Under this Wick rotation, $\delta_{a b} \rightarrow \eta_{a b}=(-+++)$ and $\varepsilon^{1234}=1 \rightarrow-\varepsilon^{0123}=-1$. Then, we get $\Psi_{a}^{(E)}=i \Psi_{a}^{(L)}$ according to the definition in Eq. (118). It is then given by [7]

$$
T_{\mu \nu}^{(L)}=\frac{1}{16 \pi G_{4} \lambda^{2}}\left(\rho_{\mu} \rho_{\nu}+\Psi_{\mu} \Psi_{\nu}-\frac{1}{2} g_{\mu \nu}\left(\rho_{\lambda}^{2}+\Psi_{\lambda}^{2}\right)\right)
$$

where $\rho_{\mu}=2 \partial_{\mu} \lambda$ and $\Psi_{\mu}=E_{\mu}^{a} \Psi_{a}$

The Raychaudhuri equation [90, 91] represents the evolution equations of the expansion, shear and rotation of flow lines along the flow generated by a vector field in a background spacetime. Here, we introduce an affine parameter $\tau$ labeling points on the curve of the flow. Given a timelike unit vector field $u^{\mu}$, i.e., $u^{\mu} u_{\mu}=-1$, the Raychaudhuri equation in four dimensions is given by

$$
\dot{\Theta}-\dot{u}_{; \mu}^{\mu}+\Sigma_{\mu \nu} \Sigma^{\mu \nu}-\Omega_{\mu \nu} \Omega^{\mu \nu}+\frac{1}{3} \Theta^{2}=-R_{\mu \nu} u^{\mu} u^{\nu}
$$

$\Theta=u_{; \nu}^{\mu}$ represents the expansion/contraction of volume and $\dot{\Theta}=\frac{d \Theta}{d \tau}$ while $\dot{u}^{\mu}=u_{; \nu}^{\mu} u^{\nu}$ represents the acceleration due to nongravitational forces, e.g., the Lorentz force. $\Sigma_{\mu \nu}$ and $\Omega_{\mu \nu}$ are the shear tensor and the vorticity tensor, respectively, which are all orthogonal to $u^{\mu}$, i.e., $\Sigma_{\mu \nu} u^{\nu}=\Omega_{\mu \nu} u^{\nu}=0$. The Einstein equation, Eq. (125), can be rewritten as

$$
R_{\mu \nu}=8 \pi G\left(T_{\mu \nu}-\frac{1}{2} g_{\mu \nu} T_{\lambda}^{\lambda}\right)
$$

where $T_{\mu \nu}=E_{\mu}^{a} E_{\nu}^{b} T_{a b}$. One can see from Eq. (289) that the right-hand side of Eq. (288) is given by

$$
-R_{\mu \nu} u^{\mu} u^{\nu}=-\frac{1}{2 \lambda^{2}} u^{\mu} u^{\nu}\left(\rho_{\mu} \rho_{\nu}+\Psi_{\mu} \Psi_{\nu}\right)
$$

where we have considered the energy-momentum tensor, Eq. (287), only for simplicity.

Suppose that all the terms on the left-hand side of Eq. (288), except the expansion evolution $\dot{\Theta}$, vanish or become negligible. In this case, the Raychaudhuri equation reduces to

$$
\dot{\Theta}=-\frac{1}{2 \lambda^{2}} u^{\mu} u^{\nu}\left(\rho_{\mu} \rho_{\nu}+\Psi_{\mu} \Psi_{\nu}\right)
$$


Note that the Ricci scalar is given by $R=\frac{1}{2 \lambda^{2}} g^{\mu \nu}\left(\rho_{\mu} \rho_{\nu}+\Psi_{\mu} \Psi_{\nu}\right)$. Therefore, $R<0$ when $\rho_{\mu}$ and $\Psi_{\mu}$ are timelike while $R>0$ when they are spacelike. Remember that our metric signature is $(-+++)$, so, for timelike perturbations, $\dot{\Theta}<0$, which means that the volume of a three-dimensional spacelike hypersurface orthogonal to $u_{\mu}$ decreases. However, if spacelike perturbations are dominant, the volume of the three-dimensional spacelike hypersurface can expand. For example, consider the scalar perturbations in Eq. (129), i.e.,

$$
\left\langle\rho_{a} \rho_{b}\right\rangle=\frac{1}{4} \eta_{a b} \rho_{c}^{2}, \quad\left\langle\Psi_{a} \Psi_{b}\right\rangle=\frac{1}{4} \eta_{a b} \Psi_{c}^{2}
$$

For spacelike purturbations, Eq. (291) becomes

$$
\dot{\Theta}=\frac{R}{4}>0
$$

The perturbation in Eq. (292) does not violate the energy condition because $u^{\mu} u^{\nu} T_{\mu \nu}^{(L)}=\frac{R}{32 \pi G}>0$ according to Eq. (287). This means that the Liouville energy-momentum tensor in Eq. (287) can act as a source of gravitational repulsion and exert a negative pressure causing an expansion of the universe, possibly leading to a de Sitter phase [91]. As was pointed out in Eq. (130), it can behave like a cosmological constant, i.e., $\rho=-p$, in a constant (or almost constant) curvature spacetime. Another important property is that the Liouville energy in Eq. (287) is vanishing for the flat spacetime, so it should be small if spacetime is not so curved.

To be more quantitative, let us consider the fluctuation in Eq. (292) and look at the energy density $u^{\mu} u^{\nu} T_{\mu \nu}^{(L)}$ along the flow represented by a timelike unit vector $u^{\mu}$ as in Eq. (290). Note that the Riemannian volume is given by $\nu_{g}=\lambda^{2} \nu=\sqrt{-g} d^{4} y$. Also, it was shown in Ref. [7] that $\Psi_{\mu}$ is the Hodge-dual to the 3-form $H$. Thus, $u^{\mu} \rho_{\mu}$ and $u^{\mu} \Psi_{\mu}$ refer to the volume change of a three-dimensional spacelike hypersurface orthogonal to $u^{\mu}$. Assume that the radius of the threedimensional hypersurface is $L_{H}(\tau)$ at time $\tau$, where $\tau$ is an affine parameter labeling the curve of the flow. Then, it is reasonable to expect that $u^{\mu} \rho_{\mu}=2 u^{\mu} \partial_{\mu} \lambda \approx 2 \lambda / L_{H}(\tau) \approx u^{\mu} \Psi_{\mu}$ because the Ricci scalar $R \sim \frac{1}{L_{H}^{2}}$. After all, we approximately get [7]

$$
u^{\mu} u^{\nu} T_{\mu \nu}^{(L)} \sim \frac{1}{8 \pi G L_{H}^{2}}=\frac{1}{L_{P}^{2} L_{H}^{2}} .
$$

If we identify the radius $L_{H}$ with the size of cosmic horizon, the energy density in Eq. (294) reproduces the dark energy $\delta \rho$ in Eq. (286) up to a factor. 


\section{CONCLUSION}

We suggested that the quantum gravity must be defined by quantizing spacetime itself by the Newton constant $G$. This quantization scheme is very different from the conventional one in which quantization basically quantizes an infinite-dimensional particle phase space associated with spacetime metric fields in terms of the Planck constant $\hbar$. Our observation is that the existence of the Newton constant in Nature can be translated into a symplectic or Poisson structure of spacetime and that the canonical quantization of the underlying symplectic or Poisson structure inevitably leads to spacetime noncommutative geometry. It turns out that electromagnetism defined on the symplectic or Poisson spacetime enjoys very beautiful properties: the Darboux theorem and the Moser lemma. From these theorems, we can formulate the equivalence principle even for the electromagnetic force such that there always exists a coordinate transformation to locally eliminate the electromagnetic force. This equivalence principle can be fully lifted to a noncommutative spacetime; thus, the so-called "quantum equivalence principle" can be identified with a gauge equivalence between star products. This implies that quantum gravity can consistently be derived from the quantum equivalence principle and that matter fields can arise from the quantized spacetime.

If gravity emerges from a field theory, it is necessary to realize the Newton constant $G$ from the field theory. That is the reason the field theory should be defined with an intrinsic parameter of (length) $)^{2}$, and a noncommutative spacetime elegantly carries out this mission. The only other example of such a theory carrying an intrinsic constant of (length $)^{2}$ is string theory in which $\alpha^{\prime}$ plays the role of $G$ or $|\theta|$. A unique feature of string theory due to the existence of $\alpha^{\prime}$ is Tduality [11], which is a symmetry between small and large distances, symbolically represented by $R \leftrightarrow \alpha^{\prime} / R$. This symmetry implies the existence of a minimum length scale in spactime and signifies an intrinsic noncommutative spacetime geometry. The T-duality is a crucial ingredient for various string dualities and mirror symmetry. For the very similar reason, gravity in string theory also basically arises in the context of emergent gravity although many string theorists seem to be reluctant to accept this interpretation. Recently, Blau and Theisen vividly summarized this picture in their review article [92]:

There are basically two approaches to formulate a quantum theory of gravity. The first treats gravity as a fundamental interaction which it attempts to quantise. In the second approach gravity is not fundamental but an emergent phenomenon. String theory 
falls into the second category. It has the gratifying feature that not only gravity but also the gauge interactions which are mediated by a spin one gauge boson are emergent. String theory thus provides a unifying framework of all elementary particles and their interactions: it inevitably and automatically includes gravity (in the form of a massless traceless symmetric second-rank tensor excitation of the closed string, identified with the graviton) in addition to gauge forces which arise from massless excitation of the open or closed string (depending on the perturbative formulation of the theory).

We think the emergent gravity we have discussed so far is very parallel to string theory in many aspects. We may understand this wonderful similarity by noticing the following fact [7]: A Riemannian geometry is defined by a pair $(M, g)$, where the metric $g$ encodes all geometric information, while a symplectic geometry is defined by a pair $(M, \omega)$, where the 2 -form $\omega$ encodes all. A basic concept in Riemannian geometry is a distance defined by the metric. One may identify this distance with a geodesic worldline of a "particle" moving in $M$. On the contrary, a basic concept in symplectic geometry is an area defined by the symplectic structure. One may regard this area as a minimal worldsheet swept by a "string" moving in $M$. In this picture, the wiggly string, so a fluctuating worldsheet, may be interpreted as a deformation of the symplectic structure in spacetime $M$. Then, we know that a Riemannian geometry (or gravity) is emergent from wiggly strings or the deformation of the symplectic structure! Amusingly, the Riemannian geometry is probed by particles while the symplectic geometry would be probed by strings.

Hence the emergent gravity we have reviewed in this paper may be deeply related to string theory. This may be supported by the fact that many essential aspects of string theory, for example, AdS/CFT correspondence, open-closed string duality, noncommutative geometry, mirror symmetry, etc. have also been realized in the context of emergent noncommutative geometry. Thus, we may moderately claim that string theory is simply a "stringy" realization of symplectic or Poisson spacetime.

There are many important issues that we didn't even touch on. Although we have speculated that matter fields can emerge from stable localized geometries defined by noncommutative $\star$-algebra, we could not understand how particle masses can be generated from the noncommutative $\star$-algebra, in other words, how to realize spontaneous electroweak symmetry breaking or the Higgs mechanism. We believe this problem could be deeply related to the question of how the 
extra internal space $F$ for weak and strong forces in Section IV is dynamically compactified. We don't know this yet even though we have some vague ideas. Thus, from the background independent formulation of quantum gravity, the Standard Model is completely unexplored territory. The emergent spacetime picture may present a radically new understanding of the Standard Model.

We have no idea how supersymmetry arises from a background-independent quantum gravity theory or what the role of supersymmetry is in the emergent geometry and emergent matter. We do not know how to break it, but this issue should be understood in the near future.

Though we have tried to concretely formulate emergent gravity as much as possible, a rigorous mathematical formulation of emergent gravity, especially background-independent quantum gravity, is highly demanded. We think that the Lie algebroid may be a useful mathematical framework for emergent gravity. Here, we will introduce the definition of a Lie algebroid [77] only to appreciate some flavor of its mathematical structure for emergent quantum gravity. Progress along this line will be published elsewhere.

A Lie algebroid is a triple $(E,[\cdot, \cdot], \rho)$ consisting of a smooth vector bundle $E$ over a manifold $M$, together with a Lie algebra structure $[\cdot, \cdot]$ on the vector space $\Gamma(E)$ of the smooth global sections of $E$, and a morphism of vector bundles $\rho: E \rightarrow T M$, called the anchor map, where $T M$ is the tangent bundle of $M$. The anchor map and the bracket satisfy the Leibniz rule such that

$$
[X, f Y]=f[X, Y]+(\rho(X) f) \cdot Y
$$

for all $X, Y \in \Gamma(E)$ and $f \in C^{\infty}(M)$. Here, $\rho(X) f$ is the derivative of $f$ along the vector field $\rho(X)$. The anchor $\rho$ defines a Lie algebra homomorphism from the Lie algebra of sections of $E$, with Lie bracket $[\cdot, \cdot]$, into the Lie algebra of vector fields on $M$, i.e.,

$$
\rho([X, Y])=[\rho(X), \rho(Y)]
$$

If $M$ is a Poisson manifold, then the cotangent bundle $T^{*} M \rightarrow M$ is, in a natural way, a Lie algebroid over $M$. The anchor is the map $\pi^{\sharp}: T^{*} M \rightarrow T M$ defined by the Poisson bivector $\pi$. See Eq. (224). The Lie bracket $[\cdot, \cdot]$ of differential 1-forms satisifes $[d f, d g]=d\{f, g\}_{\pi}$ for any functions $f, g \in C^{\infty}(M)$, where $\{f, g\}_{\pi}=\pi(d f, d g)$ is the Poisson bracket defined by $\pi$. When $\pi$ is nondegenerate, $M$ is a symplectic manifold, and this Lie algebra structure of $\Gamma\left(T^{*} M\right)$ is isomorphic to that of $\Gamma(T M)$. A noncommutative generalization, i.e. $\{f, g\}_{\pi} \rightarrow-i[\widehat{f}, \widehat{g}]_{\star}$, seems to be possible.

Because background-independent quantum gravity does not assume any kind of spacetime 
structure, a natural question is then why spacetime on large scales is four dimensions. If gravity is emergent from gauge field interactions, we may notice that electromagnetism is now only a long-range force in Nature. Weak and strong forces are short-range forces, so they will affect only microscopic structure of spacetime. Then, we may infer that only electromagnetism is responsible for the large-scale structure of spacetime. In this regard, there is a funny coincidence [40]. If we compare the number of physical polarizations of photons and gravitons in $D$ dimensions and find the matching condition of the physical polarizations, we get a cute number: $\mathbf{\Psi}\left(A_{\mu}\right)=D-2=\frac{D(D-3)}{2}=\boldsymbol{\Psi}\left(g_{\mu \nu}\right) \Rightarrow D=1$ or $D=4$, where denotes the number of polarizations. Of course, we have to throw $D=1$ away because it is not physically meaningful. Does this unfledged math have some meaning?

\section{Acknowledgments}

This work was supported by the Daejin University Research Grants 2014. This work was partly done when HSY visited the KEK Theory Center using the International Visitor Program. He thanks Yoshihisa Kitazawa and Jun Nishimura for warm hospitality and helpful discussions during his visit and Koji Hashimoto for enlightening discussion at RIKEN theory seminar.

[1] Nevertheless, gravitational phenomena are ubiquitous in our everyday life. The reason is that the gravitational force is only attractive and always additive. As a result, the standard gravitational parameter $G M$ for an astronomical body with mass $M$ is not small. For example, $G M_{e}=4 \times 10^{14} \mathrm{~m}^{3} / \mathrm{s}^{2}$ for the Earth, where $M_{e}=5.96 \times 10^{24} \mathrm{Kg}$, which corresponds to $1 \mathrm{~cm}$ compared to the Planck length $L_{p l}=\sqrt{G} \sim 10^{-33} \mathrm{~cm}$.

[2] Niels Henrik Abel (1802-1829): A problem that seems insurmountable is just seemingly so because we have not asked the right question. You should always ask the right question and then you can solve the problem.

[3] C. W. Misner, K. S. Thorne and J. A. Wheeler, Gravitation (W. H. Freeman and Company, New York, 1973).

[4] H. S. Yang, Europhys. Lett. 88, 31002 (2009), hep-th/0608013.

[5] H. S. Yang, Int. J. Mod. Phys. A 24, 4473 (2009), hep-th/ 0611174. 
[6] H. S. Yang, Eur. Phys. J. C 64, 445 (2009), arXiv: 0704.0929.

[7] H. S. Yang, J. High Energy Phys. 05, 012 (2009), arXiv: 0809.4728.

[8] R. Abraham and J. E. Marsden, Foundations of Mechanics (Addison-Wesley, Reading, 1978).

[9] M. R. Douglas and N. A. Nekrasov, Rev. Mod. Phys. 73, 977 (2001), hep-th/0106048, R. J. Szabo, Phys. Rep. 378, 207 (2003), hep-th/0109162.

[10] R. J. Szabo, Class. Quantum Grav. 23, R199 (2006), hep-th/0606233

[11] K. Becker, M. Becker and J. Schwarz, String Theory and M-Theory: A Modern Introduction (Cambridge University Press, New York, 2007).

[12] There is an interesting peculiarity in $D=6$ that the gauge coupling constant $e^{2}$ in natural units $c=\hbar=1$ carries the physical dimension of (length) $)^{2}$. Thus, the gauge coupling constant $e^{2}$ in six dimensions operates like a noncommutative parameter $|\theta|$ or a string length $\alpha^{\prime}$, i.e., $e^{2} \sim|\theta| \sim \alpha^{\prime}$. This may imply that a six-dimensional gauge theory intrinsically behaves like a string theory without assuming any noncommutative spacetime.

[13] The theory on a D-brane also needs an intrinsic length parameter because it is always defined with the action $\int_{M} \sqrt{\operatorname{det}\left(g+2 \pi \alpha^{\prime} F\right)}$, so $\alpha^{\prime} \equiv l_{s}^{2}$ should carry the dimension of (length) ${ }^{2}$ for a dimensional reason. Because the parameter $l_{s}^{2}$ disappears from the theory if $F=0$, it is necessary for the field strength $F$ to be nowhere vanishing in order for the parameter $l_{s}^{2}$ to have an operational meaning as an intrinsic length scale of the theory. In this case where $\langle F\rangle_{r \rightarrow \infty} \equiv B$, the two theories will be physically equivalent, as was shown in Ref. [16].

[14] A. Connes, Noncommutative Geometry (Academic Press, San Diego, CA, 1994).

[15] M. Kontsevich, Lett. Math. Phys. 66, 157 (2003), q-alg/9709040.

[16] N. Seiberg and E. Witten, J. High Energy Phys. 09, 032 (1999), hep-th/9908142.

[17] T. Banks, W. Fischler, S. H. Shenker and L. Susskind, Phys. Rev. D 55, 5112 (1997), hep-th/9610043.

[18] N. Ishibashi, H. Kawai, Y. Kitazawa and A. Tsuchiya, Nucl. Phys. B 498, 467 (1997), hep-th/9612115; H. Aoki, S. Iso, H. Kawai, Y. Kitazawa and T. Tada, Prog. Theor. Phys. 99, 713 (1998), hep-th/9802085; H. Aoki, N. Ishibashi, S. Iso, H. Kawai, Y. Kitazawa and T. Tada, Nucl. Phys. B 565, 176 (2000), hep-th/9908141.

[19] L. Motl, hep-th/9701025; R. Dijkgraaf, E. Verlinde and H. Verlinde, Nucl. Phys. B 500, 43 (1997), hep-th/9703030.

[20] W. Taylor, Rev. Mod. Phys. 73, 419 (2001), hep-th/0101126. 
[21] G. E. Volovik, The Universe in a Helium Droplet (Oxford University Press, Oxford, 2003); C. Barcelo, S. Liberati and M. Visser, Living Rev. Rel. 8, 12 (2005), gr-qc/0505065

[22] J. M. Maldacena, Adv. Theor. Math. Phys. 2, 231 (1998), hep-th/9711200; S. S. Gubser, I. R. Klebanov and A. M. Polyakov, Phys. Lett. B 428, 105 (1998), hep-th/9802109; E. Witten, Adv. Theor. Math. Phys. 2, 253 (1998), hep-th/9802150.

[23] V. O. Rivelles, Phys. Lett. B 558, 191 (2003), hep-th/0212262.

[24] H. S. Yang, Mod. Phys. Lett. A 21, 2637 (2006), hep-th/0402002; R. Banerjee and H. S. Yang, Nucl. Phys. B B708, 434 (2005), hep-th/0 404064.

[25] H. Steinacker, J. High Energy Phys. 12, 049 (2007), arXiv: 0708.2426; H. Grosse, H. Steinacker and M. Wohlgenannt, ibid. 04, 023 (2008), arXiv:0802.0973; D. Klammer and H. Steinacker, ibid. 08, 074 (2008), arXiv:0805.1157; ibid. 02, 074 (2010), arXiv:0909.5298, H. Steinacker, Nucl. Phys. B 810, 1 (2009), arXiv: 0806.2032 J. High Energy Phys. 02, 044 (2009), arXiv:0812.3761

[26] D. Klammer and H. Steinacker, Phys. Rev. Lett. 102, 221301 (2009), arXiv:0903.0986.

[27] H. Steinacker, J. High Energy Phys. 12, 024 (2009), arXiv:0909.4621; D. N. Blaschke and H. Steinacker, Class. Quant. Grav. 27, 165010 (2010), arXiv:1003.4132,

[28] H. Steinacker, Class. Quant. Grav. 27, 133001 (2010), arXiv:1003.4134.

[29] A. D. Sakharov, Sov. Phys. Dokl. 12, 1040 (1968); A. H. Chamseddine and A. Connes, Commun. Math. Phys. 186, 731 (1997), hep-th/9606001; A. H. Chamseddine, A. Connes and M. Marcolli, Adv. Theor. Math. Phys. 11, 991 (2007), hep-th/0610241.

[30] J. Madore and J. Mourad, J. Math. Phys. 39, 423 (1998), gr-qc/9607060; S. I. Vacaru, Phys. Lett. B 498, 74 (2001), hep-th/ 0009163 , E. Langmann and R. J. Szabo, Phys. Rev. D 64, 104019 (2001), hep-th/0105094; R. J. Szabo, Gen. Relativ. Grav. 42, 1 (2010), arXiv:0906.2913.

[31] M. Burić, T. Grammatikopoulos, J. Madore and G. Zoupanos, J. High Energy Phys. 04, 054 (2006), hep-th/0603044, M. Burić, J. Madore and G. Zoupanos, SIGMA 3, 125 (2007), arXiv:0712.4024; M. Burić, H. Grosse and J. Madore, J. High Energy Phys. 07, 010 (2010), arXiv:1003.2284

[32] B. Muthukumar, Phys. Rev. D 71, 105007 (2005), hep-th/0412069; A. H. Fatollahi, Phys. Lett. B 665, 257 (2008), arXiv:0805.1159; A. Stern, Phys. Rev. D 80, 067703 (2009), arXiv:0907.4532, R. Banerjee, B. Chakraborty, S. Ghosh, P. Mukherjee and S. Samanta, Found. Phys. 39, 1297 (2009), arXiv:0909.1000. 
[33] H. Lin, O. Lunin and J. Maldacena, J. High Energy Phys. 10, 025 (2004), hep-th/0409174, H. Lin and J. Maldacena, Phys. Rev. D 74, 084014 (2006), hep-th/ 0509235.

[34] D. Berenstein, J. High Energy Phys. 01, 125 (2006), hep-th/0507203; D. Berenstein and D. H. Correa, ibid. 08, 006 (2006), hep-th/0511104; D. Berenstein and R. Cotta, Phys. Rev. D 74, 026006 (2006), hep-th/0605220; D. Berenstein, M. Hanada and S. A. Hartnoll, J. High Energy Phys. 02, 010 (2009), arXiv:0805.4658

[35] S. Iso, Y. Kimura, K. Tanaka and K. Wakatsuki, Nucl. Phys. B 604, 121 (2001), hep-th/0101102, R. Delgadillo-Blando, D. O'Connor and B. Ydri, Phys. Rev. Lett. 100, 201601 (2008), arXiv:0712.3011; J. High Energy Phys. 05, 049 (2009), arXiv:0806.0558, M. Hanada, Y. Hyakutake, J. Nishimura and S. Takeuchi, Phys. Rev. Lett. 102, 191602 (2009), arXiv:0811.3102, H. Aoki, J. Nishimura and Y. Susaki, J. High Energy Phys. 04, 055 (2009), arXiv:0810.5234, ibid. 09, 084 (2009), arXiv:0907.2107.

[36] T. Ishii, G. Ishiki, S. Shimasaki and A. Tsuchiya, Phys. Rev. D 78, 106001 (2008), arXiv:0807.2352, G. Ishiki, S.-W. Kim, J. Nishimura and A. Tsuchiya, J. High Energy Phys. 09, 029 (2009), arXiv:0907.1488.

[37] T. Ohl and A. Schenkel, Gen. Rel. Grav. 42, 2785 (2010), arXiv:0912.2252; A. Schenkel and C. F. Uhlemann, SIGMA 6, 061 (2010), arXiv:1003.3190.

[38] M. Salizzoni, A. Torrielli and H. S. Yang, Phys. Lett. B 634, 427 (2006), hep-th/0510249; H. S. Yang and M. Salizzoni, Phys. Rev. Lett. 96, 201602 (2006), hep-th/ 0512215.

[39] H. S. Yang, Mod. Phys. Lett. A 22, 1119 (2007), hep-th/0612231.

[40] H. S. Yang, Bulg. J. Phys. 35, 323 (2008), arXiv: 0711.0234.

[41] H. S. Yang, arXiv:0711.2797; Int. J. Mod. Phys. A 23, 2181 (2008), arXiv:0803.3272; Int. J. Mod. Phys. Conf. Ser. 1, 266 (2011), arXiv:0902.0035

[42] H. S. Yang and M. Sivakumar, Phys. Rev. D 82, 045004 (2010), arXiv:0908.2809,

[43] M. Kontsevich, Homological Algebra of Mirror Symmetry, alg-geom/ 9411018.

[44] G. Darboux, Sur le probl'eme de Pfaff, Bull. Sci. Math. 6, 14-36, 49-68 (1882).

[45] J. Moser, Trans. Amer. Math. Soc. 120, 286 (1965).

[46] F. J. Dyson, Am. J. Phys. 58, 209 (1990).

[47] C. R. Lee, Phys. Lett. A 148, 146 (1990).

[48] S. Tanimura, Ann. Phys. 220, 229 (1992), hep-th/ 9306066 ; J. F. Carinena, L. A. Ibort, G. Marmo and A. Stern, Phys. Rep. 263, 153 (1995), hep-th/9306066. 
[49] S. Sternberg, Proc. Natl. Acad. Sci. USA 74, 5253 (1977).

[50] M. Karoubi, K-Theory: An Introduction (Springer, Berlin, 1978).

[51] R. Minasian and G. Moore, J. High Energy Phys. 11, 002 (1997), hep-th/9710230; P. Hořava, Adv. Theor. Math. Phys. 2, 1373 (1998), hep-th/9812135.

[52] E. Witten, J. High Energy Phys. 12, 019 (1998), hep-th/9810188; Int. J. Mod. Phys. A 16, 063 (2001), hep-th/0007175.

[53] K. Olsen and R. J. Szabo, Adv. Theor. Math. Phys. 3, 889 (1999), hep-th/990714 0; J. A. Harvey, Komaba Lectures on Noncommutative Solitons and D-Branes, hep-th/ 0102076.

[54] M. F. Atiyah, R. Bott and A. Shapiro, Topology 3 (suppl. 1), 3 (1964).

[55] N. Hitchin, Quart. J. Math. Oxford Ser. 54, 281 (2003), math.DG/0209099; M. Gualtieri, math.DG/0401221; math.DG/0703298; G. R. Cavalcanti, math.DG/0501406.

[56] L. Cornalba, Adv. Theor. Math. Phys. 4, 271 (2000), hep-th/9909081.

[57] However, Eq. (56) shows that the $S U(2)$ gauge fields $A_{\mu}^{( \pm)}$have their own gauge fields, i.e., the vierbeins $E_{a}$ whose degrees of freedom match with those of the $U(1)$ gauge fields $D_{a}$ in the action in Eq. (44) when applying the map in Eq. (12). Therefore, it is sensible that $S U(2)$ gauge theory describing self-dual gravity can be mapped to the $U(1)$ gauge theory described by the action in Eq. (44), as will be shown later.

[58] S. W. Hawking, Phys. Lett. 60A, 81 (1977); T. Eguchi and A. J. Hanson, Phys. Lett. 74B, 249 (1978); G. W. Gibbons and S. W. Hawking, Phys. Lett. 78B, 430 (1978).

[59] J. J. Oh, C. Park and H. S. Yang, J. High Energy Phys. 04, 087 (2011), arXiv:1101.1357.

[60] Y. Hashimoto, Y. Yasui, S. Miyagi and T. Ootsuka, J. Math. Phys. 38, 5833 (1997), hep-th/9610069; T. Ootsuka, S. Miyagi, Y. Yasui and S. Zeze, Class. Quant. Grav. 16, 1305 (1999), gr-qc/9809083.

[61] One can show that the ansatz in Eq. (83) is equivalent to Eq. (69) (up to a sign) by the identification of the volume form $\nu=\lambda^{-2} E^{1} \wedge \cdots \wedge E^{4} \equiv \lambda^{-2} \nu_{g}$. See Eq. (95). According to the definition in Eq. (83), we have $J^{( \pm) i}=\frac{1}{2} \eta_{a b}^{( \pm) i} \iota_{a} \iota_{b}\left(\lambda^{-2} \nu_{g}\right)=\frac{1}{2} \eta_{a b}^{( \pm) i} \iota_{\lambda E_{a}} \iota_{\lambda E_{b}}\left(\lambda^{-2} \nu_{g}\right)=\frac{1}{2} \eta_{a b}^{( \pm) i} \iota_{E_{a}} \iota_{E_{b}} \nu_{g}=$ $\frac{1}{2}\left(\frac{1}{2} \varepsilon_{a b c d} \eta_{a b}^{( \pm) i}\right) E^{c} \wedge E^{d}= \pm \frac{1}{2} \eta_{a b}^{( \pm) i} E^{a} \wedge E^{b}$.

[62] A. Ashtekar, T. Jabobson and L. Smolin, Commun. Math. Phys. 115, 631 (1988); L. J. Mason and E. T. Newman, Commun. Math. Phys. 121, 659 (1989); S. Chakravarty, L. Mason and E. T. Newman, J. Math. Phys. 32, 1458 (1991); D. D. Joyce, Duke Math. J. 77, 519 (1995).

[63] This formula can be easily derived by applying Cartan's homotopy formula $\mathcal{L}_{X} \beta=\iota_{X} d \beta+d \iota_{X} \beta$ for 
$\beta=\iota_{Y} \alpha$ and $\left[\mathcal{L}_{X}, \iota_{Y}\right]=\iota_{[X, Y]}[8]$.

[64] N. Nekrasov and A. Schwarz, Commun. Math. Phys. 198, 689 (1998), hep-th/9802068; K.-Y. Kim, B.-H. Lee, H. S. Yang, J. Korean Phys. Soc. 41, 290 (2002), hep-th/ 00030 93; Phys. Lett. B 523, 357 (2001), hep-th/ 0109121.

[65] There is a perverse sign problem in reversing the map. We still don't understand how to properly translate the map $\Gamma(T M) \rightarrow C^{\infty}(M)$ to avoid the sign problem. In a way, one may consider some analytic continuation such that every module is defined over $\mathbb{C}$, as was suggested in Ref. [7]. Anyway, the sign issue will be insignificant at this stage.

[66] F. Bayen, M. Flato, C. Fronsdal, A. Lichnerowicz and D. Sternheimer, Ann. Phys. (N.Y.) 111, 61; 111 (1978); B. V. Fedosov, J. Diff. Geom. 40, 213 (1994).

[67] Here, $\hbar$ is a formal deformation parameter that may be identified with either $|\theta|$ or the Planck constant $\hbar$, depending on the choice of a Poisson manifold $M$ or $P$. For the Poisson manifold $M$ with the Poisson structure $\theta \in \Gamma\left(\Lambda^{2} T M\right)$, it would be convenient to take the rescaling $\theta^{\mu \nu} \rightarrow \hbar \theta^{\mu \nu}$ because $\hbar$ is regarded as a plain parameter without any indices. In the end, one may set $\hbar=1$.

[68] Our notation here may be sloppy. $D_{a}$ in Eq. (157) contains an extra $-i$ compared to Eq. (131). This sloppy notation is for a parallel march with $D_{\mu}$.

[69] R. S. Ward, Class. Quantum Grav. 7, L217 (1990).

[70] E. Corrigan, C. Devchand, D. B. Fairlie and J. Nuyts, Nucl. Phys. B 214, 452 (1983); R. S. Ward, Nucl. Phys. B 236, 381 (1984).

[71] D. Bak, K. Lee and J.-H. Park, Phys. Rev. D 66, 025021 (2002), hep-th/ 0204221.

[72] H. Elvang and J. Polchinski, C. R. Physique 4, 405 (2003), hep-th / 0209104.

[73] In this respect, quoting a recent comment by Zee [74] would be interesting: "The basic equation for the graviton field has the same form $g_{\mu \nu}=\eta_{\mu \nu}+h_{\mu \nu}$. This naturally suggests that $\eta_{\mu \nu}=\left\langle g_{\mu \nu}\right\rangle$ and perhaps some sort of spontaneous symmetry breaking." It turns out that this pattern is not an accidental happening.

[74] A. Zee, Int. J. Mod. Phys. A 23, 1295 (2008), arXiv: 0805.2183.

[75] L. Cornalba, J. High Energy Phys. 09, 017 (2000), hep-th/9912293; Adv. Theor. Math. Phys. 4, 1259 (2002), hep-th/ 0006018 ; L. Cornalba and R. Schiappa, Commun. Math. Phys. 225, 33 (2002), hep-th/0101219.

[76] Y. M. Cho and P. G. O. Freund, Phys. Rev. D 12, 1711 (1975).

[77] I. Vaisman, Lectures on the geometry of Poisson manifolds (Birkhäuser, Basel, 1994). 
[78] A. Weinstein, J. Diff. Geom. 18, 523 (1983).

[79] S. K. Wong, Nuovo Cimento 65A, 689 (1970).

[80] V. Guillemin and S. Sternberg, Symplectic Techniques in Physics (Cambridge University Press, 1984).

[81] A. Weinstein, Lett. Math. Phys. 2, 417 (1978).

[82] P. Hořava, Phys. Rev. Lett. 95, 016405 (2005), hep-th/ 0503006.

[83] G. E. Volovik, Lect. Notes Phys. 718, 31 (2007), cond-mat/0601372; Fermi-point scenario of emergent gravity, arXiv:0709.1258.

[84] H. Georgi, Lie Algebras in Particle Physics: From Isospin to Unified Theories (Advanced Book Program, 1999).

[85] One may imagine that the system in Eq. (277) is quantized a la Eq. (5). Then, the time evolution of a quantum system is derived from a Heisenberg algebra, i.e., $i \hbar \frac{d \widehat{f}}{d t}=[\widehat{f}, \widehat{H}]$ for $\widehat{f}, \widehat{H} \in \mathcal{A}_{\hbar}$. This is also in line with our philosophy that a geometry is derived from an algebra.

[86] H. S. Yang, J. Phys. Conf. Ser. 343, 012132 (2012), arXiv: 1111 . 0015.

[87] T. Padmanabhan, Gen. Rel. Grav. 40, 529 (2008), arXiv: 0705 . 2533; AIP Conf. Proc. 939, 114 (2007), arXiv:0706.1654; Adv. Sci. Lett. 2, 174 (2009), arXiv:0807.2356.

[88] This argument was corrected in Ref. [86], but we will not delete this incorrect argument to illustrate how a prejudice easily ignores important physics.

[89] S. Minwalla, M. Van Raamsdonk and N. Seiberg, J. High Energy Phys. 02, 020 (2000), hep-th/9912072.

[90] A. Raychaudhuri, Relativistic Cosmology. I, Phys. Rev. 98, 1123 (1955).

[91] S. W. Hawking and G. F. R. Ellis, The Large Scale Structure of Space-Time (Cambridge University Press, 1973).

[92] M. Blau and S. Theisen, Gen. Relativ. Grav. 41, 743 (2009). 